\title{
Finite dimensional boundary uniform stabilization of the Boussinesq system in Besov spaces by critical use of Carleman estimate-based inverse theory *
}

\author{
Irena Lasiecka ${ }^{\dagger} \quad$ Buddhika Priyasad ${ }^{\ddagger} \quad$ Roberto Triggiani ${ }^{\S}$
}

February 8, 2022

\begin{abstract}
We consider the d-dimensional Boussinesq system defined on a sufficiently smooth bounded domain, and subject to a pair $\{v, \boldsymbol{u}\}$ of controls localized on $\{\widetilde{\Gamma}, \omega\}$. Here, $v$ is a scalar Dirichlet boundary control for the thermal equation, acting on an arbitrary small connected portion $\widetilde{\Gamma}$ of the boundary $\Gamma=\partial \Omega$. Instead, $\boldsymbol{u}$ is a $d$-dimensional internal control for the fluid equation acting on an arbitrary small collar $\omega$ supported by $\widetilde{\Gamma}(\operatorname{Fig} 1)$. The initial conditions for both fluid and heat equations are taken of low regularity. We then seek to uniformly stabilize such Boussinesq system in the vicinity of an unstable equilibrium pair, in the critical setting of correspondingly low regularity spaces, by means of an explicitly constructed, finite dimensional feedback control pair $\{v, \boldsymbol{u}\}$ localized on $\{\widetilde{\Gamma}, \omega\}$. In addition, they will be minimal in number, and of reduced dimension: more precisely, $\boldsymbol{u}$ will be of dimension $(d-1)$, to include necessarily its $d^{\text {th }}$ component, and $v$ will be of dimension 1 . The resulting space of well-posedness and stabilization is a suitable, tight Besov space for the fluid velocity component (close to $\boldsymbol{L}^{3}(\Omega)$ for $d=3$ ) and a corresponding Besov space for the thermal component, $q>d$. Unique continuation inverse theorems for suitably over determined adjoint static problems play a critical role in the constructive solution. Their proof rests on Carleman-type estimates, a topic pioneered by M. V. Klibanov since the early 80's, after the 1939- breakthrough publication [17].
\end{abstract}

\section{Introduction.}

\subsection{Controlled dynamic Boussinesq equations.}

In this paper, we consider the following Boussinesq approximation equations in a bounded connected region $\Omega$ in $\mathbb{R}^{d}$ with sufficiently smooth boundary $\Gamma=\partial \Omega$. More specific requirements will be given below. Let $Q \equiv(0, T) \times \Omega$ and $\Sigma \equiv(0, T) \times \partial \Omega$ where $T>0$. Further, let $\omega$ be an arbitrary small open smooth subdomain of the region $\Omega, \omega \subset \Omega$, thus of positive measure which is a local collar supported by a corresponding connected arbitrary small portion $\widetilde{\Gamma}$ of the boundary $\Gamma=\partial \Omega$, Fig 1 .

${ }^{*}$ The research of I. L. and R. T. was partially supported by the National Science Foundation under Grant DMS-1713506. The research of B. P. was supported by the ERC advanced grant 668998 (OCLOC) under the EU's H2020 research program.

${ }^{\dagger}$ Department of Mathematical Sciences, The University of Memphis, Memphis, TN 38152, USA; IBS, Polish Academy of Sciences Warsaw, Poland

${ }^{\ddagger}$ Institute for Mathematics and Scientific Computing, University of Graz, Heinrichstrasse 36, A-8010 Graz, Austria. (b.sembukutti-liyanage@uni-graz.at).

${ }^{\S}$ Department of Mathematical Sciences, The University of Memphis, Memphis, TN 38152, USA. 


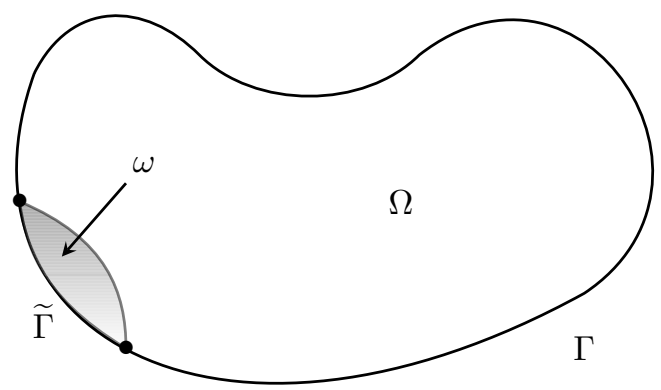

Fig 1

Let $m$ denote the characteristic function of $\omega: m(\omega) \equiv 1, m(\Omega / \omega) \equiv 0$.

Notation: Vector-valued functions and corresponding function spaces will be boldfaced. Thus, for instance, for the vector valued ( $d$-valued) velocity field or external force, we shall write say $\boldsymbol{y}, \boldsymbol{f} \in \boldsymbol{L}^{q}(\Omega)$ rather than $y, f \in\left(L^{q}(\Omega)\right)^{d}$.

We consider the Boussinesq system under the action of a control pair $\{v, \boldsymbol{u}\}$ localized on $\{\widetilde{\Gamma}, \omega\}$. Here $v$ is a scalar Dirichlet boundary control for the thermal equation acting on $\widetilde{\Gamma}$, while $\boldsymbol{u}$ is a $d$-dimensional vector interior control acting as $m(x) \boldsymbol{u}(t, x)$ on $\omega$ :

$$
\left\{\begin{aligned}
\boldsymbol{y}_{t}-\nu \Delta \boldsymbol{y}+(\boldsymbol{y} \cdot \nabla) \boldsymbol{y}-\gamma(\theta-\bar{\theta}) \boldsymbol{e}_{d}+\nabla \pi & =m(x) \boldsymbol{u}(t, x)+\boldsymbol{f}(x) \text { in } Q \\
\theta_{t}-\kappa \Delta \theta+\boldsymbol{y} \cdot \nabla \theta & =g(x) \text { in } Q \\
\operatorname{div} \boldsymbol{y} & =0 \text { in } Q \\
\boldsymbol{y}=0, \theta & =v \text { on } \Sigma \\
\boldsymbol{y}(0, x)=\boldsymbol{y}_{0}, \quad \theta(0, x) & =\theta_{0} \text { on } \Omega .
\end{aligned}\right.
$$

In the Boussinesq approximation system, $\boldsymbol{y}=\left\{y_{1}, \ldots, y_{d}\right\}$ represents the fluid velocity, $\theta$ the scalar temperature of the fluid, $\nu$ the kinematic viscosity coefficient, $\kappa$ the thermal conductivity. The scalar function $\pi$ is the unknown pressure. The term $\boldsymbol{e}_{d}$ denotes the vector $(0, \ldots, 0,1)$. Moreover $\gamma=\bar{g} / \bar{\theta}$ where $\bar{g}$ is the acceleration due to gravity and $\bar{\theta}$ is the reference temperature. The $d$-vector valued function $\boldsymbol{f}(x)$ and scalar function $g(x)$ correspond to an external force acting on the Navier-Stokes equations and a heat source density acting on the heat equation, respectively. They are given along with the I.C.s $\boldsymbol{y}_{0}$ and $\theta_{0}$, which are assumed of low regularity. Note that $\boldsymbol{y} \cdot \nabla \theta=\operatorname{div}(\theta \boldsymbol{y})$.

The Boussinesq system models heat transfer in a viscous incompressible heat conducting fluid. It consists of the Navier-Stokes equations (in the vector velocity $\boldsymbol{y}$ ) 83. coupled with the convection-diffusion equation (for the scalar temperature $\theta$ ). The external body force $\boldsymbol{f}(x)$ and the heat source density $g(x)$ may render the overall system unstable in the technical sense described below by (1.38). The goal of the paper is to exploit the localized controls $\boldsymbol{u}$ on $\omega$ and $v$ on $\widetilde{\Gamma}$, sought to be finite dimensional and in feedback form, in order to stabilize the overall system. Their minimal number will be equal to the maximal geometric multiplicity of the unstable eigenvalues in (1.38). As an additional benefit of our investigation, the feedback fluid component of $\boldsymbol{u}$ will be of reduced dimension $(d-1)$ rather than $d$, to include necessarily the $d^{\text {th }}$ component of $\boldsymbol{u}$, while the feedback heat component of $v$ will be 1-dimensional. This is a consequence of the Unique Continuation Inverse Theory type of property expressed by Theorem B.1. Appendix B for the adjoint static problem. Its proof [89] after [86] rests on Carleman-type estimates, a topic consistently pursued by M. V. Klibanov since his pioneering work in the early 80's, 
after the breakthrough publication [17. This theme will be further explored in Section 2.5, For now, we note that a Unique Continuation Property is not only a critical theoretical tool. It is also fundamental in computations, where it is realized numerically, as in recent work of M. V. Klibanov and his team (See papers [42] and [43] and references cited therein) on coefficient inverse problems. Here use is made of a globally convergent numerical method, referred as convexification principle, to solve numerically boundary value problems with Cauchy boundary data for coupled systems of quasilinear elliptic equations. To this end, the convexification constructs a weighted globally strict convex Tikhonov-like functional, which involves a Carleman-type weight. As far as practical applications of the Boussinesq model are concerned, one may consider the situation of controlling the temperature and humidity in a bounded restricted environment - see [15], 16] for an eloquent description of the physical phenomenon. Due to the physical significance of the Boussinesq system, the problem of its stabilization has been considered in the literature - with both localized and boundary controls - following of course prior developments concerning the Navier-Stokes model alone. See subsection 2.5 for a review of the literature.

\section{Motivation: why studying uniform stabilization of the Boussinesq problem (1.1) in the Besov functional setting of the present paper?}

In short: Stimulated by recent research achievements [60, 62] on the uniform stabilization of the Navier-Stokes equations - to be elaborated below in Section [2.5]- the present paper sets the stage as a preliminary, needed step toward the authors' final goal of solving the Boussinesq uniform stabilization problem with a set of three localized controls $\left\{v_{h}, \boldsymbol{v}_{f}, \boldsymbol{u}\right\}$ all finite dimensional, in feedback form and of minimal number: a scalar control $v_{h}$ acting on heat $\theta$-component as in interior control localized on the small set $\omega$, and a pair $\left\{\boldsymbol{v}_{f}, \boldsymbol{u}\right\}$ of controls acting on the Navier-Stokes fluid $\boldsymbol{y}$-component, as in 62] : that is, $\boldsymbol{v}_{f}$ as a boundary tangential control acting on $\widetilde{\Gamma}$ and $\boldsymbol{u}$ as an interior tangential-like control acting on $\omega$. See Fig 1. To obtain finite dimensionality of $\boldsymbol{v}_{f}$ for $d=3$, it is critical to seek uniform stabilization on the tight Besov setting of the present paper, which for $d=3$ is "close" to the space $\boldsymbol{L}^{3}$, well-known [24, [39, [72] to be a critical space for the well-posedness of the uncontrolled 3-d Navier-Stokes equation in $\mathbb{R}^{3}$. Thus, the present paper serves also as a testing ground toward such final goal.

\subsection{Stationary Boussinesq equations.}

Our starting point is the following result.

Theorem 1.1. Consider the following steady-state Boussinesq system in $\Omega$

$$
\left\{\begin{array}{rlrl}
-\nu \Delta \boldsymbol{y}_{e}+\left(\boldsymbol{y}_{e} \cdot \nabla\right) \boldsymbol{y}_{e}-\gamma\left(\theta_{e}-\bar{\theta}\right) \boldsymbol{e}_{d}+\nabla \pi_{e} & =\boldsymbol{f}(x) & & \text { in } \Omega \\
-\kappa \Delta \theta_{e}+\boldsymbol{y}_{e} \cdot \nabla \theta_{e} & =g(x) & \text { in } \Omega \\
\operatorname{div} \boldsymbol{y}_{e} & =0 & & \text { in } \Omega \\
\boldsymbol{y}_{e}=0, \theta_{e} & =0 & & \text { on } \partial \Omega .
\end{array}\right.
$$

Let $1<q<\infty$. For any $\boldsymbol{f}, g \in \boldsymbol{L}^{q}(\Omega), L^{q}(\Omega)$, there exists a solution (generally not unique) $\left(\boldsymbol{y}_{e}, \theta_{e}, \pi_{e}\right) \in$ $\left(\boldsymbol{W}^{2, q}(\Omega) \cap \boldsymbol{W}_{0}^{1, q}(\Omega)\right) \times\left(W^{2, q}(\Omega) \cap W_{0}^{1, q}(\Omega)\right) \times\left(W^{1, q}(\Omega) / \mathbb{R}\right)$.

See [3, 44, [5] for $q \neq 2$. In the Hilbert space setting, see [19], [27], [74], [90], 44].

Remark 1.1. (i) Let the given external force $\boldsymbol{f}$ be conservative, $\boldsymbol{f}=\nabla \phi$ and the heat source density $g \equiv 0$. Then, $\boldsymbol{y}_{e} \equiv 0, \theta_{e} \equiv 0, \nabla \pi_{e}=\nabla f-\bar{g} \boldsymbol{e}_{d}$ is a solution of problem (1.2a-d).

(ii) In the case of equilibrium solution $\boldsymbol{y}_{e}$ for just the Navier-Stokes equation, it is well-known [55, 65, 83. that the stationary solution is unique when "the data is small enough, or the viscosity is large enough" [83, p 157; Chapt 2] that is, if the ratio $\|f\| / \nu_{o}^{2}$ is smaller than some constant that depends only on $\Omega$ [27, p 121]. When non-uniqueness occurs, the stationary solutions depend on a finite number of parameters [27, Theorem 2.1, p 121] asymptotically, in the time dependent case. 
(iii) In this paper, we take one equilibrium solution $\left\{\boldsymbol{y}_{e}, \theta_{e}\right\}$, when non-unique and make the subsequent analysis related to such choice.

\subsection{A first quantitative description of the main goal of the present paper.}

The starting point of the present paper is the following: that under a given external force $\boldsymbol{f}(x)$ for the fluid equations, a given heat source $g(x)$ for the thermal equation, and given viscosity coefficient $\nu$ and thermal conductivity $\kappa$, the equilibrium solution $\left\{\boldsymbol{y}_{e}, \theta_{e}\right\}$ is unstable, in a quantitative sense to be made more precise in sub-section [1.7, specifically in (1.38). This will mean that the free dynamics linear operator $\mathbb{A}_{q}$ defined in (1.34) - which has compact resolvent, and is the generator of a s.c. analytic semigroup in the appropriate functional setting (Theorem [1.2) - has $N$ unstable eigenvalues.

The main goal of the present paper is then - at first qualitatively - to feedback stabilize the non-linear Boussinesq model (1.1) subject to rough (non-smooth) initial conditions $\left\{\boldsymbol{y}_{0}, \theta_{0}\right\}$, in the vicinity of an (unstable) equilibrium solution $\left\{\boldsymbol{y}_{e}, \theta_{e}\right\}$ in (1.2), by means of a finite dimensional localized feedback control pair $\{v, m \boldsymbol{u}\}$ as acting on $\{\widetilde{\Gamma}, \omega\}$, see Fig 1. Thus this paper pertains to the general issue of "turbulence suppression or attenuation" in fluids. The general topic of turbulence suppression (or attenuation) in fluids has been the object of many studies over the years, mostly in the engineering literature - through experimental studies and via numerical simulation - and under different geometrical and dynamical settings. The references cited in the present paper by necessity pertain mostly to the mathematical literature. A more precise description of our paper is as follows: establish localized exponential stabilization of problem (1.1) near an unstable equilibrium solution $\left\{\boldsymbol{y}_{e}, \theta_{e}\right\}$ by means of a finite dimensional localized, spectral-based feedback control pair $\{v, m \boldsymbol{u}\}$, in the important case of initial condition $\boldsymbol{y}_{0}$ of low regularity, as technically expressed by $\boldsymbol{y}_{0}$ being in a suitable Besov space with tight indices, and $\theta_{0}$ being in a corresponding $L^{q}$-space $q>d$, or even in a corresponding Besov space, see Remark 1.3. More precisely, the resulting state space for the pair $\{\boldsymbol{y}, \theta\}$, where uniform stabilization will be achieved is the space

$$
\boldsymbol{V}^{q, p}(\Omega) \equiv \widetilde{\boldsymbol{B}}_{q, p}^{2-2 / p}(\Omega) \times L^{q}(\Omega), 1<p<\frac{2 q}{2 q-1} ; q>d, d=2,3,
$$

or even

$$
\boldsymbol{V}_{b}^{q, p}(\Omega) \equiv \widetilde{\boldsymbol{B}}_{q, p}^{2-2 / p}(\Omega) \times B_{q, p}^{2-2 / p}(\Omega), 1<p<\frac{2 q}{2 q-1} ; q>d, d=2,3,
$$

where $\widetilde{\boldsymbol{B}}_{q, p}^{2-2 / p}(\Omega)$ is a suitable subspace, see below in (1.11), of the Besov space

$$
\left(\boldsymbol{L}^{q}(\Omega), \boldsymbol{W}^{2, q}(\Omega)\right)_{1-1 / p, p}=\boldsymbol{B}_{q, p}^{22^{2} / p}(\Omega), \quad 1<p<\frac{2 q}{2 q-1} ; q>d, d=2,3,
$$

as a real interpolation space between $\boldsymbol{L}^{q}(\Omega)$ and $\boldsymbol{W}^{2, q}(\Omega)$. Similarly for $B_{q, p}^{2-^{2} / p}(\Omega)$. This setting will be further elaborated after introducing the Helmholtz decomposition below. In particular, local exponential stability for the velocity field $\boldsymbol{y}$ near an equilibrium solution $\boldsymbol{y}_{e}$ will be achieved in the topology of the Besov subspace $\widetilde{\boldsymbol{B}}_{q, p}^{2-1 / p}(\Omega)$ in (1.11). Note the tight index: $1<p<6 / 5$ for $q>d=3$, and $1<p<4 / 3$ for $d=2$. For $d=3$, such space is "close" to $\boldsymbol{L}^{3}(\Omega)$. It will be documented below in Remark 1.2 that in such a setting, the compatibility conditions on the boundary of the initial conditions are not recognized. This feature is precisely our key objective within the stabilization problem of the 3-dimensional NavierStokes equations per se [62], or for the Boussinesq system with controls $\left\{\boldsymbol{v}_{f}, \boldsymbol{u}\right\}$ on the N-S component, as described in the "motivation" paragraph at the end of Section 1.1 .

\subsection{Helmholtz decomposition.}

A first difficulty one faces in extending the local exponential stabilization results for fluids such as the Navier-Stokes equations or Boussinesq systems from the Hilbert-space setting as in [64, [92] to the 
$\boldsymbol{L}^{q}$-based setting is the question of the existence of a Helmholtz (Leray) projection for the domain $\Omega$ in $\mathbb{R}^{d}$. More precisely: Given an open set $\Omega \subset \mathbb{R}^{d}$, the Helmholtz decomposition answers the question as to whether $\boldsymbol{L}^{q}(\Omega)$ can be decomposed into a direct sum of the solenoidal vector space $\boldsymbol{L}_{\sigma}^{q}(\Omega)$ and the space $\boldsymbol{G}^{q}(\Omega)$ of gradient fields. Here,

$$
\begin{aligned}
& \boldsymbol{L}_{\sigma}^{q}(\Omega)={\overline{\left\{\boldsymbol{y} \in \boldsymbol{C}_{c}^{\infty}(\Omega): \operatorname{div} \boldsymbol{y}=0 \text { in } \Omega\right\}}}^{\|\cdot\|_{q}} \\
& =\left\{\boldsymbol{g} \in \boldsymbol{L}^{q}(\Omega): \operatorname{div} \boldsymbol{g}=0 ; \boldsymbol{g} \cdot \nu=0 \text { on } \partial \Omega\right\}, \\
& \text { for any locally Lipschitz domain } \Omega \subset \mathbb{R}^{d}, d \geq 2 \\
& \boldsymbol{G}^{q}(\Omega)=\left\{\boldsymbol{y} \in \boldsymbol{L}^{q}(\Omega): \boldsymbol{y}=\nabla p, p \in W_{l o c}^{1, q}(\Omega)\right\} \text { where } 1 \leq q<\infty .
\end{aligned}
$$

Both of these are closed subspaces of $\boldsymbol{L}^{q}$.

Definition 1.1. Let $1<q<\infty$ and $\Omega \subset \mathbb{R}^{n}$ be an open set. We say that the Helmholtz decomposition for $\boldsymbol{L}^{q}(\Omega)$ exists whenever $\boldsymbol{L}^{q}(\Omega)$ can be decomposed into the direct sum (non-orthogonal for $q \neq 2$, orthogonal for $q=2$ )

$$
\boldsymbol{L}^{q}(\Omega)=\boldsymbol{L}_{\sigma}^{q}(\Omega) \oplus \boldsymbol{G}^{q}(\Omega) .
$$

The unique linear, bounded and idempotent (i.e. $P_{q}^{2}=P_{q}$ ) projection operator $P_{q}: \boldsymbol{L}^{q}(\Omega) \longrightarrow \boldsymbol{L}_{\sigma}^{q}(\Omega)$ having $\boldsymbol{L}_{\sigma}^{q}(\Omega)$ as its range and $\boldsymbol{G}^{q}(\Omega)$ as its null space is called the Helmholtz projection. For additional information we refer to [30], [61, Appendix B] and references therein. In particular, throughout the paper we shall use freely that $\left(\boldsymbol{L}_{\sigma}^{q}(\Omega)\right)^{\prime}=\boldsymbol{L}_{\sigma}^{q^{\prime}}(\Omega),{ }^{1} / q+{ }^{1} / q^{\prime}=1$.

While for $q=2$ a Helmholtz decomposition (in fact orthogonal decomposition) exists for any open set $\Omega \subset \mathbb{R}^{d}$, this is not the case for $q \neq 2\left[67\right.$. However, for a bounded convex domain $\Omega \subset \mathbb{R}^{d}, d \geq 2,1<$ $q<\infty$ [25] or for a bounded $C^{1}$-domain in $\mathbb{R}^{d}[25$, the Helmholtz decomposition is known to be true. This is the case in the present paper. We can now provide further critical information on the Besov space $\widetilde{\boldsymbol{B}}_{q, p}^{2-2 / p}(\Omega)$ which is the fluid component of the state space (1.3) where well-posedness and uniform stabilization take place for the resulting closed-loop feedback problem.

Definition of Besov spaces $\boldsymbol{B}_{q, p}^{s}(\Omega)$ on domains of class $C^{1}$ as real interpolation of Sobolev spaces: Let $m$ be a positive integer, $m \in \mathbb{N}, 0<s<m, 1 \leq q<\infty, 1 \leq p \leq \infty$, then we define the Besov space [1]

$$
\boldsymbol{B}_{q, p}^{s}(\Omega)=\left(\boldsymbol{L}^{q}(\Omega), \boldsymbol{W}^{m, q}(\Omega)\right)_{\frac{s}{m}, p}
$$

as a real interpolation space between $\boldsymbol{L}^{q}(\Omega)$ and $\boldsymbol{W}^{m, q}(\Omega)$. This definition does not depend on $m \in \mathbb{N}$ [11, $\mathrm{p} \mathrm{xx}$. This clearly gives

$$
\boldsymbol{W}^{m, q}(\Omega) \subset \boldsymbol{B}_{q, p}^{s}(\Omega) \subset \boldsymbol{L}^{q}(\Omega) \quad \text { and } \quad\|\boldsymbol{y}\|_{\boldsymbol{L}^{q}(\Omega)} \leq C\|\boldsymbol{y}\|_{\boldsymbol{B}_{q, p}^{s}(\Omega)} .
$$

We shall be particularly interested in the following special real interpolation space of the $\boldsymbol{L}^{q}$ and $\boldsymbol{W}^{2, q}$ spaces $\left(m=2, s=2-\frac{2}{p}\right)$ :

$$
\boldsymbol{B}_{q, p}^{2-\frac{2}{p}}(\Omega)=\left(\boldsymbol{L}^{q}(\Omega), \boldsymbol{W}^{2, q}(\Omega)\right)_{1-\frac{1}{p}, p}
$$

Our interest in (1.9) is due to the following characterization [2, Thm 3.4]: if $A_{q}$ denotes the Stokes operator to be introduced in (1.14) below, then

$$
\begin{array}{r}
\left(\boldsymbol{L}_{\sigma}^{q}(\Omega), \mathcal{D}\left(A_{q}\right)\right)_{1-\frac{1}{p}, p}=\left\{\boldsymbol{g} \in \boldsymbol{B}_{q, p}^{2-{ }^{2} / p}(\Omega): \operatorname{div} \boldsymbol{g}=0,\left.\boldsymbol{g}\right|_{\Gamma}=0\right\} \\
\text { if } \frac{1}{q}<2-\frac{2}{p}<2
\end{array}
$$




$$
\begin{gathered}
\left(\boldsymbol{L}_{\sigma}^{q}(\Omega), \mathcal{D}\left(A_{q}\right)\right)_{1-\frac{1}{p}, p}=\left\{\boldsymbol{g} \in \boldsymbol{B}_{q, p}^{22^{2 / p}}(\Omega): \operatorname{div} \boldsymbol{g}=0,\left.\boldsymbol{g} \cdot \nu\right|_{\Gamma}=0\right\} \\
\equiv \widetilde{\boldsymbol{B}}_{q, p}^{2-2 / p}(\Omega) \text { if } 0<2-\frac{2}{p}<\frac{1}{q} ; \text { or } 1<p<\frac{2 q}{2 q-1} .
\end{gathered}
$$

Remark 1.2. Notice that, in (1.11), the condition $\left.\boldsymbol{g} \cdot \nu\right|_{\Gamma}=0$ is an intrinsic condition of the space $\boldsymbol{L}_{\sigma}^{q}(\Omega)$ in (1.3), not an extra boundary condition as $\left.\boldsymbol{g}\right|_{\Gamma}=0$ in (1.10).

Orientation: As already noted,ultimately, we shall seek to obtain uniform feedback stabilization of the fluid component $\boldsymbol{y}$ in the Besov subspace $\widetilde{\boldsymbol{B}}_{q, p}^{2-{ }^{2 / p}}(\Omega), \operatorname{dim} \Omega=d<q<\infty, 1<p<{ }^{2 q} / 2 q-1$, defined by real interpolation in (1.4), (1.11); The reason being that such a space does not recognize boundary conditions, as noted above in Remark 1.2. Analyticity and maximal regularity of the Stokes problem will require $q>1$, Appendix $\mathrm{A}$.

By way of orientation, we state at the outset two main points. For the linearized $\boldsymbol{w}$-problem (1.31) or (1.32) below in the feedback form (2.4) or (5.3), the corresponding well-posedness and global feedback uniform stabilization result, Theorem [2.1] or Theorems 6.1] and 6.2, hold in general for $1<q<\infty$. Instead, the final, main well-posedness and feedback uniform, local stabilization results, Theorems 2.2 and 2.3, the latter for the nonlinear feedback problem (2.11) or (8.3) corresponding to the original problem (1.1) via its translation (1.13), will require $q>3$ to obtain the embedding $\boldsymbol{W}^{1, q}(\Omega) \hookrightarrow \boldsymbol{L}^{\infty}(\Omega)$ in our case of interest $d=3$, see (8.24), hence $1<p<6 / 5$; and $q>2$, hence $1<p<4 / 3$ in the $d=2$-case. The ultimate main result for the original problem (1.1) is Theorem [2.4.

\subsection{Translated nonlinear Boussinesq problem and its abstract model.}

PDE Model: We return to Theorem 1.1 which provides an equilibrium triplet $\left\{\boldsymbol{y}_{e}, \theta_{e}, \pi_{e}\right\}$. Then, we translate by $\left\{\boldsymbol{y}_{e}, \theta_{e}, \pi_{e}\right\}$ the original Boussinesq problem (1.1). Thus we introduce new variables

$$
\boldsymbol{z}=\boldsymbol{y}-\boldsymbol{y}_{e} \quad \text { (a } d \text {-vector) }, \quad h=\theta-\theta_{e} \quad(\text { a scalar }), \quad \chi=\pi-\pi_{e} \quad \text { (a scalar) }
$$

and obtain the translated problem

$$
\left\{\begin{array}{r}
f_{t}-\nu \Delta \boldsymbol{z}+\left(\boldsymbol{y}_{e} \cdot \nabla\right) \boldsymbol{z}+(\boldsymbol{z} \cdot \nabla) \boldsymbol{y}_{e}+(\boldsymbol{z} \cdot \nabla) \boldsymbol{z}-\gamma h \boldsymbol{e}_{d}+\nabla \chi \\
h_{t}-\kappa \Delta h+\boldsymbol{y}_{e} \cdot \nabla h+\boldsymbol{u} \cdot \nabla h+\boldsymbol{z} \cdot \nabla \theta_{e}=0 \text { in } Q \\
\operatorname{div} \boldsymbol{z}=0 \text { in } Q \\
\boldsymbol{z}=0, h=v \text { on } \Sigma \\
\boldsymbol{z}(0, x)=\boldsymbol{z}_{0}=\boldsymbol{y}_{0}-\boldsymbol{y}_{e}, \quad h(0, x)=h_{0}=\theta-\theta_{e} \text { on } \Omega \\
L_{e}(\boldsymbol{z})=\left(\boldsymbol{y}_{e} \cdot \nabla\right) \boldsymbol{z}+(\boldsymbol{z} \cdot \nabla) \boldsymbol{y}_{e} \quad \text { (Oseen perturbation). }
\end{array}\right.
$$

Abstract Nonlinear Translated Model. First, for $1<q<\infty$ fixed, the Stokes operator $A_{q}$ in $\boldsymbol{L}_{\sigma}^{q}(\Omega)$ with Dirichlet boundary conditions is defined by

$$
A_{q} \boldsymbol{z}=-P_{q} \Delta \boldsymbol{z}, \quad \mathcal{D}\left(A_{q}\right)=\boldsymbol{W}^{2, q}(\Omega) \cap \boldsymbol{W}_{0}^{1, q}(\Omega) \cap \boldsymbol{L}_{\sigma}^{q}(\Omega) .
$$

The operator $A_{q}$ has a compact inverse $A_{q}^{-1}$ on $\boldsymbol{L}_{\sigma}^{q}(\Omega)$, hence $A_{q}$ has a compact resolvent on $\boldsymbol{L}_{\sigma}^{q}(\Omega)$. Its properties are collected in Appendix [A. Next, we introduce the first order operator $A_{o, q}$,

$$
A_{o, q} \boldsymbol{z}=P_{q}\left[\left(\boldsymbol{y}_{e} \cdot \nabla\right) \boldsymbol{z}+(\boldsymbol{z} \cdot \nabla) \boldsymbol{y}_{e}\right], \quad \mathcal{D}\left(A_{o, q}\right)=\mathcal{D}\left(A_{q}^{1 / 2}\right) \subset \boldsymbol{L}_{\sigma}^{q}(\Omega),
$$

where the $\mathcal{D}\left(A_{q}^{1 / 2}\right)$ is defined explicitly by complex interpolation

$$
\left[\mathcal{D}\left(A_{q}\right), \boldsymbol{L}_{\sigma}^{q}(\Omega)\right]_{\frac{1}{2}}=\mathcal{D}\left(A_{q}^{1 / 2}\right) \equiv \boldsymbol{W}_{0}^{1, q}(\Omega) \cap \boldsymbol{L}_{\sigma}^{q}(\Omega) .
$$


Thus, $A_{o, q} A_{q}^{-1 / 2}$ is a bounded operator on $\boldsymbol{L}_{\sigma}^{q}(\Omega)$, and thus $A_{o, q}$ is bounded on $\mathcal{D}\left(A_{q}^{1 / 2}\right)$

$$
\left\|A_{o, q} f\right\|=\left\|A_{o, q} A_{q}^{-1 / 2} A_{q}^{-1 / 2} A_{q} f\right\| \leq C_{q}\left\|A_{q}^{1 / 2} f\right\|, \quad f \in \mathcal{D}\left(A_{q}^{1 / 2}\right) .
$$

This leads to the definition of the Oseen operator for the fluid

$$
\mathcal{A}_{q}=-\left(\nu A_{q}+A_{o, q}\right), \quad \mathcal{D}\left(\mathcal{A}_{q}\right)=\mathcal{D}\left(A_{q}\right) \subset \boldsymbol{L}_{\sigma}^{q}(\Omega) .
$$

We next define the differential operator of the heat component in $1.13 \mathrm{~b}$

$$
\mathcal{B}_{q} f=-\kappa \Delta f+\boldsymbol{y}_{e} \cdot \nabla f, \quad \mathcal{D}\left(\mathcal{B}_{q}\right)=W^{2, q}(\Omega) \cap W_{0}^{1, q}(\Omega) .
$$

Then, we define the projection of the nonlinear portion of the fluid operator in (1.13a)

$$
\mathcal{N}_{q}(\boldsymbol{z})=P_{q}[(\boldsymbol{z} \cdot \nabla) \boldsymbol{z}], \quad \mathcal{D}\left(\mathcal{N}_{q}\right)=\boldsymbol{W}^{1, q}(\Omega) \cap \boldsymbol{L}^{\infty}(\Omega) \cap \boldsymbol{L}_{\sigma}^{q}(\Omega) .
$$

(Recall that $W^{1, q}(\Omega) \hookrightarrow L^{\infty}(\Omega)$ for $q>d=\operatorname{dim} \Omega$ [41, p. 74]). Next, we define the nonlinear coupled term of the heat equation as

$$
\mathcal{M}_{q}[\boldsymbol{z}](h)=\boldsymbol{z} \cdot \nabla h, \quad \mathcal{D}\left(\mathcal{M}_{q}[\boldsymbol{z}]\right)=W^{1, q}(\Omega) \cap L^{\infty}(\Omega) .
$$

Finally, we define the coupling linear terms as bounded operators on $L^{q}(\Omega), \boldsymbol{L}_{\sigma}^{q}(\Omega)$ respectively, $q>d$ :

$$
\begin{gathered}
\text { [from the NS equation] } \mathcal{C}_{\gamma} h=-\gamma P_{q}\left(h \boldsymbol{e}_{d}\right), \mathcal{C}_{\gamma} \in \mathcal{L}\left(L^{q}(\Omega), \boldsymbol{L}_{\sigma}^{q}(\Omega)\right), \\
\text { [from the heat equation] } \mathcal{C}_{\theta_{e}} \boldsymbol{z}=\boldsymbol{z} \cdot \nabla \theta_{e}, \mathcal{C}_{\theta_{e}} \in \mathcal{L}\left(\boldsymbol{L}_{\sigma}^{q}(\Omega), L^{q}(\Omega)\right) .
\end{gathered}
$$

Next, in preparation to the abstract version of the non-linear $\boldsymbol{z}$-system (1.13), we introduce the Dirichlet map $D$ [58] with reference to the Dirichlet boundary controlled thermal equation (1.13b) in $h$ :

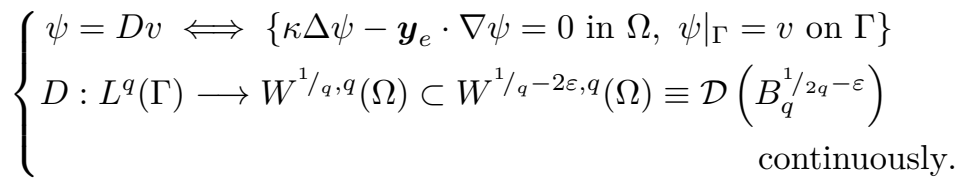

[91, Theorem III.2.3, p91] where $B_{q}$ is the Dirichlet Laplacian on $L^{q}(\Omega)$

$$
B_{q} f=-\Delta f, \quad \mathcal{D}\left(B_{q}\right)=\mathcal{D}\left(\mathcal{B}_{q}\right)=W^{2, q}(\Omega) \cap W_{0}^{1, q}(\Omega) .
$$

Accordingly, we rewrite Eq (1.13b) via (1.18), (1.23)

$$
h_{t}-\left(\kappa \Delta-\boldsymbol{y}_{e} \cdot \nabla\right)(h-D v)+\boldsymbol{z} \cdot \nabla h+\boldsymbol{z} \cdot \nabla \theta_{e}=0 \text { in } Q
$$

where $[h-D v]_{\Gamma}=0$ by (1.13d) and (1.23a). Accordingly, invoking the operators $\mathcal{B}_{q}$ from (1.18) as well as $\mathcal{M}_{q}[\boldsymbol{z}], \mathcal{C}_{\theta_{e}}$ from (1.20), (1.22) respectively, we can rewrite Eq (1.24) abstractly as

$$
h_{t}+\mathcal{B}_{q}(h-D v)+\mathcal{M}_{q}[\boldsymbol{z}] h+\mathcal{C}_{\theta_{e}} \boldsymbol{z}=0 .
$$

Extending as usual [58, the original operator $\mathcal{B}_{q}: L^{q}(\Omega) \supset \mathcal{D}\left(\mathcal{B}_{q}\right) \longrightarrow L^{q}(\Omega)$ into $\left(L^{q}(\Omega)\right)^{\prime}=L^{q^{\prime}}(\Omega) \longrightarrow$ $\left[\mathcal{D}\left(\mathcal{B}_{q}^{*}\right)\right]^{\prime}$ (duality w.r.t. $L^{q}$ ) and retaining the same symbol $\mathcal{B}_{q}$ for the extension, we see that we can rewrite Eq (1.25) as

$$
h_{t}+\mathcal{B}_{q} h+\mathcal{M}_{q}[\boldsymbol{z}] h+\mathcal{C}_{\theta_{e}} \boldsymbol{z}=\mathcal{B}_{q} D v \in\left[\mathcal{D}\left(B_{q}^{*}\right)\right]^{\prime} .
$$

Next we apply the Helmholtz projector $P_{q}$ on the coupled N-S equation (1.13a), invoke the operators introduced above - specifically, $\mathcal{A}_{q}$ from (1.17), $\mathcal{N}_{q}$ from (1.19), and $\mathcal{C}_{\gamma}$ from (1.21)- and obtain the following abstract version of the controlled fluid equation

$$
\boldsymbol{z}_{t}-\mathcal{A}_{q} \boldsymbol{z}+\mathcal{N}_{q} \boldsymbol{z}+\mathcal{C}_{\gamma} h=P_{q}(m \boldsymbol{u}) \text { in } \boldsymbol{L}_{\sigma}^{q}(\Omega)
$$


In conclusion, combining Eq (1.27) with Eq (1.26) we obtain the abstract version of the original boundary controlled Boussinesq system (1.13a-e)

$$
\left\{\begin{array}{rlrl}
\boldsymbol{z}_{t}-\mathcal{A}_{q} \boldsymbol{z}+\mathcal{N}_{q} \boldsymbol{z}+\mathcal{C}_{\gamma} h & =P_{q}(m \boldsymbol{u}) & \text { in } \boldsymbol{L}_{\sigma}^{q}(\Omega) \\
h_{t}+\mathcal{B}_{q} h+\mathcal{M}_{q}[\boldsymbol{z}] h+\mathcal{C}_{\theta_{e}} \boldsymbol{z} & =\mathcal{B}_{q} D v & \text { in }\left[\mathcal{D}\left(B_{q}^{*}\right)\right]^{\prime} \subset L^{q^{\prime}}(\Omega) \\
\boldsymbol{z}(x, 0) & =\boldsymbol{z}_{0}(x) & & \text { in } \boldsymbol{L}_{\sigma}^{q}(\Omega) \\
h(x, 0) & =h_{0}(x) & & \text { in } L^{q}(\Omega) ;
\end{array}\right.
$$

or in matrix form

$$
\left\{\begin{array}{l}
\frac{d}{d t}\left[\begin{array}{l}
\boldsymbol{z} \\
h
\end{array}\right]=\left[\begin{array}{cc}
\mathcal{A}_{q} & -\mathcal{C}_{\gamma} \\
-\mathcal{C}_{\theta_{e}} & 0
\end{array}\right]\left[\begin{array}{l}
\boldsymbol{z} \\
h
\end{array}\right]-\left[\begin{array}{cc}
\mathcal{N}_{q} & 0 \\
0 & \mathcal{M}_{q}[\boldsymbol{z}]
\end{array}\right]\left[\begin{array}{l}
\boldsymbol{z} \\
h
\end{array}\right]+\left[\begin{array}{c}
P_{q}(m \boldsymbol{u}) \\
-\mathcal{B}_{q}(h-D v)
\end{array}\right] \text { in } \boldsymbol{W}_{\sigma}^{q}(\Omega) \\
{\left[\begin{array}{l}
\boldsymbol{z}(0) \\
h(0)
\end{array}\right]=\left[\begin{array}{l}
\boldsymbol{z}_{0} \\
h_{0}
\end{array}\right] \in \boldsymbol{W}_{\sigma}^{q}(\Omega),}
\end{array}\right.
$$

or alternatively

$$
\begin{gathered}
\frac{d}{d t}\left[\begin{array}{l}
\boldsymbol{z} \\
h
\end{array}\right]=\left[\begin{array}{cc}
\mathcal{A}_{q} & -\mathcal{C}_{\gamma} \\
-\mathcal{C}_{\theta_{e}} & -\mathcal{B}_{q}
\end{array}\right]\left[\begin{array}{l}
\boldsymbol{z} \\
h
\end{array}\right]-\left[\begin{array}{cc}
\mathcal{N}_{q} & 0 \\
0 & \mathcal{M}_{q}[\boldsymbol{z}]
\end{array}\right]\left[\begin{array}{c}
\boldsymbol{z} \\
h
\end{array}\right]+\left[\begin{array}{c}
P_{q}(m \boldsymbol{u}) \\
\mathcal{B}_{q} D v
\end{array}\right] \\
\boldsymbol{W}_{\sigma}^{q}(\Omega) \equiv \boldsymbol{L}_{\sigma}^{q}(\Omega) \times L^{q}(\Omega) .
\end{gathered}
$$

\subsection{The linearized $w$-problem of the translated $z$-model.}

Next, still for $1<q<\infty$, we introduce the linearized controlled system of the translated PDE-model (1.13), in the variable $\boldsymbol{w}=\left\{\boldsymbol{w}_{f}, w_{h}\right\} \in \boldsymbol{L}_{\sigma}^{q}(\Omega) \times L^{q}(\Omega) \equiv \boldsymbol{W}_{\sigma}^{q}(\Omega)$ :

$$
\left\{\begin{array}{rlrl}
\frac{d \boldsymbol{w}_{f}}{d t}-\nu \Delta \boldsymbol{w}_{f}+L_{e}\left(\boldsymbol{w}_{f}\right)-\gamma w_{h} \boldsymbol{e}_{d}+\nabla \chi & =m \boldsymbol{u} & & \text { in } Q \\
\frac{d w_{h}}{d t}-\kappa \Delta w_{h}+\boldsymbol{y}_{e} \cdot \nabla w_{h}+\boldsymbol{w}_{f} \cdot \nabla \theta_{e} & =0 & & \text { in } Q \\
\operatorname{div} \boldsymbol{w}_{f} & =0 & \text { in } Q \\
\boldsymbol{w}_{f} \equiv 0, w_{h} & \equiv v & & \text { on } \Sigma \\
\boldsymbol{w}_{f}(0, \cdot)=\boldsymbol{w}_{f, 0} ; \quad w_{h}(0, \cdot) & =w_{h, 0} & & \text { on } \Omega .
\end{array}\right.
$$

with I.C. $\left\{\boldsymbol{w}_{f}(0), w_{h}(0)\right\} \in \boldsymbol{W}_{\sigma}^{q}(\Omega)=\boldsymbol{L}_{\sigma}^{q}(\Omega) \times L^{q}(\Omega)$. Its corresponding abstract version is, referring also to (1.20), (1.29a-b) rewritten, for $\boldsymbol{w}=\left\{\boldsymbol{w}_{f}, w_{h}\right\}$ as

$$
\begin{gathered}
\frac{d \boldsymbol{w}}{d t}=\left[\begin{array}{c}
\frac{d \boldsymbol{w}_{f}}{d t} \\
\frac{d w_{h}}{d t}
\end{array}\right]=\left[\begin{array}{c}
\mathcal{A}_{q} \boldsymbol{w}_{f}-\mathcal{C}_{\gamma} w_{h}+P_{q}(m \boldsymbol{u}) \\
-\mathcal{B}_{q}\left(w_{h}-D v\right)-\mathcal{C}_{\theta_{e}} \boldsymbol{w}_{f}
\end{array}\right] \text { in } \boldsymbol{W}_{\sigma}^{q}(\Omega) \\
\frac{d \boldsymbol{w}}{d t}=\frac{d}{d t}\left[\begin{array}{l}
\boldsymbol{w}_{f} \\
w_{h}
\end{array}\right]=\left[\begin{array}{cc}
\mathcal{A}_{q} & -\mathcal{C}_{\gamma} \\
-\mathcal{C}_{\theta_{e}} & 0
\end{array}\right]\left[\begin{array}{l}
\boldsymbol{w}_{f} \\
w_{h}
\end{array}\right]+\left[\begin{array}{c}
P_{q}(m \boldsymbol{u}) \\
-\mathcal{B}_{q}\left(w_{h}-D v\right)
\end{array}\right] \text { in } \boldsymbol{W}_{\sigma}^{q}(\Omega)
\end{gathered}
$$

We may also write

$$
\frac{d \boldsymbol{w}}{d t}=\left[\begin{array}{c}
\frac{d \boldsymbol{w}_{f}}{d t} \\
\frac{d w_{h}}{d t}
\end{array}\right]=\left[\begin{array}{cc}
\mathcal{A}_{q} & -\mathcal{C}_{\gamma} \\
-\mathcal{C}_{\theta_{e}} & -\mathcal{B}_{q}
\end{array}\right]\left[\begin{array}{c}
\boldsymbol{w}_{f} \\
w_{h}
\end{array}\right]+\left[\begin{array}{c}
P_{q}(m \boldsymbol{u}) \\
\mathcal{B}_{q} D v
\end{array}\right] \text { in } \boldsymbol{L}_{\sigma}^{q}(\Omega) \times\left[\mathcal{D}\left(B_{q}^{*}\right)\right]^{\prime}
$$


The corresponding linearized uncontrolled problem $\{v \equiv 0, \boldsymbol{u} \equiv 0\}$ is

$$
\begin{gathered}
\frac{d \boldsymbol{w}}{d t}=\frac{d}{d t}\left[\begin{array}{l}
\boldsymbol{w}_{f} \\
w_{h}
\end{array}\right]=\mathbb{A}_{q}\left[\begin{array}{l}
\boldsymbol{w}_{f} \\
w_{h}
\end{array}\right]=\left[\begin{array}{cc}
\mathcal{A}_{q} & -\mathcal{C}_{\gamma} \\
-\mathcal{C}_{\theta_{e}} & -\mathcal{B}_{q}
\end{array}\right]\left[\begin{array}{l}
\boldsymbol{w}_{f} \\
w_{h}
\end{array}\right] \\
\mathbb{A}_{q}=\left[\begin{array}{cc}
\mathcal{A}_{q} & -\mathcal{C}_{\gamma} \\
-\mathcal{C}_{\theta_{e}} & -\mathcal{B}_{q}
\end{array}\right]: \boldsymbol{W}_{\sigma}^{q}(\Omega) \equiv \boldsymbol{L}_{\sigma}^{q}(\Omega) \times L^{q}(\Omega) \supset \mathcal{D}\left(\mathbb{A}_{q}\right)=\mathcal{D}\left(\mathcal{A}_{q}\right) \times \mathcal{D}\left(\mathcal{B}_{q}\right) \\
=\left(\boldsymbol{W}^{2, q}(\Omega) \cap \boldsymbol{W}_{0}^{1, q}(\Omega) \cap \boldsymbol{L}_{\sigma}^{q}(\Omega)\right) \times\left(W^{2, q}(\Omega) \cap W_{0}^{1, q}(\Omega)\right) \longrightarrow \boldsymbol{W}_{\sigma}^{q}(\Omega) .
\end{gathered}
$$

Properties of the operator $\mathbb{A}_{q}$ in (1.34)-critical for the proper setting of the present stabilization analysis will be given in the next Section 1.7.

\subsection{Properties of the operator $\mathbb{A}_{q}$ in (1.34).}

We shall use throughout the following notation (recall (1.3), (1.5), (1.32) and (1.11)

$$
\boldsymbol{W}_{\sigma}^{q}(\Omega) \equiv \boldsymbol{L}_{\sigma}^{q}(\Omega) \times L^{q}(\Omega) ; \quad \boldsymbol{V}^{q, p}(\Omega) \equiv \widetilde{\boldsymbol{B}}_{q, p}^{2-^{2 / p}}(\Omega) \times L^{q}(\Omega) .
$$

Remark 1.3. By using the maximal regularity of the heat equation, instead of the state space $\boldsymbol{V}^{q, p}(\Omega)$ in (1.3), (1.35) we could take the state space $\boldsymbol{V}_{b}^{q, p}(\Omega)$ to be the product of two Besov spaces, i.e. $\boldsymbol{V}_{b}^{q, p}(\Omega) \equiv$ $\widetilde{\boldsymbol{B}}_{q, p}^{2-{ }^{2 / p}}(\Omega) \times B_{q, p}^{2-2 / p}(\Omega)$ as in $(1.3 \mathrm{~b})$. Here, the second Besov component is the real interpolation between $L^{q}(\Omega)$ and $\mathcal{D}\left(\mathcal{B}_{q}\right)$, see $\left[70\right.$. This remark applies to all results involving $\boldsymbol{V}^{q, p}(\Omega)$, but it will not necessarily be noted explicitly case by case, in order not to overload the notation.

Accordingly, we shall look at the operator $\mathbb{A}_{q}$ in (1.34) as defined on either space

$$
\mathbb{A}_{q}: \boldsymbol{W}_{\sigma}^{q}(\Omega) \supset \mathcal{D}\left(\mathbb{A}_{q}\right) \rightarrow \boldsymbol{W}_{\sigma}^{q}(\Omega) \text { or } \mathbb{A}_{q}: \boldsymbol{V}^{q, p}(\Omega) \supset \mathcal{D}\left(\mathbb{A}_{q}\right) \rightarrow \boldsymbol{V}^{q, p}(\Omega) .
$$

The following result collects basic properties of the operator $\mathbb{A}_{q}$. It is essentially a corollary of Theorems A.3 and A.4 in Appendix $\mathrm{A}$ for the Oseen operator $\mathcal{A}_{q}$, as similar results hold for the operator $\mathcal{B}_{q}$, while the operator $\mathcal{C}_{\gamma}$ and $\mathcal{C}_{\theta_{e}}$ in the definition (1.32) of $\mathbb{A}_{q}$ are bounded operators, see (1.21), (1.22).

Theorem 1.2. With reference to the Operator $\mathbb{A}_{q}$ in (1.34), (1.36), the following properties hold true:

(i) $\mathbb{A}_{q}$ is the generator of strongly continuous analytic semigroup on either $\boldsymbol{W}_{\sigma}^{q}(\Omega)$ or $\boldsymbol{V}^{q, p}(\Omega)$ or $\boldsymbol{V}_{b}^{q, p}(\Omega)$ for $t>0$

(ii) $\mathbb{A}_{q}$ possesses the $L^{p}$-maximal regularity property on either $\boldsymbol{W}_{\sigma}^{q}(\Omega)$ or $\boldsymbol{V}^{q, p}(\Omega)$ or $\boldsymbol{V}_{b}^{q, p}(\Omega)$ over a finite interval:

$$
\mathbb{A}_{q} \in M \operatorname{Reg}\left(L^{p}(0, T ; *)\right), 0<T<\infty, \quad(*)=\boldsymbol{W}_{\sigma}^{q}(\Omega) \text { or } \boldsymbol{V}^{q, p}(\Omega) .
$$

(iii) $\mathbb{A}_{q}$ has compact resolvent on either $\boldsymbol{W}_{\sigma}^{q}(\Omega)$ or $\boldsymbol{V}^{q, p}(\Omega)$.

Analyticity of $e^{\mathcal{A}_{q} t}\left(\right.$ resp. $\left.e^{\mathcal{B}_{q} t}\right)$ in $\boldsymbol{L}_{\sigma}^{q}(\Omega)$ (resp. $L^{q}(\Omega)$ ) implies analyticity of $e^{\mathcal{A}_{q} t}$ (resp. $\left.e^{\mathcal{B}_{q} t}\right)$ on $\mathcal{D}\left(\mathcal{A}_{q}\right)=\mathcal{D}\left(A_{q}\right)$ (resp. $\mathcal{D}\left(\mathcal{B}_{q}\right)=\mathcal{D}\left(B_{q}\right)$ ), hence analyticity of $e^{\mathcal{A}_{q} t}$ (resp. $e^{\mathcal{B}_{q} t}$ ) on the interpolation space $\widetilde{\boldsymbol{B}}_{q, p}^{22^{2} / p}(\Omega)$ in (1.11). (or in $\boldsymbol{B}_{q, p}^{2-^{2} / p}(\Omega)$ ) in (1.9) (resp. (1.4) in the scalar case).

For the notation of, and the results on, maximal regularity, see [1, [23, [32, 37], [53, [54, 66], [93], 91, etc. In particular, we recall that on a Banach space, maximal regularity implies analyticity of the semigroup, 22] but not conversely [23, [54. We refer to Appendix A. 
Basic assumption: By Theorem 1.2, the operator $\mathbb{A}_{q}$ in (1.34) has the eigenvalues (spectrum) located in a triangular sector of well-known type. Then our basic assumption - which justifies the present paper - is that such operator $\mathbb{A}_{q}$ is unstable: that is, $\mathbb{A}_{q}$ has a finite number, say $N$, of eigenvalues $\lambda_{1}, \lambda_{2}, \lambda_{3}, \ldots, \lambda_{N}$ on the complex half plane $\{\lambda \in \mathbb{C}: \operatorname{Re} \lambda \geq 0\}$ which we then order according to their real parts, so that

$$
\cdots \leq \operatorname{Re} \lambda_{N+1}<0 \leq \operatorname{Re} \lambda_{N} \leq \ldots \leq \operatorname{Re} \lambda_{1},
$$

each $\lambda_{i}, i=1, \ldots, N$, being an unstable eigenvalue repeated according to its geometric multiplicity $\ell_{i}$. Let $M$ denote the number of distinct unstable eigenvalues $\lambda_{i}$ of $\mathbb{A}_{q}$, so that $\ell_{i}$ is equal to the dimension of the eigenspace corresponding to $\lambda_{i}$. Instead, $N=\sum_{i=1}^{M} N_{i}$ is the sum of the corresponding algebraic multiplicity $N_{i}$ of $\lambda_{i}$, where $N_{i}$ is the dimension of the corresponding generalized eigenspace.

Remark 1.4. Condition (1.38) is intrinsic to the notion of 'stabilization', whereby then one seeks to construct a feedback control that transforms an original unstable problem (with no control) into a stable one. However, as is well-known [60], the same entire procedure can be employed to enhance at will the stability of an originally stable system $\left(\operatorname{Re} \lambda_{1}<0\right)$ by feedback control. This is the case of Remark 1.1(i).

\section{Main results.}

As in our past work [9], [10], [11], [12, [57], [58], 60], 61], we shall henceforth let $\boldsymbol{L}_{\sigma}^{q}(\Omega)$ denote the complexified space $\boldsymbol{L}_{\sigma}^{q}(\Omega)+i \boldsymbol{L}_{\sigma}^{q}(\Omega)$, and similarly for $L^{q}(\Omega)$, whereby then we consider the extension of the linearized problem (1.32) to such complexified space $\boldsymbol{L}_{\sigma}^{q}(\Omega) \times L^{q}(\Omega)$. Thus, henceforth, $\boldsymbol{w}$ will mean $\boldsymbol{w}+i \widetilde{\boldsymbol{w}}, \boldsymbol{u}$ will mean $\boldsymbol{u}+i \widetilde{\boldsymbol{u}}, \boldsymbol{w}_{0}$ will mean $\boldsymbol{w}_{0}+i \widetilde{\boldsymbol{w}}_{0}$. Our results would be given in this complexified setting. How to return to the real-valued formulation of the results was done in these past reference (see e.g. [9], 10], [57, [60, Section 2.7]). Because of space constraints, such real-valued statements will not be explicitly listed on the present paper. We refer to the above references.

\subsection{Orientation.}

A main additional feature of the results below is that the feedback control $\boldsymbol{u}_{k}$ corresponding to the fluid equation is of reduced dimension: that is, of dimension $(d-1)$ rather than of dimension $d$. More precisely, setting $\left.\boldsymbol{u} \overline{=\left\{u^{(1)}\right.}, u^{(2)}, \ldots, u^{(d)}\right\}$ to express the vector control $\boldsymbol{u}$ acting on $\omega$ in terms of its $d$ coordinates, the only constraint is that the last component $u^{(d)}$ is always needed, along with additional $(d-2)$ components with no preference. Thus, for $d=2$, a feedback control $\boldsymbol{u}$ may be used invoking only the component $u^{(2)}$, with no need of component $u^{(1)}$. For $d=3$, a feedback control $\boldsymbol{u}$ may be used involving either the components $\left\{u^{(1)}, u^{(3)}\right\}$ or else components $\left\{u^{(2)}, u^{(3)}\right\}$. This is due to the UCP of Theorem B.1 reported in Appendix B To express above facts, we introduce appropriate notation. Recall that the vector $\{\boldsymbol{\varphi}, \psi\}=\left\{\varphi^{(1)}, \varphi^{(2)}, \ldots, \varphi^{(d)}, \psi\right\}$ in $\boldsymbol{W}_{\sigma}^{q}(\Omega) \equiv \boldsymbol{L}_{\sigma}^{q}(\Omega) \times L^{q}(\Omega)$ has $(d+1)$ coordinates, the first $d$ coordinates corresponds to the fluid space, while the last coordinate corresponds to heat space. Motivated by the above considerations, ultimately by the UCP of Theorem B.1 of Appendix B, we shall introduce the notation $\widehat{\boldsymbol{L}}_{\sigma}^{q}(\Omega)$ to denote

$$
\begin{aligned}
& \widehat{\boldsymbol{L}}_{\sigma}^{q}(\Omega) \equiv \text { any }(d-1) \text {-dimensional sub-space obtained from } \boldsymbol{L}_{\sigma}^{q}(\Omega) \\
& \text { after omitting one specific coordinate, except the } d^{\text {th }} \text { coordinate, }
\end{aligned}
$$

from the vectors of $\boldsymbol{L}_{\sigma}^{q}(\Omega)$. 
2.2 Global well-posedness and uniform exponential stabilization of the linearized $\boldsymbol{w}$-problem (1.32) on either the space $\boldsymbol{W}_{\sigma}^{q}(\Omega) \equiv \boldsymbol{L}_{\sigma}^{q}(\Omega) \times L^{q}(\Omega)$ or the space $\boldsymbol{V}^{q, p}(\Omega) \equiv \widetilde{\boldsymbol{B}}_{q, p}^{2-2 / p}(\Omega) \times L^{q}(\Omega), 1<q<\infty, 1<p<{ }^{2 q} / 2 q-1$.

Theorem 2.1. Let the operator $\mathbb{A}_{q}$ in (1.34) have $N$ possibly repeated unstable eigenvalues $\left\{\lambda_{j}\right\}_{j=1}^{N}$ as in (1.38), of which $M$ are distinct. Let $\ell_{i}$ denote the geometric multiplicity of $\lambda_{i}$. Set $K=\sup \left\{\ell_{i} ; i=\right.$ $1, \ldots, M\}$. Let $\left(\boldsymbol{W}_{\sigma}^{q}\right)_{N}^{u}$ be the $N$-dimensional subspace of $\boldsymbol{W}_{\sigma}^{q}(\Omega)$ defined in (3.2) below, which is the generalized eigenspace of $\mathbb{A}_{q}$ corresponding to the unstable eigenvalues $\left\{\lambda_{j}\right\}_{j=1}^{N}$. Recall the space $\widehat{\boldsymbol{L}}_{\sigma}^{q}(\Omega)$ from (2.1) and let likewise $\left(\widehat{\boldsymbol{W}}_{\sigma}^{q}\right)_{N}^{u}$ be any space obtained from $\left(\boldsymbol{W}_{\sigma}^{q}\right)_{N}^{u}$ by omitting one specific coordinate from the vectors of $\left(\boldsymbol{W}_{\sigma}^{q}\right)_{N}^{u}$ except the $d^{\text {th }}$ coordinate. Then, one may construct finite dimensional feedback operators $F$ and $J$, as desired

$$
\begin{aligned}
v & =F \boldsymbol{w}=\sum_{k=1}^{K}\left\langle P_{N} \boldsymbol{w}, \boldsymbol{p}_{k}\right\rangle f_{k}, f_{k} \in \mathcal{F} \subset W^{2-1 / q}, q(\Omega), \\
\boldsymbol{p}_{k} & \in\left(\boldsymbol{W}_{N}^{u}\right)^{*} \subset \boldsymbol{L}_{\sigma}^{q^{\prime}}(\Omega) \times L^{q}(\Omega), q \geq 2, f_{k} \text { supported on } \widetilde{\Gamma} . \\
D v=D F \boldsymbol{w} & \in \boldsymbol{W}^{2, q}(\Omega)
\end{aligned}
$$

$$
\begin{aligned}
J \boldsymbol{w}=P_{q} m(\boldsymbol{u})=P_{q} m\left(\sum_{k=1}^{K}\left\langle P_{N} \boldsymbol{w}, \boldsymbol{q}_{k}\right\rangle \boldsymbol{u}_{k}\right), \boldsymbol{u}_{k} \in \widehat{\boldsymbol{L}}_{\sigma}^{q}(\Omega), \\
\qquad \boldsymbol{q}_{k} \in\left(\boldsymbol{W}_{N}^{u}\right)^{*} \subset \boldsymbol{L}_{\sigma}^{q^{\prime}}(\Omega) \times L^{q}(\Omega), \boldsymbol{u}_{k} \text { supported on } \omega .
\end{aligned}
$$

such that, with $\boldsymbol{w}=\left\{\boldsymbol{w}_{f}, w_{h}\right\}, \boldsymbol{w}_{N}=P_{N} \boldsymbol{w}, P_{N}$ the projector in (3.1a), once inserted in the $\boldsymbol{w}$-problem (1.32), yield a resulting closed-loop linearized $\boldsymbol{w}$-problem in feedback form

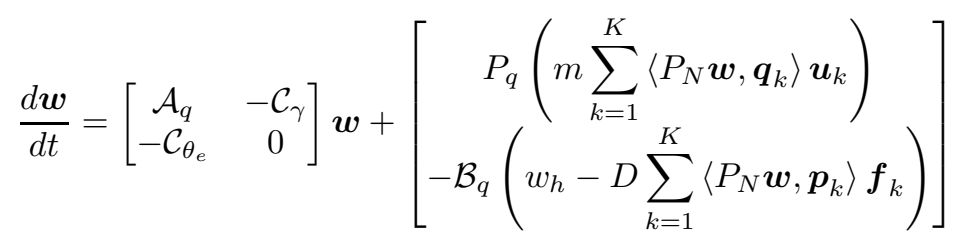

or by (2.2), (2.3)

$$
\frac{d \boldsymbol{w}}{d t}=\left[\begin{array}{cc}
\mathcal{A}_{q} & -\mathcal{C}_{\gamma} \\
-\mathcal{C}_{\theta_{e}} & 0
\end{array}\right] \boldsymbol{w}+\left[\begin{array}{c}
J \boldsymbol{w} \\
-\mathcal{B}_{q}\left(w_{h}-D F \boldsymbol{w}\right)
\end{array}\right] \equiv \mathbb{A}_{F, q} \boldsymbol{w}
$$

This way, the feedback operator $\mathbb{A}_{F, q}$ is defined for the linearized $\boldsymbol{w}$-problem in feedback form, as

$$
\mathbb{A}_{F, q}=\hat{\mathbb{A}}_{F, q}+\Pi
$$

so that (2.4b) is rewritten as

$$
\begin{gathered}
\frac{d \boldsymbol{w}}{d t}=\mathbb{A}_{F, q} \boldsymbol{w}=\hat{\mathbb{A}}_{F, q} \boldsymbol{w}+\Pi \boldsymbol{w} \\
\hat{\mathbb{A}}_{F, q} \boldsymbol{w}=\left[\begin{array}{c}
-A_{q} \boldsymbol{w}_{1} \\
-\mathcal{B}_{q}\left(w_{2}-D F \boldsymbol{w}\right)
\end{array}\right], \quad \Pi \boldsymbol{w}=\left[\begin{array}{cc}
A_{o, q} & -\mathcal{C}_{\gamma} \\
-\mathcal{C}_{\theta_{e}} & 0
\end{array}\right] \boldsymbol{w}+\left[\begin{array}{c}
J \boldsymbol{w} \\
0
\end{array}\right] \\
\mathcal{D}\left(\mathbb{A}_{F, q}\right)=\mathcal{D}\left(\hat{\mathbb{A}}_{F, q}\right)=\left\{\boldsymbol{w}=\left[\begin{array}{l}
\boldsymbol{w}_{1} \\
w_{2}
\end{array}\right] \in \boldsymbol{W}_{\sigma}^{q}(\Omega)=\boldsymbol{L}_{\sigma}^{q}(\Omega) \times L^{q}(\Omega):\right. \\
\left.\boldsymbol{w}_{1} \in \mathcal{D}\left(A_{q}\right),\left(w_{2}-D F \boldsymbol{w}\right) \in \mathcal{D}\left(\mathcal{B}_{q}\right)=\mathcal{D}\left(B_{q}\right)\right\}
\end{gathered}
$$




$$
\mathcal{D}\left(\mathbb{A}_{F}\right) \subset \mathcal{D}\left(A_{q}\right) \times \boldsymbol{W}^{2, q}(\Omega), \mathcal{D}(\Pi)=\mathcal{D}\left(A_{o, q}\right) \times L^{q}(\Omega)
$$

recalling (1.15), (1.17), (1.18), (1.23c), (2.2b). Moreover;

(i) The operator $\mathbb{A}_{F, q}$ in (2.4), (2.6) generates a s.c. analytic semigroup $e^{\mathbb{A}_{F, q} t}$ on $\boldsymbol{W}_{\sigma}^{q}(\Omega) \equiv \boldsymbol{L}_{\sigma}^{q}(\Omega) \times$ $L^{q}(\Omega)$ as well as in the space $\boldsymbol{V}^{q, p}(\Omega) \equiv \widetilde{B}_{q, p}^{2-2 / p} \times L^{q}(\Omega)$, or $\boldsymbol{V}_{b}^{q, p}(\Omega)$ see also Remark 1.3 .

(ii) Such semigroup $e^{\mathbb{A}_{F, q} t}$ is uniformly (exponentially) stable in either of these spaces

$$
\left\|e^{\mathbb{A}_{F, q} t} \boldsymbol{w}_{0}\right\|_{(\cdot)} \leq C_{\gamma_{0}} e^{-\gamma_{0} t}\left\|\boldsymbol{w}_{0}\right\|_{(\cdot)}, t \geq 0
$$

where $(\cdot)$ denotes either $\boldsymbol{L}_{\sigma}^{q}(\Omega) \times L^{q}(\Omega) \equiv \boldsymbol{W}_{\sigma}^{q}(\Omega)$ or else $\widetilde{\boldsymbol{B}}_{q, p}^{2-2 / p}(\Omega) \times L^{q}(\Omega) \equiv \boldsymbol{V}^{q, p}(\Omega)$, or $\boldsymbol{V}_{b}^{q, p}(\Omega)$. In (2.7), $\gamma_{0}$ is any positive number such that Re $\lambda_{N+1}<-\gamma_{0}<0$.

(iii) Finally, $\mathbb{A}_{F, q}$ has maximal $L^{p}$-regularity up to $T=\infty$ on either of these spaces:

$$
\left\{\begin{array}{c}
\mathbb{A}_{F, q} \in M \operatorname{Reg}\left(L^{p}(0, \infty ; \cdot)\right) \text {, where }(\cdot) \text { denotes } \\
\text { either } \boldsymbol{L}_{\sigma}^{q}(\Omega) \times L^{q}(\Omega) \equiv \boldsymbol{W}_{\sigma}^{q}(\Omega) \\
\text { or else } \widetilde{\boldsymbol{B}}_{q, p}^{2-2 / p}(\Omega) \times L^{q}(\Omega) \equiv \boldsymbol{V}^{q, p}(\Omega) \text {, or } \boldsymbol{V}_{b}^{q, p}(\Omega) .
\end{array}\right.
$$

The proof of Theorem 2.1 begins in Section 3 and proceeds through Section [7. Such proof gives the feedback vectors $\boldsymbol{u}_{k}$ in (2.3) as being in $\boldsymbol{L}_{\sigma}^{q}(\Omega)$. The refinement of asserting that such vectors $\boldsymbol{u}_{k}$ can be taken in $\widehat{\boldsymbol{L}}_{\sigma}^{q}(\Omega)$ defined in (2.1) is given in Appendix C. More precisely, analyticity in (i) is proved in Theorem 6.1, uniform decay in (ii) is proved in Theorem 6.2, while maximal $L^{p}$-regularity in (iii) is established in Theorem 7.1 .

\subsection{Local well-posedness and uniform (exponential) null stabilization of the} translated nonlinear $\{\boldsymbol{z}, h\}$-problem (1.29) or (1.13) by means of a finite dimensional explicit, spectral based feedback control pair $\{v, u\}$ localized on $\{\widetilde{\Gamma}, \omega\}$.

Starting with the present subsection, the nonlinearity of problem (1.1) will impose for $d=3$ the requirement $q>3$, see (8.24) below. As our deliberate goal is to obtain the stabilization result for the fluid component $\boldsymbol{y}$ in the space $\widetilde{\boldsymbol{B}}_{q, p}^{2-2 / p}(\Omega)$ which does not recognize boundary conditions, Remark [1.2] then the limitation $p<{ }^{2 q} / 2 q-1$ of this space applies, see (1.11). In conclusion, our well-posedness and stabilization results will hold under the restriction $q>3,1<p<6 / 5$ for $d=3$, and $q>2,1<p<4 / 3$ for $d=2$.

Theorem 2.2. Let $d=2,3, q>d, 1<p<\frac{2 q}{2 q-1}$. Consider the nonlinear open-loop $\{\boldsymbol{z}, h\}$-problem (1.29a)

$$
\left\{\begin{array}{l}
\frac{d}{d t}\left[\begin{array}{l}
\boldsymbol{z} \\
h
\end{array}\right]=\left[\begin{array}{cc}
\mathcal{A}_{q} & -\mathcal{C}_{\gamma} \\
-\mathcal{C}_{\theta_{e}} & 0
\end{array}\right]\left[\begin{array}{c}
\boldsymbol{z} \\
h
\end{array}\right]-\left[\begin{array}{cc}
\mathcal{N}_{q} & 0 \\
0 & \mathcal{M}_{q}[\boldsymbol{z}]
\end{array}\right]\left[\begin{array}{c}
\boldsymbol{z} \\
h
\end{array}\right]+\left[\begin{array}{c}
P_{q}(m \boldsymbol{u}) \\
-\mathcal{B}_{q}(h-D v)
\end{array}\right] \text { in } \boldsymbol{W}_{\sigma}^{q}(\Omega) \\
{\left[\begin{array}{c}
\boldsymbol{z}(0) \\
h(0)
\end{array}\right]=\left[\begin{array}{c}
\boldsymbol{z}_{0} \\
h_{0}
\end{array}\right] \in \boldsymbol{W}_{\sigma}^{q}(\Omega)}
\end{array}\right.
$$

which, upon application of feedback control pair $\left\{v, P_{q} m(\boldsymbol{u})\right\}=\left\{F\left[\begin{array}{l}\boldsymbol{z} \\ h\end{array}\right], J\left[\begin{array}{l}\boldsymbol{z} \\ h\end{array}\right]\right\}$ of the same structure 
(2.2a), (2.3) as for the linearized feedback system (1.32b)

$$
\left[\begin{array}{c}
P_{q} m(\boldsymbol{u}) \\
v
\end{array}\right]=\left[\begin{array}{c}
J\left[\begin{array}{l}
\boldsymbol{z} \\
h
\end{array}\right] \\
F\left[\begin{array}{l}
\boldsymbol{z} \\
h
\end{array}\right]
\end{array}\right]=\left[\begin{array}{c}
P_{q}\left(m\left(\sum_{k=1}^{K}\left\langle P_{N}\left[\begin{array}{l}
\boldsymbol{z} \\
h
\end{array}\right], \boldsymbol{q}_{k}\right\rangle \boldsymbol{u}_{k}\right)\right) \\
\sum_{k=1}^{K}\left\langle P_{N}\left[\begin{array}{l}
\boldsymbol{z} \\
h
\end{array}\right], \boldsymbol{p}_{k}\right\rangle f_{k}
\end{array}\right]
$$

$P_{N}$ defined in (3.1a) is rewritten in a closed loop feedback form as

$$
\left\{\begin{aligned}
\frac{d \boldsymbol{z}}{d t}-\mathcal{A}_{q} \boldsymbol{z}+\mathcal{C}_{\gamma} h+\mathcal{N}_{q} \boldsymbol{z} & =P_{q}\left(m\left(\sum_{k=1}^{K}\left\langle P_{N}\left[\begin{array}{c}
\boldsymbol{z} \\
h
\end{array}\right], \boldsymbol{q}_{k}\right\rangle \boldsymbol{u}_{k}\right)\right) \\
\frac{d h}{d t}+\mathcal{C}_{\theta_{e}} \boldsymbol{z}+\mathcal{M}_{q}[\boldsymbol{z}] h & =-\mathcal{B}_{q}\left(h-D \sum_{k=1}^{K}\left\langle P_{N}\left[\begin{array}{c}
\boldsymbol{z} \\
h
\end{array}\right], \boldsymbol{p}_{k}\right\rangle f_{k}\right) .
\end{aligned}\right.
$$

or in matrix form, recalling (2.4b), 2.6b)

$$
\begin{aligned}
\frac{d}{d t}\left[\begin{array}{l}
\boldsymbol{z} \\
h
\end{array}\right]=\mathbb{A}_{F, q}\left[\begin{array}{l}
\boldsymbol{z} \\
h
\end{array}\right]-\left[\begin{array}{cc}
\mathcal{N}_{q} & 0 \\
0 & \mathcal{M}_{q}[\boldsymbol{z}]
\end{array}\right]\left[\begin{array}{l}
\boldsymbol{z} \\
h
\end{array}\right] ; \mathbb{A}_{F, q}\left[\begin{array}{l}
\boldsymbol{z} \\
h
\end{array}\right]=\left[\begin{array}{cc}
\mathcal{A}_{q} & -\mathcal{C}_{\gamma} \\
-\mathcal{C}_{\theta_{e}} & 0
\end{array}\right]\left[\begin{array}{l}
\boldsymbol{z} \\
h
\end{array}\right] \\
+\left[\begin{array}{c}
J\left[\begin{array}{l}
\boldsymbol{z} \\
h
\end{array}\right] \\
-\mathcal{B}_{q}\left(h-D F\left[\begin{array}{l}
\boldsymbol{z} \\
h
\end{array}\right]\right)
\end{array}\right] .
\end{aligned}
$$

Let $d=2,3, q>d, 1<p<\frac{2 q}{2 q-1}$. There exists a positive constant $r_{1}>0$ (identified in the proof below in (8.31) such that if

$$
\left\|\left\{\boldsymbol{z}_{0}, h_{0}\right\}\right\|_{\widetilde{\boldsymbol{B}}_{q, p}^{2-2 / p}(\Omega) \times B_{q, p}^{2-2 / p}(\Omega)}<r_{1},
$$

then $E q$ (2.11), or (2.12) has a unique fixed point non-linear semigroup solution,

$$
\begin{gathered}
{\left[\begin{array}{l}
\boldsymbol{z} \\
h
\end{array}\right](t)=e^{\mathbb{A}_{F, q} t}\left[\begin{array}{c}
\boldsymbol{z}_{0} \\
h_{0}
\end{array}\right]-\int_{0}^{t} e^{\mathbb{A}_{F, q}(t-\tau)}\left[\begin{array}{c}
\mathcal{N}_{q} \boldsymbol{z}(\tau) \\
\mathcal{M}_{q}[\boldsymbol{z}] h(\tau)
\end{array}\right] d \tau, \text { in the space } \boldsymbol{X}_{p, q, \sigma}^{\infty} \times X_{p, q}^{\infty}} \\
\{\boldsymbol{z}, h\} \in \boldsymbol{X}_{p, q, \sigma}^{\infty} \times X_{p, q}^{\infty} \equiv L^{p}\left(0, \infty ; \mathcal{D}\left(\mathbb{A}_{F, q}\right)\right) \cap W^{1, p}\left(0, \infty ; \boldsymbol{W}_{\sigma}^{q}(\Omega)\right),
\end{gathered}
$$

Moreover, (referred to as trace theorem)

$$
\begin{gathered}
\boldsymbol{X}_{p, q, \sigma}^{\infty} \hookrightarrow C\left([0, \infty] ; \boldsymbol{B}_{q, p}^{2-2 / p}(\Omega)\right) \\
X_{p, q}^{\infty} \hookrightarrow C\left([0, \infty] ; B_{q, p}^{2-2 / p}(\Omega)\right)
\end{gathered}
$$

so that

$$
\boldsymbol{X}_{p, q, \sigma}^{\infty} \times X_{p, q}^{\infty} \hookrightarrow C\left([0, \infty] ; \boldsymbol{V}_{b}^{q, p}(\Omega)\right)
$$

The space $\boldsymbol{X}_{p, q, \sigma}^{\infty} \times X_{p, q}^{\infty}$ defined above is the space of $L^{p}$-maximal regularity for the generator $\mathbb{A}_{F, q}$.

Theorem 2.3. Assume the setting of Theorem [2.2, in particular $d=2,3, q>d, 1<p<\frac{2 q}{2 q-1}$ and the smallness condition (2.13) for the I.C. Then, the solution guaranteed by Theorem 2.2 is uniformly stable in the space $\boldsymbol{V}_{b}^{q, p}(\Omega) \equiv \widetilde{\boldsymbol{B}}_{q, p}^{2-2 / p}(\Omega) \times B_{q, p}^{2-2^{2 / p}}(\Omega)$, see also Remark 1.3; there exists $\widetilde{\gamma}>0, M_{\widetilde{\gamma}}>0$ such that said solution satisfies

$$
\left\|\left[\begin{array}{c}
\boldsymbol{z} \\
h
\end{array}\right](t)\right\|_{\boldsymbol{V}_{b}^{q, p}(\Omega)} \leq M_{\widetilde{\gamma}} e^{-\widetilde{\gamma} t}\left\|\left[\begin{array}{c}
\boldsymbol{z}_{0} \\
h_{0}
\end{array}\right]\right\|_{\boldsymbol{V}_{b}^{q, p}(\Omega)}, \quad t \geq 0 .
$$


See Remark 9.1 for the relationship between $\gamma_{0}$ in (2.7) and $\widetilde{\gamma}$ in (2.19).

2.4 Local well-posedness and uniform (exponential) stabilization near an unstable equilibrium solution $\left\{\boldsymbol{y}_{e}, \theta_{e}\right\}$ of the original Boussinesq system (1.1) by means of a finite dimensional, explicit, spectral based feedback control pair $\{v, \boldsymbol{u}\}$ localized on $\{\widetilde{\Gamma}, \omega\}$.

The results of this subsection - the main ones of the present paper - are an immediate corollary of Theorem 2.2 and 2.3 of Section 2.3 via the change of variable (1.12).

Theorem 2.4. Let $d=2,3, q>d, 1<p<\frac{2 q}{2 q-1}$. Consider the original Boussinesq problem (1.1). Let $\left\{\boldsymbol{y}_{e}, \theta_{e}\right\}$ be a given unstable equilibrium solution as guaranteed by Theorem 1.1 for a steady state problem (1.2): i.e. assume (1.38). For a constant $\rho>0$, let the initial condition $\left\{\boldsymbol{y}_{0}, \theta_{0}\right\}$ in (1.1e) be in $\widetilde{\boldsymbol{B}}_{q, p}^{2-{ }^{2} / p}(\Omega) \times B_{q, p}^{2-2 / p}(\Omega)$ and satisfy

$$
\begin{aligned}
\mathcal{V}_{\rho}=\left\{\left\{\boldsymbol{y}_{0}, \theta_{0}\right\} \in \widetilde{\boldsymbol{B}}_{q, p}^{2-^{2 / p}}(\Omega) \times B_{q, p}^{2-2 / p}(\Omega):\right. & \\
& \left.\left\|\left\{\boldsymbol{y}_{0}, \theta_{0}\right\}-\left\{\boldsymbol{y}_{e}, \theta_{e}\right\}\right\|_{\widetilde{\boldsymbol{B}}_{q, p}^{2-2 / p}(\Omega) \times B_{q, p}^{2-2 / p}(\Omega)} \leq \rho\right\}, \rho>0 .
\end{aligned}
$$

If $\rho>0$ is sufficiently small, then: for each $\left\{\boldsymbol{y}_{0}, \theta_{0}\right\} \in \mathcal{V}_{\rho}$, let

$$
\begin{gathered}
v=F\left[\begin{array}{l}
\boldsymbol{y}-\boldsymbol{y}_{e} \\
\theta-\theta_{e}
\end{array}\right]=\sum_{k=1}^{K}\left\langle P_{N}\left[\begin{array}{l}
\boldsymbol{y}-\boldsymbol{y}_{e} \\
\theta-\theta_{e}
\end{array}\right], \boldsymbol{p}_{k}\right\rangle \boldsymbol{f}_{k} \\
\boldsymbol{u}=\widetilde{J}\left[\begin{array}{l}
\boldsymbol{y}-\boldsymbol{y}_{e} \\
\theta-\theta_{e}
\end{array}\right]=\sum_{k=1}^{K}\left\langle P_{N}\left[\begin{array}{l}
\boldsymbol{y}-\boldsymbol{y}_{e} \\
\theta-\theta_{e}
\end{array}\right], \boldsymbol{q}_{k}\right\rangle \boldsymbol{u}_{k} \\
J\left[\begin{array}{c}
\boldsymbol{y}-\boldsymbol{y}_{e} \\
\theta-\theta_{e}
\end{array}\right]=P_{q}\left(m\left(\widetilde{J}\left[\begin{array}{l}
\boldsymbol{y}-\boldsymbol{y}_{e} \\
\theta-\theta_{e}
\end{array}\right]\right)\right)
\end{gathered}
$$

that is, the finite dimensional operators $F \in \mathcal{L}\left(\boldsymbol{W}_{\sigma}^{q}(\Omega), L^{q}(\Gamma)\right)$ and $J \in \mathcal{L}\left(\boldsymbol{W}_{\sigma}^{q}(\Omega), \widehat{\boldsymbol{L}}_{\sigma}^{q}(\Omega)\right)$ be the same as in (2.2a), (2.3) for the linearized $\boldsymbol{w}$-problem (2.4); or as in (2.10) for the translated $\{\boldsymbol{z}, h\}$-problem (2.11), having the same boundary vectors $\boldsymbol{f}_{k}$ and interior vectors $\boldsymbol{p}_{k}, \boldsymbol{q}_{k} \in\left(\left(\boldsymbol{W}_{\sigma}^{q}\right)_{N}^{u}\right)^{*}$ and $\left.\boldsymbol{u}_{k} \in \widehat{\boldsymbol{L}}_{\sigma}^{q}(\Omega)\right)$ as in Theorem [2.1. Substitute $v$ in (2.21) and $\boldsymbol{u}$ in (2.22a) in (1.1d) and (1.1a) respectively, to obtain the original problem (1.11) in feedback form:

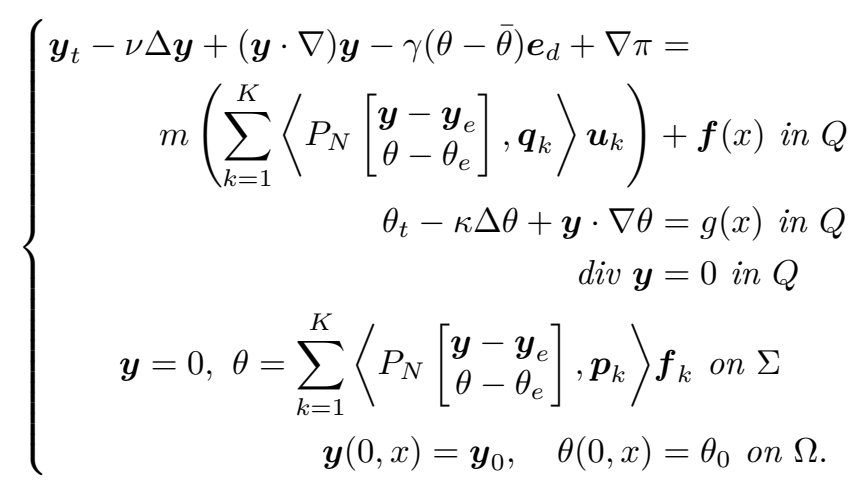


re-written abstractly after application of the Helmholtz projection $P_{q}$ on the $\boldsymbol{y}$-equation, as

$$
\left\{\begin{array}{l}
\frac{d \boldsymbol{y}}{d t}-\mathcal{A}_{q} \boldsymbol{y}+\mathcal{C}_{\gamma} \theta+\mathcal{N}_{q} \boldsymbol{y}=P_{q}\left(m\left(\sum_{k=1}^{K}\left\langle P_{N}\left[\begin{array}{c}
\boldsymbol{y}-\boldsymbol{y}_{e} \\
\theta-\theta_{e}
\end{array}\right], \boldsymbol{q}_{k}\right\rangle \boldsymbol{u}_{k}\right)\right) \\
\frac{d \theta}{d t}+\mathcal{C}_{\theta_{e}} \boldsymbol{y}+\mathcal{M}_{q}[\boldsymbol{y}] \theta=-\mathcal{B}_{q}\left(\theta-D \sum_{k=1}^{K}\left\langle P_{N}\left[\begin{array}{l}
\boldsymbol{y}-\boldsymbol{y}_{e} \\
\theta-\theta_{e}
\end{array}\right], \boldsymbol{p}_{k}\right\rangle f_{k}\right) .
\end{array}\right.
$$

Let $d=2,3, q>d, 1<p<\frac{2 q}{2 q-1}$. If $\rho>0$ is sufficiently small, then

(i) the feedback problem (2.24) admits a unique (fixed point nonlinear semigroup) solution

$$
\begin{gathered}
\{\boldsymbol{y}, \theta\} \in \boldsymbol{X}_{p, q, \sigma}^{\infty} \times X_{p, q}^{\infty} \equiv L^{p}\left(0, \infty ; \mathcal{D}\left(\mathbb{A}_{F, q}\right)\right) \cap W^{1, p}\left(0, \infty ; \boldsymbol{W}_{\sigma}^{q}(\Omega)\right) \\
\boldsymbol{X}_{p, q, \sigma}^{\infty} \times X_{p, q}^{\infty} \hookrightarrow C\left([0, \infty] ; \boldsymbol{V}_{b}^{q, p}(\Omega)\right)
\end{gathered}
$$

as in (2.18);

(ii) there exists $\widetilde{\gamma}>0, M_{\widetilde{\gamma}}>0$ such that said solution $\{\boldsymbol{y}, \theta\}$ satisfies

$$
\left\|\left[\begin{array}{c}
\boldsymbol{y}(t)-\boldsymbol{y}_{e} \\
\theta(t)-\theta_{e}
\end{array}\right]\right\|_{\boldsymbol{V}_{b}^{q, p}(\Omega)} \leq M_{\widetilde{\gamma}} e^{-\widetilde{\gamma} t}\left\|\left[\begin{array}{c}
\boldsymbol{y}_{0}-\boldsymbol{y}_{e} \\
\theta_{0}-\theta_{e}
\end{array}\right]\right\|_{\boldsymbol{V}_{b}^{q, p}(\Omega)}, \quad t \geq 0 . \square
$$

\subsection{Comparison with the literature.}

1. The critical feature - the ignition key - to get the analysis of the present paper succeed: an inverse theory problem of the Unique Continuation Property type, which is established via Carleman-type estimates 89 . These tools are used in the preliminary Section 4, dealing with the finite dimensional unstable projected problem (3.6a) at the level of establishing the essential Kalman controllability condition (4.31). This ultimately rests on the UCP reported in Appendix B, one of the several UCPs proved in [89], by means of Carleman-type inequalities. The original Carleman estimates (characterized by a suitable exponential weight) were introduced in [17] in 1939 to establish uniqueness of a PDE on two variables. A historical account is given in [50, Section 1.1, pp2-3] from which we quote: "In 1979, the growing interest to global uniqueness results for multidimensional CIPs with the lateral data stimulated Klibanov to apply the Carleman estimates for establishing such results. At the same time, a similar global uniqueness theorem was independently formulated and proven by Bukhgeim. As a result, the method of Carleman estimates was presented to the inverse problem community in the joint paper [14]. The detailed proofs were published in [13] and [45]". See also [46], 47] for early literature and [49] for a hyperbolic paper. The authors of the present paper are most pleased to offer a contribution to the special volume of JIIP to recognize the pioneering workand subsequent intensive production-of M. V. Klibanov on Carleman estimates and inverse theory. For a discussion on Carleman-type inequalities in integral form with lower-order terms and pointwise Carleman estimates with no lower order terms, we refer to [63] and to [59, Section 1.5]. A recent book on these topics is 38.

2. The Besov space setting. With reference to both the "Motivation" of Subsection 1.1 as well as Subsection 1.3, it was already emphasized that all prior literature on the problem of feedback stabilization of either the Navier-Stokes equations or, subsequently, the Boussinesq system is carried out in a HilbertSobolev setting. As already noted, this treatment is inadequate to obtain finite dimensionality in full generality of the localized tangential boundary feedback control for the 3d-Navier-Stokes equations. This obstacle then motivated the introduction of the $\boldsymbol{L}^{q}$-Sobolev/Besov setting in 60, 62] with tight indices, see (1.4), (1.11), that does not recognize boundary conditions, see Remark [1.2] and subsequent orientation, in order to solve affirmatively such open problem on the finite dimensionality 
in 3d-Navier-Stokes tangential boundary feedback stabilization. Reference [60] on localized interior controls sets the preparatory stage for the more demanding boundary control case in 62.

3. The Boussinesq system. As already noted in the "Motivation" of Subsection 1.1 the present paper is the first contribution in the Besov setting toward the uniform stabilization of the Boussinesq system with a localized boundary stabilizing feedback control (finite dimensional): namely, the Dirichlet boundary control for the heat component acting on $\widetilde{\Gamma}$. It follows the preliminary test case with two stabilizing feedback controls - one for the fluid component and one for the heat component - localized on an arbitrary small set $\omega \subset \Omega$. In turn, the present paper sets the stage for attacking our final goal: uniform stabilization of the Boussinesq system by means of a localized boundary, finite dimensional feedback stabilizing control acting this time on the Navier-Stokes component, of the same type as in the case of the N-S alone 62. The topic of stabilization (by open loop controls) of Navier-Stokes equations originated in the pioneering work of [28].

4. Review of the literature on the feedback stabilization of the Boussinesq system. The first contribution is due to 92 via internal feedback controls in the Hilbert setting $\boldsymbol{H} \times L^{2}(\Omega)$, where $\boldsymbol{H}$ is the usual Hilbert $\boldsymbol{L}_{\sigma}^{2}(\Omega)$-specialization of (1.5) [19]. The feedback controllers are both infinite-dimensional

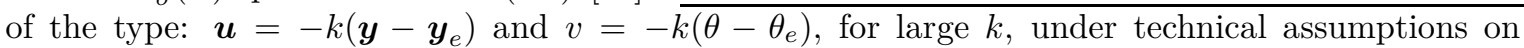
the localization of the controls. Paper 64] also studies the feedback stabilization problem with, eventually, localized controls, which again are infinite dimensional; eg defined in terms of a subdifferential. These results are in stark contrast with the present work. Here - first, we establish that the stabilizing control is finite dimensional; and, second, we show that its fluid component is of reduced dimension, as expressed only by means of $(d-1)$-components to include necessarily the $d^{\text {th }}$ component. Finally paper [71] studies the feedback stabilization problem with, this time, mixed boundary and claims to provide a rigorous treatment of problems studied in [15, [16.

5. It is as a by-product of the described UCP, as applied to the adjoint static problem rather than the original static problem, that we can extract the further benefit of obtaining the closed-loop feedback control acting on the fluid component of reduced dimension: $(d-1)$ rather than $d$. There is however a key difference with respect to a similar property in 61] with two localized internal controls. Namely, in 61 the $d^{\text {th }}$ component of the fluid control is not needed; in the present case, it is essential. This dimension reduction in the closed-loop feedback stabilizing fluid control is in line with the open-loop controllability results in 20, 21. No such reduction of components is present in the case of uniform stabilization of the Navier-Stokes equations per-se.

6. Geometric Multiplicity. We also point out that, as in [56], [57] and [60, 62 for the Navier-Stokes equations, in the case of the Boussinesq system, the number of needed controls will be related to the more desirable geometric multiplicity, not the larger algebraic multiplicity as in prior treatments, [9, [7, p 276] of the unstable eigenvalues, by using the classical test for controllability of a system in Jordan form [18, [8, p 464]. This allows one also to obtain constructively an explicit form of the finite dimensional feedback control; and, moreover, to show that the feedback control acting on the fluid may be taken of reduced dimension: one unit less, i.e. $d-1$, than the fluid component of dimension $d$. As noted in point 5. above, this is due to the Unique Continuation Property of the adjoint problem, reported in Appendix B It represents an additional contribution of the present paper.

7. Maximal regularity of the linearized feedback problem. In the present contribution, following 60, [61, 62], the analysis of the original non-linear problem is carried out in the context of the property of maximal regularity of the linearized feedback $\boldsymbol{w}$-problem (5.3a); that is, of the operator $\mathbb{A}_{F, q}$ in (5.3a)-(5.5b). This applies to both well-posedness (in Section 8) as well as stabilization (in Section 9) of the nonlinear feedback problem. This is in contrast with prior treatments, such as [9, [7, which often rely on chopping the nonlinearity and carrying out a limit process. The maximal regularity approach introduced for stabilization problems in 60 is cleaner and more desirable both technically and conceptually. On the other hand, applicability of Maximal Regularity requires well balanced spaces. Recent developments in this particular area [93, 75, [69, [53, 54], 23] etc. were critical for carrying out our analysis. 


\section{Beginning with the proof of Theorem 2.1, Spectral decompo- sition of the linearized $w$-problem (1.31) or (1.32).}

We return to the assumed starting point of the present paper, which is that the free (uncontrolled) dynamics operator $\mathbb{A}_{q}$ in (1.34) is unstable, see (1.38). Its properties are collected in Theorem 1.2. Accordingly, its eigenvalues satisfy the statement which includes their location in (1.38). Denote by $P_{N}$ and $P_{N}^{*}$ (which actually depend on $q$ ) the projections given explicitly by [40, p 178], [9], 10]

$$
\begin{aligned}
& P_{N}=-\frac{1}{2 \pi i} \int_{\boldsymbol{\Gamma}}\left(\lambda I-\mathbb{A}_{q}\right)^{-1} d \lambda: \boldsymbol{W}_{\sigma}^{q} \text { onto }\left(\boldsymbol{W}_{\sigma}^{q}\right)_{N}^{u} \subset \boldsymbol{L}_{\sigma}^{q}(\Omega) \times L^{q}(\Omega) \\
& P_{N}^{*}=-\frac{1}{2 \pi i} \int_{\overline{\boldsymbol{\Gamma}}}\left(\lambda I-\mathbb{A}_{q}^{*}\right)^{-1} d \lambda:\left(\boldsymbol{W}_{\sigma}^{q}\right)^{*} \text { onto }\left[\left(\boldsymbol{W}_{\sigma}^{q}\right)_{N}^{u}\right]^{*} \subset \boldsymbol{L}_{\sigma}^{q^{\prime}}(\Omega) \times L^{q^{\prime}}(\Omega) .
\end{aligned}
$$

Here $\boldsymbol{\Gamma}$ (respectively, its conjugate counterpart $\overline{\boldsymbol{\Gamma}}$ ) is a smooth closed curve that separates the unstable spectrum from the stable spectrum of $\mathbb{A}_{q}$ (respectively, $\mathbb{A}_{q}^{*}$ ). As in [10, Sect 3.4, p 37], following [84, [85], we decompose the space $\boldsymbol{W}_{\sigma}^{q}=\boldsymbol{W}_{\sigma}^{q}(\Omega) \equiv \boldsymbol{L}_{\sigma}^{q}(\Omega) \times L^{q}(\Omega)$ into the sum of two complementary subspaces (not necessarily orthogonal):

$$
\boldsymbol{W}_{\sigma}^{q}=\left(\boldsymbol{W}_{\sigma}^{q}\right)_{N}^{u} \oplus\left(\boldsymbol{W}_{\sigma}^{q}\right)_{N}^{s} ;\left(\boldsymbol{W}_{\sigma}^{q}\right)_{N}^{u} \equiv P_{N} \boldsymbol{W}_{\sigma}^{q} ;\left(\boldsymbol{W}_{\sigma}^{q}\right)_{N}^{s} \equiv\left(I-P_{N}\right) \boldsymbol{W}_{\sigma}^{q} ;
$$

$$
\operatorname{dim}\left(\boldsymbol{W}_{\sigma}^{q}\right)_{N}^{u}=N
$$

where each of the spaces $\left(\boldsymbol{W}_{\sigma}^{q}\right)_{N}^{u}$ and $\left(\boldsymbol{W}_{\sigma}^{q}\right)_{N}^{s}$ (which depend on $q$, but we suppress such dependence) is invariant under $\mathbb{A}_{q}$, and let

$$
\mathbb{A}_{q, N}^{u}=P_{N} \mathbb{A}_{q}=\left.\mathbb{A}_{q}\right|_{\left(\boldsymbol{W}_{\sigma}^{q}\right)_{N}^{u}} ; \quad \mathbb{A}_{q, N}^{s}=\left(I-P_{N}\right) \mathbb{A}_{q}=\left.\mathbb{A}_{q}\right|_{\left(\boldsymbol{W}_{\sigma}^{q}\right)_{N}^{s}}
$$

be the restrictions of $\mathbb{A}_{q}$ to $\left(\boldsymbol{W}_{\sigma}^{q}\right)_{N}^{u}$ and $\left(\boldsymbol{W}_{\sigma}^{q}\right)_{N}^{s}$ respectively. The original point spectrum (eigenvalues) $\left\{\lambda_{j}\right\}_{j=1}^{\infty}$ of $\mathbb{A}_{q}$ is then split into two sets, recall (1.38)

$$
\sigma\left(\mathbb{A}_{q, N}^{u}\right)=\left\{\lambda_{j}\right\}_{j=1}^{N} ; \quad \sigma\left(\mathbb{A}_{q, N}^{s}\right)=\left\{\lambda_{j}\right\}_{j=N+1}^{\infty},
$$

and $\left(\boldsymbol{W}_{\sigma}^{q}\right)_{N}^{u}$ is the generalized eigenspace of $\mathbb{A}_{q, N}^{u}$ in (3.3). The system (1.32) on $\boldsymbol{W}_{\sigma}^{q} \equiv \boldsymbol{L}_{\sigma}^{q}(\Omega) \times L^{q}(\Omega)$ can accordingly be decomposed as

$$
\boldsymbol{w}=\boldsymbol{w}_{N}+\boldsymbol{\zeta}_{N}, \quad \boldsymbol{w}_{N}=P_{N} \boldsymbol{w}, \quad \boldsymbol{\zeta}_{N}=\left(I-P_{N}\right) \boldsymbol{w} .
$$

After applying $P_{N}$ and $\left(I-P_{N}\right)$ (which commute with $\left.\mathbb{A}_{q}\right)$ on (1.32), we obtain via (3.3)

$$
\begin{gathered}
\text { on }\left(\boldsymbol{W}_{\sigma}^{q}\right)_{N}^{u}: \boldsymbol{w}_{N}^{\prime}-\mathbb{A}_{q, N}^{u} \boldsymbol{w}_{N}=P_{N}\left[\begin{array}{c}
P_{q}(m \boldsymbol{u}) \\
\mathcal{B}_{q} D v
\end{array}\right] ; \quad \boldsymbol{w}_{N}(0)=P_{N}\left[\begin{array}{c}
\boldsymbol{w}_{f}(0) \\
w_{h}(0)
\end{array}\right] \\
\text { on }\left(\boldsymbol{W}_{\sigma}^{q}\right)_{N}^{s}: \boldsymbol{\zeta}_{N}^{\prime}-\mathbb{A}_{q, N}^{s} \boldsymbol{\zeta}_{N}=\left(I-P_{N}\right)\left[\begin{array}{c}
P_{q}(m \boldsymbol{u}) \\
\mathcal{B}_{q} D v
\end{array}\right] ; \quad \boldsymbol{\zeta}_{N}(0)=\left(I-P_{N}\right)\left[\begin{array}{l}
\boldsymbol{w}_{f}(0) \\
w_{h}(0)
\end{array}\right]
\end{gathered}
$$

respectively, where $\boldsymbol{w}_{N}=\left\{\boldsymbol{w}_{f, N}, w_{h, N}\right\}, \boldsymbol{\zeta}_{N}=\left\{\boldsymbol{\zeta}_{f, N}, \zeta_{h, N}\right\}$. See [10, Appendix A] for the extension of $P_{N}$ outside $\boldsymbol{W}_{\sigma}^{q}$. 


\section{Proof of Theorem 2.1. Global well-posedness and uniform exponential stabilization of the linearized $w$-problem (1.32) on the space $\boldsymbol{W}_{\sigma}^{q}(\Omega) \equiv \boldsymbol{L}_{\sigma}^{q}(\Omega) \times L^{q}(\Omega)$ or the space $\boldsymbol{V}_{b}^{q, p}(\Omega) \equiv \widetilde{\boldsymbol{B}}_{q, p}^{2-2 / p}(\Omega) \times$ $B_{q, p}^{2-2 / p}(\Omega)$.}

\subsection{Orientation.}

We shall appeal to several technical developments in [60], where the case of the N-S equations has been studied. We will have to adopt and transfer some of its procedures to the case of the Boussinesq system. Thus, properties such as maximal regularity and the entire development for uniform stabilization of the linearized Boussinesq dynamics need to be established. We have already noted that while analyticity and maximal regularity are equivalent properties in the Hilbert setting [22] this is not so in the Banach setting where maximal regularity is a more general and more delicate property. Moreover, it is only after we establish uniform stabilization that we can claim maximal regularity up to infinity. This needs to be asserted by direct analysis. To proceed, we recall the state space $\boldsymbol{W}_{\sigma}^{q}(\Omega)=\boldsymbol{L}_{\sigma}^{q}(\Omega) \times L^{q}(\Omega) \equiv\left(\boldsymbol{W}_{\sigma}^{q}\right)_{N}^{u} \oplus\left(\boldsymbol{W}_{\sigma}^{q}\right)_{N}^{s}$ in (1.34), (3.2). The unstable uncontrolled operator is $\mathbb{A}_{q}$ in (1.34).

The same strategy employed in [60] seeks to show that the linearized $\boldsymbol{w}$-dynamics (2.4) in feedback form that is, the operator $\mathbb{A}_{F, q}$ in (2.5), (2.6b) - of the translated non-linear $\{\boldsymbol{z}, h\}$-problem is uniformly stable in the desired setting in terms of finite dimensional feedback controls. Here $\boldsymbol{w}=\left\{\boldsymbol{w}_{f}, w_{h}\right\}$ comprises the $d$-dimensional fluid component $\boldsymbol{w}_{f}$ and scalar heat component $w_{h}$. To this end, we first seek to establish that the finite dimensional projection - the $\boldsymbol{w}_{N}$-equation in (3.6a) is controllable on $\left(\boldsymbol{W}_{\sigma}^{q}\right)_{N}^{u}$, hence exponentially stabilizable with an arbitrarily large decay rate [94, p. 44]. This is done in Theorem 4.1 (controllability) and Theorem 4.2 (stabilization). Once it is established (Theorem 6.1) that the operator $\mathbb{A}_{F, q}$ generates a s.c. analytic semigroup, the next step is to examine the corresponding $\boldsymbol{\zeta}_{N^{-}}$-equation, (3.6b), where the arbitrarily large decay rate of the feedback $\boldsymbol{w}_{N}$-equation combined with the exponential stability on $\left(\boldsymbol{W}_{\sigma}^{q}\right)_{N}^{s}$ of the s.c. analytic semigroup $e^{\mathbb{A}_{q, N}^{s} t}$ (whose spectrum is on the left of $R e \lambda_{N+1}<0$ ), yields the desired result. This is Theorem 6.2. One needs to emphasize that all this works thanks to the present corresponding Unique Continuation Property, this time of the Boussinesq system, that is Theorem B.1 in Appendix B. It is this UCP that permits one to verify the Kalman algebraic condition (4.31) of Theorem 4.1. This is established asserting the Claim in the proof of Theorem 4.1 Such condition is equivalent to linear independence of certain vectors occurring in (4.31) below, so that the finite dimensional projected $\boldsymbol{w}_{N}$-dynamics satisfies the controllability condition of Kalman or Hautus. See corresponding cases in [86], 87]. The proof of Theorem 4.1] invokes all $d$-components of the fluid vectors $\boldsymbol{u}_{k}$. However, one can do better. The UCP of Theorem B.1. Appendix B does not include boundary conditions. On the other hand, in our present case, the UCP is applied to the adjoint eigenproblem (4.3) below, which of course includes also homogeneous B. C. such as (4.10d). It then turns out that by taking advantage also of these homogeneous B. C. (4.10d), it is possible through a proof of a UCP given in 89, Theorem 6, Theorem 7] to obtain an improvement whereby the new version of Theorem 4.1 (verification of the Kalman algebraic condition (4.31) ) involves only $(d-1)$-components of the $d$-vector $\boldsymbol{u}_{k}$, to include necessarily the $d^{\text {th }}$ component. Such improvement is given in Appendix C. The proof uses a variation of the proof of the UCP [89, Theorem 6 or 7]. This result is in contrast with two other cases. First, the uniform stabilization study of the Boussinesq system by localized interior feedback controls for both fluid and heat components (acting on an arbitrarily small internal set $\omega \subset \Omega$ ) 61, again $(d-1)$-components of the fluid vector $\boldsymbol{u}_{k}$ are involved, but this time, it is the $d^{\text {th }}$ component that may be omitted, in contrast with the case of the present paper. Second, in the cases of uniform stabilization of the NavierStokes equations, either by internal localized [60] or boundary localized controls [62, all $d$-components of the fluid vector $\boldsymbol{u}_{k}$ are needed. The explanation is in the nature of the Boussinesq adjoint eigenprob- 
lem. Henceforth the conceptual advantage of having our results invoking only $(d-1)$-components of the fluid vectors $\boldsymbol{u}_{k}$, including the $d^{\text {th }}$ component will be responsible for heavier notation as described below.

For each $i=1, \ldots, M$, we denote by $\left\{\boldsymbol{\Phi}_{i j}\right\}_{j=1}^{\ell_{i}},\left\{\boldsymbol{\Phi}_{i j}^{*}\right\}_{j=1}^{\ell_{i}}$ the normalized, linearly independent eigenfunctions of $\mathbb{A}_{q}$, respectively $\mathbb{A}_{q}^{*}$, say, on

$$
\begin{aligned}
& \boldsymbol{W}_{\sigma}^{q}(\Omega) \equiv \boldsymbol{L}_{\sigma}^{q}(\Omega) \times L^{q}(\Omega) \text { and } \\
& \qquad\left(\boldsymbol{W}_{\sigma}^{q}(\Omega)\right)^{*} \equiv\left(\boldsymbol{L}_{\sigma}^{q}(\Omega)\right)^{\prime} \times\left(L^{q}(\Omega)\right)^{\prime}=\boldsymbol{L}_{\sigma}^{q^{\prime}}(\Omega) \times L^{q^{\prime}}(\Omega), \quad \frac{1}{q}+\frac{1}{q^{\prime}}=1,
\end{aligned}
$$

(where in the last equality we have invoked the identity below (1.6)) corresponding to the $M$ distinct unstable eigenvalues $\lambda_{1}, \ldots, \lambda_{M}$ of $\mathbb{A}_{q}$ and $\bar{\lambda}_{1}, \ldots, \bar{\lambda}_{M}$ of $\mathbb{A}_{q}^{*}$ respectively, either on $\boldsymbol{W}_{\sigma}^{q}(\Omega)$ or on $\boldsymbol{V}^{q, p}(\Omega)$ :

$$
\begin{aligned}
& \mathbb{A}_{q} \boldsymbol{\Phi}_{i j}=\lambda_{i} \boldsymbol{\Phi}_{i j} \in \mathcal{D}\left(\mathbb{A}_{q}\right)=\left[\boldsymbol{W}^{2, q}(\Omega) \cap \boldsymbol{W}_{0}^{1, q}(\Omega) \cap \boldsymbol{L}_{\sigma}^{q}(\Omega)\right] \times\left[W^{2, q}(\Omega) \cap W_{0}^{1, q}(\Omega)\right] \\
& \mathbb{A}_{q}^{*} \boldsymbol{\Phi}_{i j}^{*}=\bar{\lambda}_{i} \boldsymbol{\Phi}_{i j}^{*} \in \mathcal{D}\left(\mathbb{A}_{q}^{*}\right)=\left[\boldsymbol{W}^{2, q^{\prime}}(\Omega) \cap \boldsymbol{W}_{0}^{1, q^{\prime}}(\Omega) \cap \boldsymbol{L}_{\sigma}^{q^{\prime}}(\Omega)\right] \times\left[W^{2, q^{\prime}}(\Omega) \cap W_{0}^{1, q^{\prime}}(\Omega)\right] .
\end{aligned}
$$

It is the adjoint problem (4.3) which is of our interest:

\subsection{The adjoint operator $\mathbb{A}_{q}^{*}$ of $\mathbb{A}_{q}$ in (1.34).}

It is computed in 61] that the adjoint $\mathbb{A}_{q}^{*}$ of $\mathbb{A}_{q}$ is given by

$$
\begin{aligned}
\mathbb{A}_{q}^{*}=\left[\begin{array}{cc}
\mathcal{A}_{q}^{*} & -\mathcal{C}_{\theta_{e}}^{*} \\
-\mathcal{C}_{\gamma}^{*} & \mathcal{B}_{q}^{*}
\end{array}\right] & : \boldsymbol{W}_{\sigma}^{q^{\prime}}(\Omega)=\boldsymbol{L}_{\sigma}^{q^{\prime}}(\Omega) \times L^{q}(\Omega) \supset \mathcal{D}\left(\mathbb{A}_{q}^{*}\right)=\mathcal{D}\left(\mathcal{A}_{q}^{*}\right) \times \mathcal{D}\left(\mathcal{B}_{q}^{*}\right) \\
& =\left(\boldsymbol{W}^{2, q^{\prime}}(\Omega) \cap \boldsymbol{W}_{0}^{1, q^{\prime}}(\Omega) \cap \boldsymbol{L}_{\sigma}^{q^{\prime}}(\Omega)\right) \times\left(W^{2, q^{\prime}}(\Omega) \cap W_{0}^{1, q^{\prime}}(\Omega)\right) \longrightarrow \boldsymbol{W}_{\sigma}^{q^{\prime}}(\Omega) .
\end{aligned}
$$

where the adjoints $\mathcal{C}_{\theta_{e}}^{*}$ and $\mathcal{C}_{\gamma}^{*}$ of the operators $\mathcal{C}_{\theta_{e}}$ and $\mathcal{C}_{\gamma}$ in (1.21), (1.22) are

$$
\begin{gathered}
\mathcal{C}_{\theta_{e}}^{*} \psi^{*}=P_{q^{\prime}}\left(\psi^{*} \nabla \theta_{e}\right) \in \boldsymbol{L}_{\sigma}^{q^{\prime}}(\Omega), \quad \mathcal{C}_{\gamma}^{*} \boldsymbol{\varphi}^{*}=-\gamma\left(P_{q^{\prime}} \varphi^{*}\right) \cdot \boldsymbol{e}_{d}, \\
\mathcal{C}_{\gamma}^{*} \in \mathcal{L}\left(\boldsymbol{L}_{\sigma}^{q^{\prime}}(\Omega), L^{q^{\prime}}(\Omega)\right) . \\
\mathcal{A}_{q}^{*}=-\left(\nu A_{q}^{*}+A_{o, q}^{*}\right), A_{q}^{*} \boldsymbol{f}=-P_{q^{\prime}} \Delta \boldsymbol{f}, \\
\mathcal{D}\left(A_{q}^{*}\right) \equiv \boldsymbol{W}^{2, q^{\prime}}(\Omega) \cap \boldsymbol{W}_{0}^{1, q^{\prime}}(\Omega) \cap \boldsymbol{L}_{\sigma}^{q^{\prime}}(\Omega) \\
\left(A_{o . q}\right)^{*}=A_{o, q}^{*}=P_{q^{\prime}}\left(L_{e}\right)^{*}: \boldsymbol{W}^{1, q^{\prime}}(\Omega) \longrightarrow \boldsymbol{L}_{\sigma}^{q^{\prime}}(\Omega) \\
\mathcal{B}_{q}^{*} \psi=-\kappa \Delta \psi-\boldsymbol{y}_{e} \cdot \nabla \psi, \mathcal{D}\left(\mathcal{B}_{q}^{*}\right)=W^{2, q^{\prime}}(\Omega) \cap W_{0}^{1, q^{\prime}}(\Omega) .
\end{gathered}
$$

The expression in (4.8) is obtained from (1.18) integration by parts using $\left.\boldsymbol{y}_{e}\right|_{\Gamma}=0$ and $\operatorname{div} \boldsymbol{y}_{e}=0$ from (1.2c-d). See Appendix $\mathbb{B}$ Notice that in passing from $\mathbb{A}_{q}$ to $\mathbb{A}_{q}^{*}$ the operators $\mathcal{C}_{\gamma}$ and $\mathcal{C}_{\theta_{e}}$ switch places with their adjoints. This fact has a key implication on the needed Unique Continuation Property. With $\boldsymbol{\Phi}^{*}=\left[\boldsymbol{\varphi}^{*}, \psi^{*}\right]$, the explicit version of $\mathbb{A}_{q}^{*} \boldsymbol{\Phi}=\lambda \boldsymbol{\Phi}^{*}$ is by (4.4)

$$
\left\{\begin{aligned}
\mathcal{A}_{q}^{*} \varphi^{*}-\mathcal{C}_{\theta_{e}}^{*} \psi^{*} & =\lambda \varphi^{*} \\
-\mathcal{B}_{q}^{*} \psi^{*}-\mathcal{C}_{\gamma}^{*} \varphi^{*} & =\lambda \psi^{*}
\end{aligned}\right.
$$

while its PDE-version is, by virtue of (4.4)-(4.8)

$$
\left\{\begin{aligned}
-\nu \Delta \boldsymbol{\varphi}^{*}+L_{e}^{*}\left(\boldsymbol{\varphi}^{*}\right)+\psi^{*} \nabla \theta_{e}+\nabla \pi & =\lambda \boldsymbol{\varphi}^{*} & & \text { in } \Omega \\
-\kappa \Delta \psi^{*}-\boldsymbol{y}_{e} \cdot \nabla \psi^{*}-\gamma \boldsymbol{\varphi}^{*} \cdot \boldsymbol{e}_{d} & =\lambda \psi^{*} & & \text { in } \Omega \\
\operatorname{div} \boldsymbol{\varphi}^{*} & =0 & & \text { in } \Omega \\
\boldsymbol{\varphi}^{*}=0, \psi^{*} & =0 & & \text { on } \Gamma .
\end{aligned}\right.
$$




\subsection{Critical step: uniform stabilization of the unstable finite dimensional $\boldsymbol{w}_{N}$-projection (3.6a) by feedback controls $\{v, \boldsymbol{u}\}$.}

For simplicity and space constraints, we provide here explicitly only the conceptually and computationally more amenable case, where the operator $\mathbb{A}_{q, N}^{u}$ in (3.3) is semisimple [40] on the space $\left(\boldsymbol{W}_{\sigma}^{q}\right)_{N}^{u}$ of dimension $N=\ell_{1}+\cdots+\ell_{M}$. How to treat the general case is known [56, [57, 60], 62]. It is much more computationally intensive.

Assumption: Thus assume henceforth that

$$
\mathbb{A}_{q, N}^{u} \text { is semisimple on }\left(\boldsymbol{W}_{\sigma}^{q}\right)_{N}^{u} \text { : }
$$

that is, for the unstable eigenvalues $\lambda_{1}, \ldots, \lambda_{M}$ geometric and algebraic multiplicity coincide.

Accordingly, let $\boldsymbol{w} \in\left(\boldsymbol{W}_{\sigma}^{q}\right)_{N}^{u}$, then [40, p51]

$$
\left(\boldsymbol{W}_{\sigma}^{q}\right)_{N}^{u} \ni \boldsymbol{w}_{N}=\sum_{i, j=1}^{M, \ell_{i}}\left\langle\boldsymbol{w}_{N}, \boldsymbol{\Phi}_{i j}^{*}\right\rangle_{\boldsymbol{W}_{\sigma}^{q},\left(\boldsymbol{W}_{\sigma}^{q}\right)^{*}} \boldsymbol{\Phi}_{i j}, \boldsymbol{\Phi}_{i j}=\left[\begin{array}{c}
\boldsymbol{\varphi}_{\boldsymbol{i j}} \\
\psi_{i j}
\end{array}\right] .
$$

Define $\beta_{i}$ and $\beta$ the following ordered bases of length $\ell_{i}$ and $N$, respectively, corresponding to the unstable eigenvalues

$$
\begin{aligned}
\beta_{i} & =\left[\boldsymbol{\Phi}_{i 1}, \ldots, \mathbf{\Phi}_{i \ell_{i}}\right], i=1, \ldots, M \\
\beta & =\beta_{1} \cup \beta_{2} \cup \cdots \cup \beta_{M}=\left[\mathbf{\Phi}_{11}, \ldots, \boldsymbol{\Phi}_{1 \ell_{1}}, \mathbf{\Phi}_{21}, \ldots, \mathbf{\Phi}_{2 \ell_{2}}, \ldots, \mathbf{\Phi}_{M 1}, \ldots, \mathbf{\Phi}_{M \ell_{M}}\right] .
\end{aligned}
$$

Thus the matrix representation of the operator $\mathbb{A}_{q, N}^{u}$ in (3.6a) on $\left(\boldsymbol{W}_{\sigma}^{q}\right)_{N}^{u}$ with respect to the basis $\beta$ is

$$
\left[\mathbb{A}_{q, N}^{u}\right]_{\beta}=\Lambda=\left[\begin{array}{cccc}
\lambda_{1} I_{1} & & & 0 \\
& \lambda_{2} I_{2} & & \\
& & \ddots & \\
0 & & & \lambda_{M} I_{M}
\end{array}\right]: N \times N, I_{i}: \ell_{i} \times \ell_{i} .
$$

Next, with reference to (3.6a) with $m \boldsymbol{u} \in \boldsymbol{L}^{q}(\omega), P_{q}(m \boldsymbol{u}) \in \boldsymbol{L}_{\sigma}^{q}(\omega)$ and $v \in L^{q}(\Gamma)$, we shall show that:

$$
\begin{aligned}
P_{N}\left[\begin{array}{c}
P_{q}(m \boldsymbol{u}) \\
\mathcal{B}_{q} D v
\end{array}\right] & =\sum_{i, j=1}^{M, \ell_{i}}\left\langle P_{N}\left[\begin{array}{c}
P_{q}(m \boldsymbol{u}) \\
\mathcal{B}_{q} D v
\end{array}\right], \boldsymbol{\Phi}_{\boldsymbol{i j}}^{*}\right\rangle_{\boldsymbol{W}_{\sigma}^{q},\left(\boldsymbol{W}_{\sigma}^{q}\right)^{*}} \boldsymbol{\Phi}_{i j} \\
& =\sum_{i, j=1}^{M, \ell_{i}}\left[\left\langle m \boldsymbol{u}, \boldsymbol{\varphi}_{i j}^{*}\right\rangle_{\boldsymbol{L}_{\sigma}^{q}(\omega), \boldsymbol{L}_{\sigma}^{q^{\prime}}(\omega)}-\left\langle v,\left.\frac{\partial \psi_{i j}^{*}}{\partial \nu}\right|_{\Gamma}\right\rangle_{L^{q}(\Gamma), L^{q^{\prime}}(\Gamma)}\right] \boldsymbol{\Phi}_{i j}
\end{aligned}
$$

In fact, since $P_{N}^{*} \boldsymbol{\Phi}_{i j}^{*}=\boldsymbol{\Phi}_{i j}^{*}$ and $P_{q^{\prime}} \boldsymbol{\varphi}_{i j}^{*}=\boldsymbol{\varphi}_{i j}^{*}$, we obtain from (4.15), recalling $\boldsymbol{W}_{\sigma}^{q}$ from (1.34), $\boldsymbol{\Phi}^{*}=$ $\left[\varphi_{i j}^{*}, \psi_{i j}^{*}\right]:$

$$
\begin{aligned}
P_{N}\left[\begin{array}{c}
P_{q}(m \boldsymbol{u}) \\
\mathcal{B}_{q} D v
\end{array}\right] & =\sum_{i, j=1}^{M, \ell_{i}}\left[\left\langle P_{q}(m \boldsymbol{u}), \boldsymbol{\varphi}_{i j}^{*}\right\rangle_{\boldsymbol{L}_{\sigma}^{q}(\omega), \boldsymbol{L}_{\sigma}^{q^{\prime}(\omega)}}+\left\langle v, D^{*} \mathcal{B}_{q}^{*} \psi_{i j}^{*}\right\rangle_{L^{q}(\Gamma), L^{q^{\prime}}(\Gamma)}\right] \boldsymbol{\Phi}_{i j} \\
& =\sum_{i, j=1}^{M, \ell_{i}}\left[\left\langle m \boldsymbol{u}, \boldsymbol{\varphi}_{i j}^{*}\right\rangle_{\boldsymbol{L}_{\sigma}^{q}(\omega), \boldsymbol{L}_{\sigma}^{q^{\prime}}(\omega)}+\left\langle v,-\left.\frac{\partial \psi_{i j}^{*}}{\partial \nu}\right|_{\Gamma}\right\rangle_{L^{q}(\Gamma), L^{q^{\prime}}(\Gamma)}\right] \boldsymbol{\Phi}_{i j}
\end{aligned}
$$


and (4.16) is verified. In going from (4.17) to (4.18) we have recalled $D^{*} \mathcal{B}_{q}^{*} f=-\frac{\partial f}{\partial \nu}, f \in \mathcal{D}\left(\mathcal{B}_{q}^{*}\right)$, see (B.11) of Appendix B for $\kappa=1$. Next, we seek an interior control $\boldsymbol{u}$ acting on $\omega$ and a boundary control $v$ acting on $\widetilde{\Gamma}$ at first of the form

$$
\begin{aligned}
& \boldsymbol{u}=\sum_{k=1}^{K} \mu_{k}(t) \boldsymbol{u}_{k}, \boldsymbol{u}_{k} \in\left(\boldsymbol{W}_{\sigma}^{q}\right)_{N}^{u} \subset \boldsymbol{L}_{\sigma}^{q}(\Omega) \\
& v=\sum_{k=1}^{K} \nu_{k}(t) f_{k} \in \mathcal{F} \subset W^{2-1 /{ }^{1}, q}(\widetilde{\Gamma})
\end{aligned}
$$

where

$$
\mathcal{F}=\operatorname{span}\left\{\left.\frac{\partial \psi_{i j}^{*}}{\partial \nu}\right|_{\widetilde{\Gamma}}, i=1, \ldots, M ; j=1, \ldots, \ell_{i}\right\} \subset W^{2-1 / q, q}(\widetilde{\Gamma}) .
$$

Thus substituting (4.19), (4.20), in (4.16) we obtain

$$
\begin{aligned}
P_{N}\left[\begin{array}{c}
P_{q}(m \boldsymbol{u}) \\
\mathcal{B}_{q} D v
\end{array}\right]=\sum_{i, j=1}^{M, \ell_{i}} \sum_{k=1}^{K}\left[\left\langle m \boldsymbol{u}_{k}, \boldsymbol{\varphi}_{i j}^{*}\right\rangle_{\boldsymbol{L}_{\sigma}^{q}(\omega), \boldsymbol{L}_{\sigma}^{q^{\prime}(\omega)}} \mu_{k}(t)\right. & \\
& \left.-\left\langle f_{k},\left.\frac{\partial \psi_{i j}^{*}}{\partial \nu}\right|_{\Gamma}\right\rangle_{L^{q}(\Gamma), L^{q^{\prime}}(\Gamma)} \nu_{k}(t)\right] \boldsymbol{\Phi}_{i j} .
\end{aligned}
$$

Accordingly, in view of (4.22), we can rewrite the $\boldsymbol{w}_{N}$-problem (3.6a) as

$$
\text { on } \begin{aligned}
\left(\boldsymbol{W}_{\sigma}^{q}\right)_{N}^{u}: \boldsymbol{w}_{N}^{\prime}=\mathbb{A}_{q, N}^{u} \boldsymbol{w}_{N}+\sum_{i, j=1}^{M, \ell_{i}} \sum_{k=1}^{K}\left[\left\langle m \boldsymbol{u}_{k}, \boldsymbol{\varphi}_{i j}^{*}\right\rangle_{\boldsymbol{L}_{\sigma}^{q}(\omega), \boldsymbol{L}_{\sigma}^{q^{\prime}(\omega)}} \mu_{k}(t)\right. & \\
& \left.-\left\langle f_{k},\left.\frac{\partial \psi_{i j}^{*}}{\partial \nu}\right|_{\Gamma}\right\rangle_{L^{q}(\Gamma), L^{q^{\prime}}(\Gamma)} \nu_{k}(t)\right] \boldsymbol{\Phi}_{i j} .
\end{aligned}
$$

$\mathrm{Eq}(4.23)$ is the perfect counterpart of [62, Eq (6.24)].

On the basis of expansion (4.23), we introduce the $\ell_{i} \times K$ matrix $W_{i}$ and the $\ell_{i} \times K$ matrix $U_{i}, i=1, \ldots, M$

$$
\begin{gathered}
W_{i}=\left[\begin{array}{ccc}
\left\langle f_{1},\left.\partial_{\nu} \psi_{i 1}^{*}\right|_{\Gamma}\right\rangle_{\widetilde{\Gamma}} & \cdots & \left\langle f_{K},\left.\partial_{\nu} \psi_{i 1}^{*}\right|_{\Gamma}\right\rangle_{\widetilde{\Gamma}} \\
\left\langle f_{1},\left.\partial_{\nu} \psi_{i 2}^{*}\right|_{\Gamma}\right\rangle_{\widetilde{\Gamma}} & \cdots & \left\langle f_{K},\left.\partial_{\nu} \psi_{i 2}^{*}\right|_{\Gamma}\right\rangle_{\widetilde{\Gamma}} \\
\vdots & & \vdots \\
\left\langle f_{1},\left.\partial_{\nu} \psi_{i \ell_{i}}^{*}\right|_{\Gamma}\right\rangle_{\widetilde{\Gamma}} & \cdots & \left\langle f_{K},\left.\partial_{\nu} \psi_{i \ell_{i}}^{*}\right|_{\Gamma}\right\rangle_{\widetilde{\Gamma}}
\end{array}\right]: \ell_{i} \times K\langle,\rangle_{\widetilde{\Gamma}}=\langle,\rangle_{L^{q}(\widetilde{\Gamma}), L^{\prime}(\widetilde{\Gamma})} \\
U_{i}=\left[\begin{array}{ccc}
\left\langle\boldsymbol{u}_{1}, \boldsymbol{\varphi}_{i 1}^{*}\right\rangle_{\omega} & \cdots & \left\langle\boldsymbol{u}_{K}, \boldsymbol{\varphi}_{i 1}^{*}\right\rangle_{\omega} \\
\left\langle\boldsymbol{u}_{1}, \boldsymbol{\varphi}_{i 2}^{*}\right\rangle_{\omega} & \cdots & \left\langle\boldsymbol{u}_{K}, \boldsymbol{\varphi}_{i 2}^{*}\right\rangle_{\omega} \\
\vdots & & \vdots \\
\left\langle\boldsymbol{u}_{1}, \boldsymbol{\varphi}_{i \ell_{i}}^{*}\right\rangle_{\omega} & \cdots & \left\langle\boldsymbol{u}_{K}, \boldsymbol{\varphi}_{i \ell_{i}}^{*}\right\rangle_{\omega}
\end{array}\right]: \ell_{i} \times K\langle,\rangle_{\omega}=\langle,\rangle_{\boldsymbol{L}_{\sigma}^{q}(\omega)} \\
W=\left\|\left\langle f_{r},\left.\partial_{\nu} \psi_{i j}^{*}\right|_{\Gamma}\right\rangle_{\widetilde{\Gamma}}\right\|=\left[\begin{array}{c}
W_{1} \\
W_{2} \\
\vdots \\
W_{M}
\end{array}\right] ; N \times K, \quad U=\left\|\left\langle\boldsymbol{u}_{r}, \boldsymbol{\varphi}_{i j}^{*}\right\rangle_{\omega}\right\|=\left[\begin{array}{c}
U_{1} \\
U_{2} \\
\vdots \\
U_{M}
\end{array}\right]: N \times K .
\end{gathered}
$$


Next, we represent the $N$-dimensional vector $\boldsymbol{w}_{N} \in\left(\boldsymbol{W}_{\sigma}^{q}\right)_{N}^{u}$ given by (4.12) as column vector $\hat{\boldsymbol{w}}=\left[\boldsymbol{w}_{N}\right]_{\beta}$, with respect to the basis $\beta$ in (4.13b). Rewrite (4.12) as

$$
\begin{aligned}
& \boldsymbol{w}_{N}=\sum_{i, j=1}^{M, \ell_{i}} \boldsymbol{w}_{N}^{i j} \boldsymbol{\Phi}_{i j} ; \text { set } \hat{\boldsymbol{w}}=\operatorname{col}\left[\boldsymbol{w}_{N}^{11}, \ldots, \boldsymbol{w}_{N}^{1 \ell_{1}}, \ldots, \boldsymbol{w}_{N}^{i 1}, \ldots, \boldsymbol{w}_{N}^{i \ell_{i}}, \ldots,\right. \\
&\left.\boldsymbol{w}_{N}^{M 1}, \ldots, \boldsymbol{w}_{N}^{M \ell_{M}}\right]
\end{aligned}
$$

$N=\ell_{1}+\cdots+\ell_{M}$. Thus, by (4.22)-(4.27) we can write

$$
\left[P_{N}\left[\begin{array}{c}
P_{q}(m \boldsymbol{u}) \\
\mathcal{B}_{q} D v
\end{array}\right]\right]_{\beta}=\left[\begin{array}{c}
W_{1} \\
W_{2} \\
\vdots \\
W_{M}
\end{array}\right] \hat{\nu}_{K}+\left[\begin{array}{c}
U_{1} \\
U_{2} \\
\vdots \\
U_{M}
\end{array}\right] \hat{\mu}_{k}: N \times 1, \quad \hat{\nu}_{K}=\left[\begin{array}{c}
\nu_{K}^{1} \\
\vdots \\
\nu_{K}^{k} \\
\vdots \\
\nu_{K}^{K}
\end{array}\right], \hat{\mu}_{K}=\left[\begin{array}{c}
\mu_{K}^{1} \\
\vdots \\
\mu_{K}^{k} \\
\vdots \\
\mu_{K}^{K}
\end{array}\right] .
$$

Then, in $\mathbb{C}^{N}$, with respect to the basis $\beta$ of normalized eigenvectors of $\mathbb{A}_{q, N}^{u}$, we may rewrite system (4.23) via (4.14) and (4.28) as

$$
\hat{\boldsymbol{w}}_{N}^{\prime}=\Lambda \hat{\boldsymbol{w}}_{N}+W \hat{\nu}_{K}+U \hat{\mu}_{K}=\Lambda \hat{\boldsymbol{w}}_{N}+B\left[\begin{array}{c}
\hat{\nu}_{K} \\
\hat{\mu}_{K}
\end{array}\right], \quad B=[W, U]: N \times 2 K .
$$

Then, the operator system (4.23) on $\left(\boldsymbol{W}_{\sigma}^{q}\right)_{N}^{u}$ has in (4.29) its representation in $\mathbb{C}^{N}$, characterized by the pair $\{\Lambda, B\}$, with $\Lambda$ the free dynamics matrix and $B$ the control matrix. Our next goal is to guarantee that the pair $\{\Lambda, B\}$ is controllable.

We then obtain the following counterpart of [62, Theorem 6.1].

Theorem 4.1. Assume (4.11); that is, that $\mathbb{A}_{q, N}^{u}$ is semisimple on $\left(\boldsymbol{W}_{\sigma}^{q}\right)_{N}^{u}$. System (4.29) i.e. the pair $\{\Lambda, B\}$ is controllable in case

$$
\operatorname{rank}\left[W_{i}, U_{i}\right]=\ell_{i}, i=1, \ldots, M .
$$

In fact, with reference to (4.19) and (4.20), it is possible to select boundary vectors $f_{1}, \ldots, f_{K} \in \mathcal{F} \subset$ $W^{2-{ }^{1 / q}, q}(\widetilde{\Gamma})$ with support on $\widetilde{\Gamma}$, and interior vectors $\boldsymbol{u}_{1}, \ldots, \boldsymbol{u}_{K} \in \boldsymbol{L}_{\sigma}^{q}(\omega)$ with support on $\omega$ such that (4.30) holds true, specifically

$$
\begin{aligned}
& \operatorname{rank}\left[W_{i}, U_{i}\right]= \\
& \operatorname{rank}\left[\begin{array}{ccc:ccc}
\left\langle f_{1},\left.\partial_{\nu} \psi_{i 1}^{*}\right|_{\Gamma}\right\rangle_{\widetilde{\Gamma}} & \cdots & \left\langle f_{K},\left.\partial_{\nu} \psi_{11}^{*}\right|_{\Gamma}\right\rangle_{\widetilde{\Gamma}} & \left\langle\boldsymbol{u}_{1}, \boldsymbol{\varphi}_{i 1}^{*}\right\rangle_{\omega} & \cdots & \left\langle\boldsymbol{u}_{K}, \boldsymbol{\varphi}_{i 1}^{*}\right\rangle_{\omega} \\
\left\langle f_{1},\left.\partial_{\nu} \psi_{i 2}^{*}\right|_{\Gamma}\right\rangle_{\widetilde{\Gamma}} & \cdots & \left\langle f_{K},\left.\partial_{\nu} \psi_{i 2}^{*}\right|_{\Gamma}\right\rangle_{\widetilde{\Gamma}} & \left\langle\boldsymbol{u}_{1}, \boldsymbol{\varphi}_{i 2}^{*}\right\rangle_{\omega} & \cdots & \left\langle\boldsymbol{u}_{K}, \boldsymbol{\varphi}_{i 2}^{*}\right\rangle_{\omega} \\
\vdots & & \vdots & \vdots & & \vdots \\
\left\langle f_{1},\left.\partial_{\nu} \psi_{i \ell_{i}}^{*}\right|_{\Gamma}\right\rangle_{\widetilde{\Gamma}} & \cdots & \left\langle f_{K},\left.\partial_{\nu} \psi_{i \ell_{i}}^{*}\right|_{\Gamma}\right\rangle_{\widetilde{\Gamma}} & \left\langle\boldsymbol{u}_{1}, \boldsymbol{\varphi}_{i \ell_{i}}^{*}\right\rangle_{\omega} & \cdots & \left\langle\boldsymbol{u}_{K}, \boldsymbol{\varphi}_{i \ell_{i}}^{*}\right\rangle_{\omega}
\end{array}\right]
\end{aligned}
$$

Proof. This is the counterpart of the proof [61, Theorem 6.1]. As in that paper, the present proof rests on a suitable Unique Continuation Property, Theorem B.1 of Appendix B as documented below.

In general. in seeking that the $\ell_{i}$ rows (of length $2 K$ ) in matrix (4.31) be linearly independent, we see that the full rank statement (4.31) will hold true if and only if we can exclude that each of the two sets of vectors

$$
\left\{\partial_{\nu} \psi_{i 1}^{*}, \ldots, \partial_{\nu} \psi_{i \ell_{i}}^{*}\right\} \text { in } L^{q}(\widetilde{\Gamma}) \text { and }\left\{\boldsymbol{\varphi}_{i 1}^{*}, \ldots, \boldsymbol{\varphi}_{i \ell_{i}}^{*}\right\} \text { in } \boldsymbol{L}_{\sigma}^{q}(\omega)
$$


are linearly independent with the same linear independence relation in the two cases; that is, if and only if we can establish that we cannot have simultaneously

$$
\partial_{\nu} \psi_{i \ell_{i}}^{*}=\sum_{j=1}^{\ell_{i}-1} \alpha_{j} \partial_{\nu} \psi_{i j}^{*} \text { in } L^{q}(\widetilde{\Gamma}) \quad \text { and } \quad \boldsymbol{\varphi}_{i \ell_{i}}^{*}=\sum_{j=1}^{\ell_{i}-1} \alpha_{j} \boldsymbol{\varphi}_{i j}^{*} \text { in } \boldsymbol{L}_{\sigma}^{q}(\omega)
$$

with the same constants $\alpha_{1}, \ldots, \alpha_{\ell_{i}-1}$ in both expansions [61].

Claim: Statement (4.33) is false. By contradiction, suppose that both linear combinations in (4.33) hold true. Define the $(d+1)$-vector $\boldsymbol{\Phi}^{*}=\left\{\boldsymbol{\varphi}^{*}, \psi^{*}\right\}$ (depending on $i$ ) on $\boldsymbol{L}_{\sigma}^{q}(\Omega) \times L^{q}(\Omega)$ by setting

$$
\boldsymbol{\Phi}^{*} \equiv\left[\begin{array}{c}
\boldsymbol{\varphi}^{*} \\
\psi^{*}
\end{array}\right] \equiv \sum_{j=1}^{\ell_{i}-1}\left[\begin{array}{c}
\alpha_{j} \boldsymbol{\varphi}_{i j}^{*} \\
\alpha_{j} \psi_{i j}^{*}
\end{array}\right]-\left[\begin{array}{c}
\boldsymbol{\varphi}_{i \ell_{i}}^{*} \\
\psi_{i \ell_{i}}^{*}
\end{array}\right]=\sum_{j=1}^{\ell_{i}-1} \alpha_{j} \boldsymbol{\Phi}_{i j}^{*}-\boldsymbol{\Phi}_{i \ell_{i}}^{*}, i=1, \ldots, M ; q \geq 2 .
$$

Thus, in view of (4.33), we obtain

$$
\varphi^{*} \equiv 0 \text { in } \omega,\left.\quad \partial_{\nu} \psi^{*}\right|_{\widetilde{\Gamma}} \equiv 0 .
$$

Moreover, since $\boldsymbol{\Phi}_{i j}^{*}=\left\{\boldsymbol{\varphi}_{i j}^{*}, \psi_{i j}^{*}\right\}$ is an eigenvector of the operator $\mathbb{A}_{q, N}^{*}$ corresponding to the (unstable) eigenvalue $\bar{\lambda}_{i}$, then so is $\boldsymbol{\Phi}^{*}: \mathbb{A}_{q, N}^{*} \boldsymbol{\Phi}^{*}=\bar{\lambda}_{i} \boldsymbol{\Phi}^{*}$. Then $\boldsymbol{\Phi}^{*}=\left\{\boldsymbol{\varphi}^{*}, \psi^{*}\right\}$ satisfies the corresponding PDE version of the eigenvector identity as given by (4.10), see (B.4) in Appendix B

$$
\left\{\begin{aligned}
-\nu \Delta \boldsymbol{\varphi}^{*}+L_{e}^{*}\left(\boldsymbol{\varphi}^{*}\right)+\psi^{*} \nabla \theta_{e}+\nabla \pi & =\bar{\lambda}_{i} \boldsymbol{\varphi}^{*} & & \text { in } \Omega \\
-\kappa \Delta \psi^{*}-\boldsymbol{y}_{e} \cdot \nabla \psi^{*}-\gamma \boldsymbol{\varphi}^{*} \cdot \boldsymbol{e}_{d} & =\bar{\lambda}_{i} \psi^{*} & & \text { in } \Omega \\
\operatorname{div} \boldsymbol{\varphi}^{*} & =0 & & \text { in } \Omega \\
\left.\boldsymbol{\varphi}^{*}\right|_{\Gamma}=0,\left.\psi^{*}\right|_{\Gamma} & =0 & & \text { on } \Gamma .
\end{aligned}\right.
$$

and in addition, the over-determined conditions in (4.35):

$$
\varphi^{*} \equiv 0 \text { in } \omega,\left.\quad \partial_{\nu} \psi^{*}\right|_{\widetilde{\Gamma}} \equiv 0 .
$$

Write $\boldsymbol{\varphi}^{*}=\left\{\varphi^{*(1)}, \varphi^{*(2)}, \ldots, \varphi^{*(d)}\right\}$ for the $d$-components of the vector $\boldsymbol{\varphi}^{*}$. Since $\boldsymbol{e}_{d}=\{0, \ldots, 0,1\}$, the property $\varphi^{* d}=0$ in $\omega$, contained in (4.36e), used in (4.36b) implies:

$$
\left\{\begin{array}{lr}
\kappa \Delta \psi^{*}+\boldsymbol{y}_{e} \cdot \nabla \psi^{*}=-\bar{\lambda}_{i} \psi^{*} & \text { in } \omega \\
\left.\psi^{*}\right|_{\widetilde{\Gamma}}=0,\left.\partial_{\nu} \psi^{*}\right|_{\widetilde{\Gamma}}=0 & \text { on } \widetilde{\Gamma} .
\end{array}\right.
$$

recalling the boundary conditions for $\psi^{*}$ in (4.36d) and (4.36e) for the $\psi^{*}$-problem defined on $\omega$, with $\widetilde{\Gamma} \subset \partial \omega$, as in Fig 1. It is then a standard result [17, [?, Sect 19, pp 59-61], [36, p 3], 51] that the over-determined problem (4.37) implies

$$
\psi^{*} \equiv 0 \text { in } \omega .
$$

Then (4.38) and the full strength of (4.36d) give the over-determination

$$
\varphi^{*} \equiv 0 \text { in } \omega, \psi^{*} \equiv 0 \text { in } \omega
$$

for problem (4.36). By means of such over-determination, we can apply the Unique Continuation Property [89, Theorem 5] recalled as Theorem B.1 in Appendix B and conclude that

$$
\boldsymbol{\Phi}^{*}=\left\{\boldsymbol{\varphi}^{*}, \psi^{*}\right\} \equiv 0 \text { in } \Omega, \quad \pi \equiv \text { const. in } \Omega,
$$


or recalling (4.34)

$$
\boldsymbol{\Phi}_{i \ell_{i}}^{*}=\sum_{j=1}^{\ell_{i}-1} \alpha_{j} \boldsymbol{\Phi}_{i j}^{*} \text { in } \boldsymbol{W}_{\sigma}^{q}(\Omega)=\boldsymbol{L}_{\sigma}^{q}(\Omega) \times L^{q}(\Omega) ;
$$

i.e. the set $\left\{\boldsymbol{\Phi}_{i 1}^{*}, \ldots, \boldsymbol{\Phi}_{i \ell_{i}}^{*}\right\}$ is linearly dependent on $\boldsymbol{W}_{\sigma}^{q}(\Omega)$. But this is false by the very selection of such eigenvectors, see above (4.1). Thus the two conditions in (4.33) cannot hold simultaneously. The claim is proved. Hence it is possible to select, in infinitely many ways, boundary functions $f_{1}, \ldots, f_{K} \in$ $\mathcal{F} \subset L^{q}(\Gamma)$ for $q \geq 2$ and interior $d$-vectors $\boldsymbol{u}_{1}, \ldots, \boldsymbol{u}_{K}$ in $\boldsymbol{L}_{\sigma}^{q}(\Omega)$, such that the Kalman algebraic full rank conditions (4.31) hold true. Indeed they may be chosen independent of $i$. Start with (4.31) for $\bar{\imath}$ such that $\ell_{\bar{\imath}}=K=\max \left\{\ell_{i}, i=1, \ldots, M\right\}$ yielding $f_{1}, \ldots, f_{K}$ which are linearly independent. They also work in other $i$ 's.

Remark 4.1. The UCP in [89, Theorem 5] reported as Theorem B.1] in Appendix B refers to problem (4.36a-C), without the B.C. (4.36d), with the a-priori over-determination $\psi^{*} \equiv 0$ in $\omega$, and $\left\{\varphi^{*(1)}, \ldots, \varphi^{*(d-1)}\right\} \equiv$ 0 in $\omega$, thus not involving the last $d$-component $\varphi^{*(d)} \equiv 0$. In contrast, in the setting of proving the above Claim, we need $\varphi^{*(d)} \equiv 0$ in $\omega$ in order to deduce $\psi^{*} \equiv 0$ in $\omega$. The present proof on the validity of the rank conditions (4.31), ultimately relying on the UCP of [89, Theorem 5] in the Appendix B requires the full strength of the fluid vectors $\boldsymbol{u}_{1}, \ldots, \boldsymbol{u}_{\ell_{i}}$, each possessing $d$-components. No geometrical assumptions are involved for the pair $\{\omega, \widetilde{\Gamma}\}$, except for $\omega$ being an interior subdomain touching the boundary $\widetilde{\Gamma}$ as in Fig 1 . In Appendix $[\mathbf{C}$, we report an improvement requiring only $(d-1)$ components from the fluid vectors $\boldsymbol{u}_{1}, \ldots, \boldsymbol{u}_{\ell_{i}}$ to include necessarily the $d^{\text {th }}$ components.

Remark 4.2. We have established Theorem 4.1 under the simplifying semisimple assumption (4.11). However, Theorem 4.1 holds true in full generality. The corresponding proof is lengthy and technical. It may be given by following the scheme given in [56], [57, 62 for the Navier-Stokes equations. It requires use of the controllability criterion for a finite dimensional pair $\{A, B\}$ with $A$ in Jordan canonical form [18, 8. Henceforth, we proceed by taking that Theorem 4.1 is true in full generality.

As a consequence Theorem 4.1 we obtain the following fundamental result on the uniform stabilization with arbitrarily preassigned decay rate, of the original unstable, finite dimensional $\boldsymbol{w}_{N}$-problem (3.6a), the counterpart of [61, Theorem 5.1].

Theorem 4.2. Let $\lambda_{1}, \ldots, \lambda_{M}$ be the unstable distinct eigenvalues of the operator $\mathbb{A}_{q}\left(=\mathbb{A}_{q, N}^{u}\right)$ as in (1.34) with geometric multiplicity $\ell_{i}, i=1, \ldots, M$, and set $K=\sup \left\{\ell_{i} ; i=1, \ldots, M\right\}$. Let $\widetilde{\Gamma}$ be an open connected subset of the boundary $\Gamma$ of positive surface measure and $\omega$ be a localized collar supported by $\widetilde{\Gamma}$ (Fig. 1). Let $q \geq 2$. Given $\gamma_{1}>0$ arbitrarily large, we can construct two $K$-dimensional controllers: a boundary control $v=v_{N}$ acting with support on $\widetilde{\Gamma}$, of the form given by

$$
v=v_{N}=\sum_{k=1}^{K} \nu_{k}(t) f_{k}, f_{k} \in \mathcal{F} \subset W^{2-1 / q, q}(\Gamma), q \geq 2,
$$

$\mathcal{F}$ defined in (4.21), $q \geq 2, f_{k}$ supported on $\widetilde{\Gamma}$, and an interior control $\boldsymbol{u}=\boldsymbol{u}_{N}$ acting on $\omega$, of the form given by

$$
\boldsymbol{u}=\boldsymbol{u}_{N}=\sum_{k=1}^{K} \mu_{k}(t) \boldsymbol{u}_{k}, \quad \boldsymbol{u}_{k} \in\left(\boldsymbol{W}_{\sigma}^{q}\right)_{N}^{u} \subset \boldsymbol{L}_{\sigma}^{q}(\Omega), \quad \mu_{k}(t)=\text { scalar },
$$

thus with interior vectors $\left[\boldsymbol{u}_{1}, \ldots, \boldsymbol{u}_{K}\right]$ in the smooth subspace $\left(\boldsymbol{W}_{\sigma}^{q}\right)_{N}^{u}$ of $\boldsymbol{L}_{\sigma}^{q}(\Omega), 2 \leq q<\infty$, supported on $\omega$, such that, once inserted in the finite dimensional projected $\boldsymbol{w}_{N}$-system in (3.6a), yields the system

$$
\boldsymbol{w}_{N}^{\prime}=\mathbb{A}_{q, N}^{u} \boldsymbol{w}_{N}+P_{N}\left[\begin{array}{c}
P_{q}\left(m\left(\sum_{k=1}^{K} \mu_{k}(t) \boldsymbol{u}_{k}\right)\right) \\
\mathcal{B}_{q} D\left(\sum_{k=1}^{K} \nu_{k}(t) f_{k}\right)
\end{array}\right],
$$


whose solution then satisfies the estimate

$$
\begin{aligned}
& \left\|\boldsymbol{w}_{N}(t)\right\|_{\boldsymbol{L}_{\sigma}^{q}(\Omega)}+\left\|v_{N}(t)\right\|_{L^{q}(\widetilde{\Gamma})}+\left\|v_{N}^{\prime}(t)\right\|_{L^{q}(\widetilde{\Gamma})}+ \\
& \quad\left\|\boldsymbol{u}_{N}(t)\right\|_{\boldsymbol{L}_{\sigma}^{q}(\omega)}+\left\|\boldsymbol{u}_{N}^{\prime}(t)\right\|_{\boldsymbol{L}_{\sigma}^{q}(\omega)} \leq C_{\gamma_{1}} e^{-\gamma_{1} t}\left\|P_{N} w_{0}\right\|_{\boldsymbol{W}_{\sigma}^{q}(\Omega)}, \quad t \geq 0 .
\end{aligned}
$$

Moreover, such controllers $v=v_{N}$ and $\boldsymbol{u}=\boldsymbol{u}_{N}$ may be chosen in feedback form: that is, with reference to the explicit expressions (4.42) for $v$ and (4.43) for $\boldsymbol{u}$, of the form $\nu_{k}(t)=\left\langle\boldsymbol{w}_{N}(t), \boldsymbol{p}_{k}\right\rangle_{\left(\boldsymbol{W}_{\sigma}^{q}\right)_{N}^{u}}$ and $\mu_{k}(t)=\left\langle\boldsymbol{w}_{N}(t), \boldsymbol{q}_{k}\right\rangle_{\left(\boldsymbol{W}_{\sigma}^{q}\right)_{N}^{u}}$ for suitable vectors $\boldsymbol{p}_{k} \in\left(\left(\boldsymbol{W}_{\sigma}^{q}\right)_{N}^{u}\right)^{*} \subset \boldsymbol{L}_{\sigma}^{q^{\prime}}(\Omega) \times L^{q^{\prime}}(\Omega), \boldsymbol{q}_{k} \in\left(\left(\boldsymbol{W}_{\sigma}^{q}\right)_{N}^{u}\right)^{*} \subset$ $\boldsymbol{L}_{\sigma}^{q^{\prime}}(\Omega) \times L^{q^{\prime}}(\Omega)$ depending on $\gamma_{1}$, where $\langle$,$\rangle denotes the duality pairing \left(\boldsymbol{W}_{\sigma}^{q}\right)_{N}^{u} \times\left(\left(\boldsymbol{W}_{\sigma}^{q}\right)_{N}^{u}\right)^{*}$.

In conclusion, $\boldsymbol{w}_{N}$ in (4.45) is the solution of the equation (4.44) on $\left(\boldsymbol{W}_{\sigma}^{q}\right)_{N}^{u}$ rewritten explicitly as

$$
\boldsymbol{w}_{N}^{\prime}=\mathbb{A}_{q, N}^{u} \boldsymbol{w}_{N}+P_{N}\left[\begin{array}{c}
P_{q}\left(m\left(\sum_{k=1}^{K}\left\langle\boldsymbol{w}_{N}(t), \boldsymbol{q}_{k}\right\rangle_{\left(\boldsymbol{W}_{\sigma}^{q}\right)_{N}^{u}} \boldsymbol{u}_{k}\right)\right) \\
\mathcal{B}_{q} D\left(\sum_{k=1}^{K}\left\langle\boldsymbol{w}_{N}(t), \boldsymbol{p}_{k}\right\rangle_{\left(\boldsymbol{W}_{\sigma}^{q}\right)_{N}^{u}} f_{k}\right)
\end{array}\right],
$$

$f_{k}$ supported on $\widetilde{\Gamma}, \boldsymbol{u}_{k}$ supported on $\omega$, rewritten in turn as

$$
\boldsymbol{w}_{N}^{\prime}=\bar{A}^{u} \boldsymbol{w}_{N}, \boldsymbol{w}_{N}(t)=e^{\bar{A}^{u} t} P_{N} \boldsymbol{w}_{0}, \boldsymbol{w}_{N}(0)=P_{N} \boldsymbol{w}_{0} \quad \text { on }\left(\boldsymbol{W}_{\sigma}^{q}\right)_{N}^{u} .
$$

Proof. The technical proof is similar circumstances was given in [56], [57, 62]. Thus, we give here only some insight. The key new fact is the controllability test of Theorem 4.1 Having established the controllability condition for the pair $\{\Lambda, B\}$, then by the well-known Popov's criterion on finite dimensional theory (see e.g. [94, Theorem $2.9 \mathrm{p} \mathrm{44]}$ ), there exists a feedback matrix $Q: 2 K \times N$, such that the spectrum of the matrix $[\Lambda+B Q]$ may be arbitrarily preassigned, in particular, to lie arbitrarily on the left half-plane $\left\{\lambda: \operatorname{Re} \lambda<-\gamma_{1}<-\operatorname{Re} \lambda_{N+1}\right\}$, with $\gamma_{1}>0$ preassigned, as desired. The resulting closed-loop system is

$$
\begin{gathered}
\left(\hat{\boldsymbol{w}}_{N}\right)^{\prime}=\Lambda \hat{\boldsymbol{w}}_{N}+B Q \hat{\boldsymbol{w}}_{N},\left[\begin{array}{c}
\hat{\nu}_{K} \\
\hat{\mu}_{K}
\end{array}\right]=Q \hat{\boldsymbol{w}}_{N}=\text { feedback control } \\
Q=\left[\begin{array}{c}
\operatorname{row} \hat{p}_{1} \\
\vdots \\
\operatorname{row} \hat{p}_{K} \\
\operatorname{row} \hat{q}_{1} \\
\vdots \\
\operatorname{row} \hat{q}_{K}
\end{array}\right]: 2 K \times N ; \hat{\nu}_{K}=\left[\begin{array}{c}
\nu_{K}^{1}=\left\langle\hat{\boldsymbol{p}}_{1}, \hat{\boldsymbol{w}}_{N}\right\rangle \\
\vdots \\
\nu_{K}^{k}=\left\langle\hat{\boldsymbol{p}}_{k}, \hat{\boldsymbol{w}}_{N}\right\rangle \\
\vdots \\
\nu_{K}^{K}=\left\langle\hat{\boldsymbol{p}}_{K}, \hat{\boldsymbol{w}}_{N}\right\rangle
\end{array}\right], \hat{\mu}_{K}=\left[\begin{array}{c}
\mu_{K}^{1}=\left\langle\hat{\boldsymbol{q}}_{1}, \hat{\boldsymbol{w}}_{N}\right\rangle \\
\vdots \\
\mu_{K}^{k}=\left\langle\hat{\boldsymbol{q}}_{k}, \hat{\boldsymbol{w}}_{N}\right\rangle \\
\vdots \\
\mu_{K}^{K}=\left\langle\hat{\boldsymbol{q}}_{K}, \hat{\boldsymbol{w}}_{N}\right\rangle
\end{array}\right]
\end{gathered}
$$

in the $\mathbb{C}^{N}$-inner product. Thus, returning from $\mathbb{C}^{N} \times \mathbb{C}^{N}$ back to $\left(\boldsymbol{W}_{\sigma}^{q}\right)_{N}^{u} \times\left[\left(\boldsymbol{W}_{\sigma}^{q}\right)_{N}^{u}\right]^{*}$, there exist vectors $\boldsymbol{p}_{1}, \ldots, \boldsymbol{p}_{K}$ and $\boldsymbol{q}_{1}, \ldots, \boldsymbol{q}_{K}$ in $\left[\left(\boldsymbol{W}_{\sigma}^{q}\right)_{N}^{u}\right]^{*}$ such that

$$
\nu_{K}^{k}=\left\langle\boldsymbol{w}_{N}, \boldsymbol{p}_{k}\right\rangle, \mu_{K}^{k}=\left\langle\boldsymbol{w}_{N}, \boldsymbol{q}_{k}\right\rangle, k=1, \ldots, K
$$

where $\langle$,$\rangle denotes the duality paring \left(\boldsymbol{W}_{\sigma}^{q}\right)_{N}^{u} \times\left[\left(\boldsymbol{W}_{\sigma}^{q}\right)_{N}^{u}\right]^{*}$. This way, the closed loop system (4.46) corresponds to the $\mathbb{C}^{N}$-system (4.48). (4.49). 


\section{The linearized $w$-system $(1.32)$ in feedback form.}

We return to the open-loop linearized $\boldsymbol{w}$-problem (1.31), (1.32b), and use the same corresponding feedback operators

$$
\begin{gathered}
v=F \boldsymbol{w}=\sum_{k=1}^{K}\left\langle P_{N} \boldsymbol{w}, \boldsymbol{p}_{k}\right\rangle f_{k}, f_{k} \in \mathcal{F} \subset W^{2-{ }^{1 / q}, q}(\Gamma), \\
\boldsymbol{p}_{k} \in\left(\boldsymbol{W}_{N}^{u}\right)^{*} \subset \boldsymbol{L}_{\sigma}^{q^{\prime}}(\Omega) \times L^{q}(\Omega), q \geq 2, f_{k} \text { supported on } \widetilde{\Gamma} . \\
J \boldsymbol{w}=P_{q} m(\boldsymbol{u})=P_{q} m\left(\sum_{k=1}^{K}\left\langle P_{N} \boldsymbol{w}, \boldsymbol{q}_{k}\right\rangle \boldsymbol{u}_{k}\right), \\
\boldsymbol{q}_{k} \in\left(\boldsymbol{W}_{N}^{u}\right)^{*} \subset \boldsymbol{L}_{\sigma}^{q^{\prime}}(\Omega) \times L^{q}(\Omega), \boldsymbol{u}_{k} \text { supported on } \omega,
\end{gathered}
$$

that were employed in (4.46) to uniformly stabilizing the finite dimensional $\boldsymbol{w}_{N}$-problem (3.6a) with arbitrarily preassigned decay $\gamma_{1}$ in (4.45). See also (2.2b), (2.3). We thus obtain from (1.32b) the resulting closed-loop linearized $\boldsymbol{w}$-problem in feedback form

$$
\frac{d \boldsymbol{w}}{d t}=\left[\begin{array}{cc}
\mathcal{A}_{q} & -\mathcal{C}_{\gamma} \\
-\mathcal{C}_{\theta_{e}} & 0
\end{array}\right] \boldsymbol{w}+\left[\begin{array}{c}
P_{q}\left(m \sum_{k=1}^{K}\left\langle P_{N} \boldsymbol{w}, \boldsymbol{q}_{k}\right\rangle \boldsymbol{u}_{k}\right) \\
-\mathcal{B}_{q}\left(w_{2}-D \sum_{k=1}^{K}\left\langle P_{N} \boldsymbol{w}, \boldsymbol{p}_{k}\right\rangle f_{k}\right)
\end{array}\right]
$$

(compare with (4.46) ) or by (5.1), (5.2)

$$
\frac{d \boldsymbol{w}}{d t}=\left[\begin{array}{cc}
\mathcal{A}_{q} & -\mathcal{C}_{\gamma} \\
-\mathcal{C}_{\theta_{e}} & 0
\end{array}\right] \boldsymbol{w}+\left[\begin{array}{c}
J \boldsymbol{w} \\
-\mathcal{B}_{q}\left(w_{2}-D F \boldsymbol{w}\right)
\end{array}\right] \equiv \mathbb{A}_{F, q} \boldsymbol{w}
$$

as in (2.4b). Thus $\mathbb{A}_{F, q}$ defines the linearized $\boldsymbol{w}$-problem in feedback form, while $\mathbb{A}_{q}$ in (1.34) is the free dynamics operator. We rewrite (5.3) as

$$
\frac{d \boldsymbol{w}}{d t}=\mathbb{A}_{F, q} \boldsymbol{w}=\hat{\mathbb{A}}_{F, q} \boldsymbol{w}+\Pi \boldsymbol{w}
$$

where $\widehat{\mathbb{A}}_{F, q}$ is the streamlined operator that removes benign terms from $\mathbb{A}_{F, q}$ and moves them into the perturbation operator $\Pi$

$$
\begin{gathered}
\hat{\mathbb{A}}_{F, q} \boldsymbol{w}=\left[\begin{array}{c}
-A_{q} \boldsymbol{w}_{1} \\
-\mathcal{B}_{q}\left(w_{2}-D F \boldsymbol{w}\right)
\end{array}\right], \quad \Pi \boldsymbol{w}=\left[\begin{array}{cc}
A_{o, q} & -\mathcal{C}_{\gamma} \\
-\mathcal{C}_{\theta_{e}} & 0
\end{array}\right] \boldsymbol{w}+\left[\begin{array}{c}
J \boldsymbol{w} \\
0
\end{array}\right] \\
\mathcal{D}\left(\mathbb{A}_{F, q}\right)=\mathcal{D}\left(\hat{\mathbb{A}}_{F, q}\right)=\left\{\boldsymbol{w}=\left[\begin{array}{l}
\boldsymbol{w}_{1} \\
w_{2}
\end{array}\right] \in \boldsymbol{W}_{\sigma}^{q}(\Omega)=\boldsymbol{L}_{\sigma}^{q}(\Omega) \times L^{q}(\Omega): \boldsymbol{w}_{1} \in \mathcal{D}\left(A_{q}\right),\right. \\
\left.\left(w_{2}-D F \boldsymbol{w}\right) \in \mathcal{D}\left(\mathcal{B}_{q}\right)\right\} \subset \boldsymbol{W}^{2, q}(\Omega) \cap \boldsymbol{W}_{0}^{1, q}(\Omega) \cap \boldsymbol{L}_{\sigma}^{q}(\Omega) \times W^{2, q}(\Omega) \\
\mathcal{D}\left(\mathcal{B}_{q}\right)=\mathcal{D}\left(B_{q}\right)=W^{2, q}(\Omega) \cap W_{0}^{1, q}(\Omega) ; \mathcal{D}(\Pi)=\mathcal{D}\left(A_{o, q}\right) \times L^{q}(\Omega) ; \\
D v=D F \boldsymbol{w} \in W^{2, q}(\Omega)
\end{gathered}
$$

recalling (1.14), (1.15), (1.18), (1.23c), (2.2b). 


\section{The linearized feedback operator $\mathbb{A}_{F, q}$ is the generator of a s.c. analytic, uniformly stable semigroup $e^{\mathbb{A}_{F, q}^{q} t}$ on $\boldsymbol{W}_{\sigma}^{q}(\Omega)$ and $\boldsymbol{V}_{b}^{q, p}(\Omega)$.}

Theorem 6.1. The operator $\mathbb{A}_{F, q}$ in (5.3b) generates a s.c., analytic semigroup $e^{\mathbb{A}_{F, q}{ }^{t}}$ on $\boldsymbol{W}_{\sigma}^{q}(\Omega) \equiv$ $\boldsymbol{L}_{\sigma}^{q}(\Omega) \times L^{q}(\Omega)$ as well as in $\boldsymbol{V}_{b}^{q, p}(\Omega) \equiv \widetilde{\boldsymbol{B}}_{q, p}^{2-{ }^{2} / p}(\Omega) \times B_{q, p}^{2-2 / p}(\Omega)$.

Proof. First on $\boldsymbol{W}_{\sigma}^{q}(\Omega)$ : As the perturbation operator $\Pi$ in (5.5a) involves bounded operators $\mathcal{C}_{\gamma}, \mathcal{C}_{\theta_{e}}, J$ (see (1.21), (1.22), (5.1)), as well as the benign operator $A_{o, q}$, in (1.15) with $A_{o, q} A_{q}^{-1 / 2}$ bounded on $\boldsymbol{L}_{\sigma}^{q}(\Omega)$, it suffices to show that the streamlined operator $\widehat{\mathbb{A}}_{F, q}$ in (5.5a) generates a s.c. analytic semigroup $e^{\mathbb{A}_{F, q} t}$ on $\boldsymbol{W}_{\sigma}^{q}(\Omega)$. To this end, it will be more convenient to show equivalently that the adjoint $\hat{\mathbb{A}}_{F, q}^{*}$ is the generator of a s.c. analytic semigroup $e^{\hat{\mathbb{A}}_{F, q}^{*} t}$ on $\left[\boldsymbol{W}_{\sigma}^{q}(\Omega)\right]^{*}=\boldsymbol{W}_{\sigma}^{q^{\prime}}(\Omega)=\boldsymbol{L}_{\sigma}^{q^{\prime}}(\Omega) \times L^{q^{\prime}}(\Omega)$, since $\boldsymbol{W}_{\sigma}^{q}(\Omega), 1<q<\infty$, is a reflexive space. The adjoint $\widehat{\mathbb{A}}_{F, q}^{*}$ of $\widehat{\mathbb{A}}_{F, q}$ in (5.5a) is given by

$$
\left\{\begin{array}{l}
\hat{\mathbb{A}}_{F, q}^{*}\left[\begin{array}{l}
\boldsymbol{v}_{1} \\
v_{2}
\end{array}\right]=\left[\begin{array}{cc}
-A_{q}^{*} & 0 \\
0 & -\mathcal{B}_{q}^{*}
\end{array}\right]\left[\begin{array}{l}
\boldsymbol{v}_{1} \\
v_{2}
\end{array}\right]+F^{*} D^{*} \mathcal{B}_{q}^{*} v_{2} \\
\mathcal{D}\left(\hat{\mathbb{A}}_{F, q}^{*}\right)=\mathcal{D}\left(A_{q}^{*}\right) \times \mathcal{D}\left(B_{q}^{*}\right), \mathcal{D}\left(B_{q}^{*}\right)=\mathcal{D}\left(\mathcal{B}_{q}^{*}\right)
\end{array}\right.
$$

by (1.23c). Since the operator

$$
\hat{\mathbb{A}}_{q}^{*}=\left[\begin{array}{cc}
-A_{q}^{*} & 0 \\
0 & -\mathcal{B}_{q}^{*}
\end{array}\right], \boldsymbol{W}_{\sigma}^{q^{\prime}}(\Omega) \supset \mathcal{D}\left(\hat{\mathbb{A}}_{q}^{*}\right)=\mathcal{D}\left(A_{q}^{*}\right) \times \mathcal{D}\left(\mathcal{B}_{q}^{*}\right) \longrightarrow \boldsymbol{W}_{\sigma}^{q^{\prime}}(\Omega)
$$

is plainly the generator of a s.c. analytic semigroup $e^{\hat{\mathbb{A}}_{F, q}^{*} t}$ on $\boldsymbol{W}_{\sigma}^{q^{\prime}}(\Omega)$ (Appendix $\underline{\mathrm{A}}$ ), it will suffice to show that the perturbation operator

$$
F^{*} D^{*} \mathcal{B}_{q}^{*} \text { is }\left(\hat{\mathbb{A}}_{q}^{*}\right)^{\theta_{0}}=\left[\begin{array}{cc}
-A_{q}^{* \theta_{0}} & 0 \\
0 & -\mathcal{B}_{q}^{* \theta_{0}}
\end{array}\right] \text { - bounded, for some constant } 0<\theta_{0}<1
$$

68. In our case, it will be $\theta_{0}=1-1 / 2 q+\varepsilon<1$. In fact, recall via 1.23b that $D^{*} \mathcal{B}_{q}^{* \gamma} \in$ $\mathcal{L}\left(L^{q^{\prime}}(\Omega), L^{q^{\prime}}(\Gamma)\right), \gamma=1 / 2 q-\epsilon . \quad\left(\right.$ In $[6.3)$ and below we are taking that the fractional powers of $\mathcal{B}_{q}^{*}$ are well-defined, for otherwise a translation will do it. Alternatively, in (6.1a), write $\mathcal{B}_{q}^{*}=B_{q}^{*}+B_{o, q}^{*}$ from (1.18) as in (B.10) for $\kappa=1$ where $\mathcal{D}\left(B_{o, q}^{*}\right)=\mathcal{D}\left(B_{q}^{*^{1} / 2}\right)$. Do the argument with $B_{q}^{*}$ whose fractional powers are well-defined. This introduces an additional perturbation which is benign, as it can be handled by using

$$
\left\|B_{o, q}^{*} v_{2}\right\|=\left\|\left(B_{o, q}^{*} B_{q}^{*-1 / 2}\right)\left(B_{q}^{*-1 / 2} B_{q}^{*}\right) v_{2}\right\| \leq C\left\|B_{q}^{*} v_{2}\right\|
$$

as desired.) Next, for $v_{2} \in \mathcal{D}\left(\mathcal{B}_{q}^{* 1-\gamma}\right)$ and $\boldsymbol{v}_{1} \in \mathcal{D}\left(A_{q}^{* 1-\gamma}\right)$, we estimate since $F^{*} \in \mathcal{L}\left(L^{q^{\prime}}(\Gamma), \boldsymbol{W}_{\sigma}^{q^{\prime}}(\Omega)\right)$ in the norm of $\boldsymbol{W}_{\sigma}^{q^{\prime}}(\Omega)$ :

$$
\begin{aligned}
\left\|F^{*} D^{*} \mathcal{B}_{q}^{*} v_{2}\right\| & =\left\|\left(F^{*} D^{*} \mathcal{B}_{q}^{*^{\gamma}}\right) \mathcal{B}_{q}^{*^{1-\gamma}} v_{2}\right\| \leq C\left\|\mathcal{B}_{q}^{*^{1-\gamma}} v_{2}\right\| \\
& \leq C\left[\left\|\mathcal{B}_{q}^{*^{1-\gamma}} v_{2}\right\|+\left\|A_{q}^{*^{1-\gamma}} \boldsymbol{v}_{1}\right\|\right] \\
& \leq C\left\|\left[\begin{array}{cc}
A_{q}^{*} & 0 \\
0 & B_{q}^{*}
\end{array}\right]^{1-\gamma}\left[\begin{array}{l}
\boldsymbol{v}_{1} \\
v_{2}
\end{array}\right]\right\|
\end{aligned}
$$


and (6.6) proves (6.3). In conclusion: the operator $\mathbb{A}_{F, q}^{*}$ generates a s.c. analytic semigroup on $\left(\boldsymbol{W}_{\sigma}^{q}(\Omega)^{*}\right)=\boldsymbol{L}_{\sigma}^{q^{\prime}}(\Omega) \times L^{q^{\prime}}(\Omega)$; and hence as this space is reflexive, the operator $\mathbb{A}_{F, q}$ generates a s.c. analytic semigroup on $\boldsymbol{W}_{\sigma}^{q}(\Omega)=\boldsymbol{L}_{\sigma}^{q}(\Omega) \times L^{q}(\Omega)$.

$\underline{\text { Next on } \boldsymbol{V}_{b}^{q, p}(\Omega) \equiv \widetilde{\boldsymbol{B}}_{q, p}^{2-{ }^{2} / p}(\Omega) \times B_{q, p}^{2-2 / p}(\Omega)}$ : (See Remark 1.3) From the above conclusion on $\mathbb{A}_{F, q}^{*}$ on $\left(\boldsymbol{W}_{\sigma}^{q}(\Omega)\right)^{*}$, it then follows that $\mathbb{A}_{F, q}^{*}$ generates a s.c. analytic semigroup on $\mathcal{D}\left(\mathbb{A}_{F, q}^{*}\right) \equiv \mathcal{D}\left(A_{q}^{*}\right) \times \mathcal{D}\left(B_{q}^{*}\right)$, see (6.1b). Then $\mathbb{A}_{F, q}^{*}$ generates a s.c. analytic semigroup on the real interpolation spaces between $\mathcal{D}\left(A_{q}^{*}\right) \times \mathcal{D}\left(B_{q}^{*}\right)$ and $\boldsymbol{L}_{\sigma}^{q^{\prime}}(\Omega) \times L^{q^{\prime}}(\Omega)$, thus on the space $\widetilde{\boldsymbol{B}}_{q^{\prime}, p}^{2-{ }^{2} / p}(\Omega) \times B_{q^{\prime}, p}^{2-^{2} / p}(\Omega), 1<p<{ }^{2 q^{\prime}} / 2 q^{\prime}-1$. Recall the version of (1.11) for adjoints and get

$$
\left(\boldsymbol{L}_{\sigma}^{q^{\prime}}(\Omega), \mathcal{D}\left(A_{q}^{*}\right)\right)_{1-\frac{1}{p}, p} \equiv \widetilde{\boldsymbol{B}}_{q^{\prime}, p}^{2-^{2} / p}(\Omega), 1<p<\frac{2 q^{\prime}}{2 q^{\prime}-1},
$$

$$
1<q^{\prime}<2, q>2, \frac{1}{q}+\frac{1}{q^{\prime}}=1 .
$$

For the second component recall Remark 1.3. It then finally follows, again by reflexivity of the space, that $\mathbb{A}_{F, q}$ generates a s.c. analytic semigroup on the space $\boldsymbol{V}_{b}^{q, p}(\Omega) \equiv \widetilde{\boldsymbol{B}}_{q, p}^{2-^{2} / p}(\Omega) \times B_{q, p}^{2-{ }^{2} / p}(\Omega)$.

The next result proves the sought-after uniform stabilization of the localized feedback $\boldsymbol{w}$-system (5.3) or (5.4); that is, that the s.c. analytic semigroup $e^{\mathbb{A}_{F, q} t}$ on $\boldsymbol{W}_{\sigma}^{q}(\Omega), t \geq 0$ or on $\boldsymbol{V}_{b}^{q, p}(\Omega)$, as guaranteed by Theorem 6.1, is uniformly stable.

Theorem 6.2. Under the same setting of Theorem 4.2 concerning in particular the choice of the vectors $\boldsymbol{q}_{k}, \boldsymbol{p}_{k}, \boldsymbol{u}_{k}, f_{k}$, the $\boldsymbol{w}$-problem (5.3), (5.4) in feedback from is uniformly stable, with a decay rate $\gamma>$ 0, Re $\lambda_{N+1}<-\gamma<0$ either in $\boldsymbol{W}_{\sigma}^{q}(\Omega) \equiv \boldsymbol{L}_{\sigma}^{q}(\Omega) \times L^{q}(\Omega)$ or else in $\boldsymbol{V}_{b}^{q, p}(\Omega) \equiv \widetilde{\boldsymbol{B}}_{q, p}^{2-{ }^{2} / p}(\Omega) \times B_{q, p}^{2-{ }^{2} / p}(\Omega)$.

$$
\left\|\left[\begin{array}{l}
\boldsymbol{w}_{f} \\
w_{h}
\end{array}\right]\right\|_{(\cdot)} \leq C_{\gamma_{0}} e^{-\gamma_{0} t}\left\|\left[\begin{array}{l}
\boldsymbol{w}_{f}(0) \\
w_{h}(0)
\end{array}\right]\right\|_{(\cdot)}, t \geq 0 .
$$

In short, recalling the operator $\mathbb{A}_{F, q}$ in $5.3 \mathrm{~b}$

$$
\left\|e^{\mathbb{A}_{F, q} t}\right\|_{\mathcal{L}(\cdot)} \leq C_{\gamma_{0}} e^{-\gamma_{0} t}, t \geq 0
$$

Here, (.) denotes either the space $\boldsymbol{W}_{\sigma}^{q}(\Omega)$ or the space $\boldsymbol{V}_{b}^{q, p}(\Omega)$, see Remark 1.3.

Proof. The proof is similar to that of [62, making critical use of Theorem 4.2 on the uniform stabilization

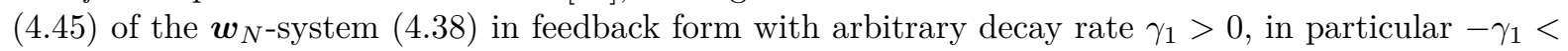
$R e \lambda_{N+1}<0$. Next, one examines the impact of the constructive feedback controls in (4.42), (44.43) and (4.46) on the $\zeta_{N}$-dynamics (3.6b), whose explicit solution is given by the variation of parameter formula

$$
\zeta_{N}(t)=e^{\mathbb{A}_{q, N}^{s}} \zeta_{N}(0)+\int_{0}^{t} e^{\mathbb{A}_{q, N}^{s}(t-\tau)}\left(I-P_{N}\right)\left[\begin{array}{c}
P_{q}\left(m \boldsymbol{u}_{N}\right)(\tau) \\
\mathcal{B}_{q} D v_{N}(\tau)
\end{array}\right] d \tau
$$

where

$$
\left\|e^{\mathbb{A}_{q, N}^{s}}\right\|_{\mathcal{L}(\cdot)} \leq C_{\gamma_{0}} e^{-\gamma_{0} t}, 0 \geq t, 0<\gamma_{0}<\left|\operatorname{Re} \lambda_{N+1}\right|
$$

while $\boldsymbol{u}_{N}(t), v_{N}(t)$ decay with an arbitrary large exponential rate $\gamma_{1}>0$ as in (4.45). The validity of (6.9) on $\boldsymbol{W}_{\sigma}^{q}(\Omega)$ follows by $(3.3)$ and (3.4), $\mathbb{A}_{q, N}^{s}$ being the generator of a s.c. analytic semigroup $e^{\mathbb{A}_{q} t}$ (Theorem 1.2), restricted to the stable subspace $\left(\boldsymbol{W}_{\sigma}^{q}\right)_{N}^{s}$ in (3.2). The validity of $\left([6.9)\right.$ on $\boldsymbol{V}_{b}^{q, p}(\Omega)$ follows by interpolation as $\mathcal{D}\left(\mathbb{A}_{q}\right)=\mathcal{D}\left(\mathcal{A}_{q}\right) \times \mathcal{D}\left(\mathcal{B}_{q}\right)=\mathcal{D}\left(A_{q}\right) \times \mathcal{D}\left(B_{q}\right)$, see (1.34).

Details are in [62, Section 10]. 


\section{Maximal $L^{p}$-regularity on $\boldsymbol{W}_{\sigma}^{q}(\Omega)$ of the linearized feedback operator $\mathbb{A}_{F, q}$ in (5.3b) up to $T=\infty$.}

Consider the following abstract dynamics

$$
\begin{gathered}
\zeta_{t}=\mathbb{A}_{F, q} \zeta+\chi, \quad \zeta(0)=\zeta_{0}, \quad \text { in } \boldsymbol{W}_{\sigma}^{q}(\Omega) \\
\zeta(t)=e^{\mathbb{A}_{F, q}} \zeta_{0}+\int_{0}^{t} e^{\mathbb{A}_{F, q}(t-s)} \chi(s) d s
\end{gathered}
$$

The main result of the present section is

Theorem 7.1. With reference to the $\boldsymbol{w}$-problem (5.3) in feedback form defining the feedback operator $\mathbb{A}_{F, q}$, assume the setting of Theorem 4.2 regarding the choice of the vectors $\boldsymbol{q}_{k}, \boldsymbol{p}_{k}, \boldsymbol{u}_{k}, f_{k}$, in (5.1), (5.2), so that Theorem 6.2 holds true. Then the operator $\mathbb{A}_{F, q}$ has maximal Lp-regularity on $\boldsymbol{W}_{\sigma}^{q}(\Omega)$ up to $T=\infty$;

$$
\mathbb{A}_{F, q} \in M \operatorname{Reg}\left(L^{p}\left(0, \infty ; \boldsymbol{W}_{\sigma}^{q}(\Omega)\right)\right)
$$

That is,

$$
(L \chi)(t)=\int_{0}^{t} e^{\mathbb{A}_{F, q}(t-s)} \chi(s) d s
$$

continuous:

$$
L^{p}\left(0, \infty ; \boldsymbol{W}_{\sigma}^{q}(\Omega)\right) \longrightarrow L^{p}\left(0, \infty ; \mathcal{D}\left(\mathbb{A}_{F, q}\right)\right)
$$

so that continuously from (7.1) with $\zeta_{0}=0$ :

$$
\begin{aligned}
& \chi \in L^{p}\left(0, \infty ; \boldsymbol{W}_{\sigma}^{q}(\Omega)\right) \longrightarrow \zeta \in \boldsymbol{X}_{p, q, \sigma}^{\infty} \times X_{p, q}^{\infty} \\
& \in L^{p}\left(0, \infty ; \mathcal{D}\left(\mathbb{A}_{F, q}\right)\right) \cap W^{1, p}\left(0, \infty ; \boldsymbol{W}_{\sigma}^{q}(\Omega)\right) \\
& \longrightarrow \boldsymbol{X}_{p, q}^{\infty} \times X_{p, q}^{\infty} \equiv L^{p}\left(0, \infty ; \boldsymbol{W}^{2, q}(\Omega)\right) \times L^{p}\left(0, \infty ; W^{2, q}(\Omega)\right)
\end{aligned}
$$

Proof. Again, as in the proof of Theorem 6.1 about analyticity of $e^{\mathbb{A}_{F, q} t}$, it suffices to show that the streamlined operator $\hat{\mathbb{A}}_{F, q}$ in (5.4), (5.5) has maximal $L^{p}$-regularity on $\boldsymbol{W}_{\sigma}^{q}(\Omega)$ up to $T<\infty$. Equivalently, since $\boldsymbol{W}_{\sigma}^{q}(\Omega)$ is reflexive, that the adjoint operator $\hat{\mathbb{A}}_{F, q}^{*}$ has maximal $L^{p}$-regularity on $\left(\boldsymbol{W}_{\sigma}^{q}(\Omega)\right)^{*}=\boldsymbol{W}_{\sigma}^{q^{\prime}}(\Omega)$ up to $T<\infty$. To establish this, we return to estimate (6.6) showing statement (6.3), with $\theta_{0}=1-\gamma=1-1 / 2 q+\varepsilon<1$. Now we invoke that $\mathbb{A}_{q}^{*}$, hence $\hat{\mathbb{A}}_{q}^{*}$ in (6.2) has maximal $L^{p}$. regularity on $\boldsymbol{W}_{\sigma}^{q^{\prime}}(\Omega)$, (by duality on Theorem $1.2(\mathrm{ii})$, since $\boldsymbol{W}_{\sigma}^{q}(\Omega)$ is a reflexive space.) We then apply known perturbation theory [23, Theorem 6.2, p311], or [53, Remark 1i, p 426 for $\beta=1$ ] to conclude that $\hat{\mathbb{A}}_{F, q}^{*} \in M \operatorname{Reg}\left(L^{p}\left(0, T ; \boldsymbol{W}_{\sigma}^{q^{\prime}}(\Omega)\right)\right), T<\infty$, and hence $\hat{\mathbb{A}}_{F, q} \in \operatorname{MReg}\left(L^{p}\left(0, T ; \boldsymbol{W}_{\sigma}^{q}(\Omega)\right)\right), T<\infty$. Then we obtain $\mathbb{A}_{F, q} \in M \operatorname{Reg}\left(L^{p}\left(0, T ; \boldsymbol{W}_{\sigma}^{q}(\Omega)\right)\right), T<\infty$. But $e^{\mathbb{A}_{F, q}}$ is uniformly stable on $\boldsymbol{W}_{\sigma}^{q}(\Omega)$ by Theorem 6.2. Hence we obtain $\mathbb{A}_{F, q} \in \operatorname{MReg}\left(L^{p}\left(0, \infty ; \boldsymbol{W}_{\sigma}^{q}(\Omega)\right)\right)$, and Theorem 7.1 is proved.

We next examine the regularity of the term $e^{\mathbb{A}_{F, q} t} \zeta_{0}$ due to the initial condition $\zeta_{0}$ as in (7.2).

Theorem 7.2. (i) Let $1<p<\frac{2 q^{\prime}}{2 q^{\prime}-1}, 1<q^{\prime} \leq 2,2 \leq q, \frac{1}{q}+\frac{1}{q^{\prime}}=1$. Consider the adjoint operator $\mathbb{A}_{F, q}^{*}=\hat{\mathbb{A}}_{F, q}^{*}+\Pi^{*}$ of $\mathbb{A}_{F, q}$ in (15.4), where $\mathcal{D}\left(\mathbb{A}_{F, q}^{*}\right)=\mathcal{D}\left(\hat{\mathbb{A}}_{F, q}^{*}\right)=\mathcal{D}\left(A_{q}^{*}\right) \times \mathcal{D}\left(B_{q}^{*}\right)$ by $(\underline{6.1 \mathrm{~b}})$. The adjoint s.c. analytic semigroup $e^{\mathbb{A}_{F, q}^{*}}$ on $\boldsymbol{W}_{\sigma}^{q^{\prime}}(\Omega)$ is uniformly stable, by duality on (6.7b). Then

$$
\begin{aligned}
e^{\mathbb{A}_{F, q}^{*} t}: \text { continuous } \equiv & \boldsymbol{V}_{b}^{q^{\prime}, p}(\Omega) \equiv \widetilde{\boldsymbol{B}}_{q^{\prime}, p}^{2-^{2} / p}(\Omega) \times B_{q^{\prime}, p}^{2-^{2} / p}(\Omega) \\
= & \left(\boldsymbol{L}_{\sigma}^{q^{\prime}}(\Omega), \mathcal{D}\left(A_{q}^{*}\right)\right)_{1-\frac{1}{p}, p} \times\left(L^{q^{\prime}}(\Omega), \mathcal{D}\left(B_{q}^{*}\right)\right)_{1-\frac{1}{p}, p} \\
& \longrightarrow \boldsymbol{X}_{p, q^{\prime}, \sigma}^{\infty} \times X_{p, q^{\prime}}^{\infty} \equiv L^{p}\left(0, \infty ; \mathcal{D}\left(\mathbb{A}_{F, q}^{*}\right)\right) \cap W^{1, p}\left(0, \infty ; \boldsymbol{W}_{\sigma}^{q^{\prime}}(\Omega)\right)
\end{aligned}
$$


(ii) Consider the original s.c. analytic feedback semigroup $e^{\mathbb{A}_{F, q} t}$ on $\boldsymbol{W}_{\sigma}^{q}(\Omega)$, which is uniformly stable here by (6.7b). Let $1<p<\frac{2 q}{2 q-1}, 2 \leq q$.

$$
\begin{aligned}
e^{\mathbb{A}_{F, q} t}: \text { continuous } \equiv & \boldsymbol{V}_{b}^{q, p}(\Omega) \equiv \widetilde{\boldsymbol{B}}_{q, p}^{2-2 / p}(\Omega) \times B_{q, p}^{2-{ }^{2} / p}(\Omega) \\
= & \left(\boldsymbol{L}_{\sigma}^{q}(\Omega), \mathcal{D}\left(A_{q}\right)\right)_{1-\frac{1}{p}, p} \times\left(L^{q}(\Omega), \mathcal{D}\left(B_{q}\right)\right)_{1-\frac{1}{p}, p} \\
& \longrightarrow \boldsymbol{X}_{p, q, \sigma}^{\infty} \times X_{p, q}^{\infty} \equiv L^{p}\left(0, \infty ; \mathcal{D}\left(\mathbb{A}_{F, q}\right)\right) \cap W^{1, p}\left(0, \infty ; \boldsymbol{W}_{\sigma}^{q}(\Omega)\right)
\end{aligned}
$$

Proof. The proof is similar to that in [62, Section 11].

\section{Proof of Theorem 2.2. Well-posedness on $X_{p, q, \sigma}^{\infty} \times X_{p, q}^{\infty}$ of the non-linear $\left[\begin{array}{l}z \\ h\end{array}\right]$-dynamics in feedback form.}

In this section we return to the translated non-linear $\left[\begin{array}{l}z \\ h\end{array}\right]$-dynamics (1.28) or (1.29) and apply to it the feedback control pair $\{\boldsymbol{u}, v\}$

$$
\left[\begin{array}{c}
P_{q} m(\boldsymbol{u}) \\
v
\end{array}\right]=\left[\begin{array}{c}
P_{q}\left(m\left(\sum_{k=1}^{K}\left\langle P_{N}\left[\begin{array}{c}
\boldsymbol{z} \\
h
\end{array}\right], \boldsymbol{q}_{k}\right\rangle \boldsymbol{u}_{k}\right)\right) \\
\sum_{k=1}^{K}\left\langle P_{N}\left[\begin{array}{c}
\boldsymbol{z} \\
h
\end{array}\right], \boldsymbol{p}_{k}\right\rangle f_{k}
\end{array}\right]=\left[\begin{array}{c}
J\left[\begin{array}{c}
\boldsymbol{z} \\
h
\end{array}\right] \\
F\left[\begin{array}{c}
\boldsymbol{z} \\
h
\end{array}\right]
\end{array}\right]
$$

as in (2.10) that is, of the same structure as the feedback operators $J$ and $F$ in (5.1), (5.2) identified on the RHS of the linearized $\boldsymbol{w}=\left[\begin{array}{l}\boldsymbol{w}_{f} \\ w_{h}\end{array}\right]$-dynamics (5.3). These are the feedback operators which produced the s.c. analytic, uniformly stable semigroup $e^{\mathbb{A}_{F, q} t}$ on $\boldsymbol{W}_{\sigma}^{q}(\Omega)=\boldsymbol{L}_{\sigma}^{q}(\Omega) \times L^{q}(\Omega)$ or on $\boldsymbol{V}_{b}^{q, p}(\Omega) \equiv \widetilde{\boldsymbol{B}}_{q, p}^{2-{ }^{2} / p}(\Omega) \times B_{q, p}^{2-{ }^{2} / p}(\Omega)$, (Theorem 6.1 and Theorem 6.2) possessing $L^{p}$-maximal regularity on $\boldsymbol{W}_{\sigma}^{q}(\Omega)$ up to $T=\infty$; (Theorem [7.1). Thus, returning to (1.28) or (1.29), in this section we consider the following feedback nonlinear problem, see (2.12)

$$
\begin{aligned}
& \frac{d}{d t}\left[\begin{array}{l}
\boldsymbol{z} \\
h
\end{array}\right]=\mathbb{A}_{F, q}\left[\begin{array}{l}
\boldsymbol{z} \\
h
\end{array}\right]-\left[\begin{array}{cc}
\mathcal{N}_{q} & 0 \\
0 & \mathcal{M}_{q}[\boldsymbol{z}]
\end{array}\right]\left[\begin{array}{l}
\boldsymbol{z} \\
h
\end{array}\right] ; \quad \mathbb{A}_{F, q}\left[\begin{array}{l}
\boldsymbol{z} \\
h
\end{array}\right]=\left[\begin{array}{cc}
\mathcal{A}_{q} & -\mathcal{C}_{\gamma} \\
-\mathcal{C}_{\theta_{e}} & 0
\end{array}\right]\left[\begin{array}{l}
\boldsymbol{z} \\
h
\end{array}\right] \\
& +\left[\begin{array}{c}
J\left[\begin{array}{l}
\boldsymbol{z} \\
h
\end{array}\right] \\
-\mathcal{B}_{q}\left(h-D F\left[\begin{array}{l}
\boldsymbol{z} \\
h
\end{array}\right]\right)
\end{array}\right],
\end{aligned}
$$

specifically as in (2.11)

$$
\left\{\begin{aligned}
\frac{d \boldsymbol{z}}{d t}-\mathcal{A}_{q} \boldsymbol{z}+\mathcal{C}_{\gamma} h+\mathcal{N}_{q} \boldsymbol{z} & =P_{q}\left(m\left(\sum_{k=1}^{K}\left\langle P_{N}\left[\begin{array}{l}
\boldsymbol{z} \\
h
\end{array}\right], \boldsymbol{q}_{k}\right\rangle \boldsymbol{u}_{k}\right)\right) \\
\frac{d h}{d t}+\mathcal{C}_{\theta_{e}} \boldsymbol{z}+\mathcal{M}_{q}[\boldsymbol{z}] h & =-\mathcal{B}_{q}\left(h-D\left(\sum_{k=1}^{K}\left\langle P_{N}\left[\begin{array}{l}
\boldsymbol{z} \\
h
\end{array}\right], \boldsymbol{p}_{k}\right\rangle f_{k}\right)\right) .
\end{aligned}\right.
$$

The variation of parameter formula for $\mathrm{Eq}(8.2)$ is

$$
\left[\begin{array}{l}
\boldsymbol{z} \\
h
\end{array}\right](t)=e^{\mathbb{A}_{F, q} t}\left[\begin{array}{c}
\boldsymbol{z}_{0} \\
h_{0}
\end{array}\right]-\int_{0}^{t} e^{\mathbb{A}_{F, q}(t-\tau)}\left[\begin{array}{c}
\mathcal{N}_{q} \boldsymbol{z}(\tau) \\
\mathcal{M}_{q}[\boldsymbol{z}] h(\tau)
\end{array}\right] d \tau .
$$


We already know from (2.7) or (6.7b) that for $\left\{\boldsymbol{z}_{0}, h_{0}\right\} \in \widetilde{\boldsymbol{B}}_{q, p}^{2-^{2} / p}(\Omega) \times B_{q, p}^{2-{ }^{2} / p}(\Omega) \equiv \boldsymbol{V}_{b}^{q, p}(\Omega), 1<p<$ $\frac{2 q}{2 q-1}$, we have: there is $M_{\gamma_{0}}$ such that

$$
\left\|e^{\mathbb{A}_{F, q} t}\left[\begin{array}{c}
\boldsymbol{z}_{0} \\
h_{0}
\end{array}\right]\right\|_{\boldsymbol{V}_{b}^{q, p}(\Omega)} \leq M_{\gamma_{0}} e^{-\gamma_{0} t}\left\|\left[\begin{array}{c}
\boldsymbol{z}_{0} \\
h_{0}
\end{array}\right]\right\|_{\boldsymbol{V}_{b}^{q, p}(\Omega)}, t \geq 0
$$

with $M_{\gamma_{0}}$ possibly depending on $p, q$. Maximal $L^{p}$-regularity properties corresponding to the solution operator formula (8.4) were established in Theorem [7.1, Theorem 7.2, Accordingly, for

$$
\begin{aligned}
& \boldsymbol{b}_{0} \equiv\left\{\boldsymbol{z}_{0}, h_{0}\right\} \in \widetilde{\boldsymbol{B}}_{q, p}^{2-{ }^{2} / p}(\Omega) \times B_{q, p}^{2-{ }^{2} / p}(\Omega) \equiv \boldsymbol{V}_{b}^{q, p}(\Omega) \\
& \boldsymbol{f} \equiv\left\{\boldsymbol{f}_{1}, f_{2}\right\} \in \boldsymbol{X}_{p, q, \sigma}^{\infty} \times X_{p, q}^{\infty} \\
& \equiv L^{p}\left(0, \infty, \mathcal{D}\left(\mathbb{A}_{F, q}\right)\right) \cap W^{1, p}\left(0, \infty ; \boldsymbol{W}_{\sigma}^{q}(\Omega)\right)(\text { see }(2.15)) \\
& \mathcal{D}\left(\mathbb{A}_{F, q}\right) \subset \mathcal{D}\left(A_{q}\right) \times W^{2, q}(\Omega) \\
& =\left[\boldsymbol{W}^{2, q}(\Omega) \cap \boldsymbol{W}_{0}^{1, q}(\Omega) \cap \boldsymbol{L}_{\sigma}^{q}(\Omega)\right] \times W^{2, q}(\Omega)(\text { see }(\underline{5.5 \mathrm{~b}})) \\
& \boldsymbol{W}_{\sigma}^{q}(\Omega)=\boldsymbol{L}_{\sigma}^{q}(\Omega) \times L^{q}(\Omega)(\text { see (1.30) }) \\
& \boldsymbol{X}_{p, q}^{\infty} \equiv L^{p}\left(0, \infty ;\left(\boldsymbol{W}^{2, q}(\Omega) \cap \boldsymbol{W}_{0}^{1, q}(\Omega) \cap \boldsymbol{L}_{\sigma}^{q}(\Omega)\right) \times W^{2, q}(\Omega)\right) \\
& \cap W^{1, p}\left(0, \infty ; \boldsymbol{W}_{\sigma}^{q}(\Omega)\right) ; \\
& X_{p, q}^{\infty} \equiv L^{p}\left(0, \infty ; W^{2, q}(\Omega) \cap W_{0}^{1, q}(\Omega)\right) \cap W^{1, q}\left(0, \infty ; L^{q}(\Omega)\right),
\end{aligned}
$$

as in (8.4), we define the operator

$$
\mathcal{F}\left(\boldsymbol{b}_{0}, \boldsymbol{f}\right) \equiv e^{\mathbb{A}_{F, q} t} \boldsymbol{b}_{0}-\int_{0}^{t} e^{\mathbb{A}_{F, q}(t-\tau)}\left[\begin{array}{c}
\mathcal{N}_{q} \boldsymbol{f}_{1}(\tau) \\
\mathcal{M}_{q}\left[\boldsymbol{f}_{1}\right] f_{2}(\tau)
\end{array}\right] d \tau
$$

The main result of this section is Theorem 2.2 restated as

Theorem 8.1. Let $d=2,3, q>d, 1<p<\frac{2 q}{2 q-1}$. There exists a positive constant $r_{1}>0$ (identified in the proof below in (8.31) such that if

$$
\left\|\boldsymbol{b}_{0}\right\|_{\boldsymbol{V}^{q, p}(\Omega)}=\left\|\left\{\boldsymbol{z}_{0}, h_{0}\right\}\right\|_{\widetilde{\boldsymbol{B}}_{q, p}^{2-2 / p}(\Omega) \times B_{q, p}^{2-2 / p}(\Omega)}<r_{1},
$$

then the operator $\mathcal{F}$ in (8.10) has a unique fixed point non-linear semigroup solution in $\boldsymbol{X}_{p, q, \sigma}^{\infty} \times X_{p, q}^{\infty}$, see (2.15) -(2.18), or (8.7)

$$
\mathcal{F}\left(\left[\begin{array}{l}
\boldsymbol{z}_{0} \\
h_{0}
\end{array}\right],\left[\begin{array}{l}
\boldsymbol{z} \\
h
\end{array}\right]\right)=\left[\begin{array}{l}
\boldsymbol{z} \\
h
\end{array}\right] \text {, or }\left[\begin{array}{l}
\boldsymbol{z} \\
h
\end{array}\right](t)=e^{\mathbb{A}_{F, q} t}\left[\begin{array}{l}
\boldsymbol{z}_{0} \\
h_{0}
\end{array}\right]-\int_{0}^{t} e^{\mathbb{A}_{F, q}(t-\tau)}\left[\begin{array}{c}
\mathcal{N}_{q} \boldsymbol{z}(\tau) \\
\mathcal{M}_{q}[\boldsymbol{z}] h(\tau)
\end{array}\right] d \tau,
$$

which therefore is the unique solution of problem (8.2) $=$ (8.3) in $\boldsymbol{X}_{p, q, \sigma}^{\infty} \times X_{p, q}^{\infty}$.

The proof of Theorem $2.2=$ Theorem 8.1 is accomplished in two steps.

Step 1:

Theorem 8.2. Let $d=2,3, q>d$ and $1<p<\frac{2 q}{2 q-1}$. There exists a positive constant $r_{1}>0$ (identified in the proof below in 88.31)) and a subsequent constant $r>0$ (identified in the proof below in (8.29)) depending on $r_{1}>0$ and a constant $C$ in (8.28), such that with $\left\|\boldsymbol{b}_{0}\right\|_{\boldsymbol{V}^{q, p}(\Omega)}<r_{1}$ as in (8.11), the operator $\mathcal{F}\left(\boldsymbol{b}_{0}, \boldsymbol{f}\right)$ in (8.10) maps a ball $B(0, r)$ in $\boldsymbol{X}_{p, q, \sigma}^{\infty} \times X_{p, q}^{\infty}$ into itself. 
Theorem 8.1 will follow then from Theorem 8.2 after establishing that

Step 2:

Theorem 8.3. Let $d=2,3, q>d$ and $1<p<\frac{2 q}{2 q-1}$. There exists a positive constant $r_{1}>0$ such that if $\left\|\boldsymbol{b}_{0}\right\|_{\boldsymbol{V}^{q, p}(\Omega)}<r_{1}$ as in (8.11), there exists a constant $0<\rho_{0}<1$ (identified in (8.56)), such that the operator $\mathcal{F}\left(\boldsymbol{b}_{0}, \boldsymbol{f}\right)$ in (8.10) defines a contraction in the ball $B\left(0, \rho_{0}\right)$ of $\boldsymbol{X}_{p, q, \sigma}^{\infty} \times X_{p, q}^{\infty}$.

The Banach contraction principle then establishes Theorem 8.1 once we prove Theorems 8.2 and 8.3 . These are proved below.

\section{Proof of Theorem 8.2}

Step 1: We start from the definition (8.10) of $\mathcal{F}\left(\boldsymbol{b}_{0}, \boldsymbol{f}\right)$ and invoke the maximal regularity properties (7.8) of Theorem 7.2 for $e^{\mathbb{A}_{F, q} t}$ and (77.6) $=(2.15)$ of Theorem 7.1 for the integral term in (8.10). We then obtain from (8.10)

$$
\begin{aligned}
&\left\|\mathcal{F}\left(\boldsymbol{b}_{0}, \boldsymbol{f}\right)\right\|_{\boldsymbol{X}_{p, q, \sigma}^{\infty} \times X_{p, q}^{\infty}} \leq\left\|e^{\mathbb{A}_{F, q} t} \boldsymbol{b}_{0}\right\|_{\boldsymbol{X}_{p, q, \sigma}^{\infty} \times X_{p, q}^{\infty}} \\
&+\left\|\int_{0}^{t} e^{\mathbb{A}_{F, q}(t-\tau)}\left[\begin{array}{c}
\mathcal{N}_{q} \boldsymbol{f}_{1}(\tau) \\
\mathcal{M}_{q}\left[\boldsymbol{f}_{1}\right] f_{2}(\tau)
\end{array}\right] d \tau\right\|_{\boldsymbol{X}_{p, q, \sigma}^{\infty} \times X_{p, q}^{\infty}} \\
& \leq C\left[\left\|\boldsymbol{b}_{0}\right\|_{\boldsymbol{V}_{b}^{q, p}(\Omega)}+\left\|\mathcal{N}_{q} \boldsymbol{f}_{1}\right\|_{L^{p}\left(0, \infty ; \boldsymbol{L}_{\sigma}^{q}(\Omega)\right)}+\left\|\mathcal{M}_{q}\left[\boldsymbol{f}_{1}\right] f_{2}\right\|_{L^{p}\left(0, \infty ; L^{q}(\Omega)\right)}\right] .
\end{aligned}
$$

Step 2: Regarding the term $\mathcal{N}_{q} \boldsymbol{f}_{1}$ we can invoke [60, Eq (8.19)] to obtain

$$
\left\|\mathcal{N}_{q} \boldsymbol{f}_{1}\right\|_{L^{p}\left(0, \infty ; L_{\sigma}^{q}(\Omega)\right)} \leq C\left\|\boldsymbol{f}_{1}\right\|_{\boldsymbol{X}_{p, q, \sigma}^{\infty}}^{2}, \boldsymbol{f}_{1} \in \boldsymbol{X}_{p, q, \sigma}^{\infty} .
$$

Regarding the term $\mathcal{M}_{q}\left[\boldsymbol{f}_{1}\right] f_{2}$, we can trace the proof in [60, from (8.10) $\longrightarrow$ (8.18)] (which yielded estimate (8.14)). For the sake of clarity, we shall reproduce the computations in the present case with $\mathcal{M}_{q}\left[\boldsymbol{f}_{1}\right] f_{2}=\boldsymbol{f}_{1} \cdot \nabla f_{2}$, see (1.20), mutatis mutandis. We shall obtain

$$
\left\|\mathcal{M}_{q}\left[\boldsymbol{f}_{1}\right] f_{2}\right\|_{L^{p}\left(0, \infty ; L^{q}(\Omega)\right)} \leq C\left\|\boldsymbol{f}_{1}\right\|_{\boldsymbol{X}_{p, q, \sigma}^{\infty}}\left\|f_{2}\right\|_{X_{p, q}^{\infty}}, \boldsymbol{f}_{1} \in \boldsymbol{X}_{p, q, \sigma}^{\infty}, f_{2} \in X_{p, q}^{\infty} .
$$

In fact, let us compute from (1.20)

$$
\begin{aligned}
\left\|\mathcal{M}_{q}\left[\boldsymbol{f}_{1}\right] f_{2}\right\|_{L^{p}\left(0, \infty ; L^{q}(\Omega)\right.}^{p} & \leq \int_{0}^{\infty}\left\|\boldsymbol{f}_{1} \cdot \nabla f_{2}\right\|_{L^{q}(\Omega)}^{p} d t \\
& \leq \int_{0}^{\infty}\left\{\int_{\Omega}\left|\boldsymbol{f}_{1}(t, x)\right|^{q}\left|\nabla f_{2}(t, x)\right|^{q} d \Omega\right\}^{p / q} d t \\
\leq \int_{0}^{\infty}\left\{\left[\sup _{\Omega}\left|\nabla f_{2}(t, x)\right|^{q}\right]^{1 / q}\left[\int_{\Omega}\left|\boldsymbol{f}_{1}(t, x)\right|^{q} d \Omega\right]^{1 / q}\right\}^{p} d t & \leq \int_{0}^{\infty}\left\|\nabla f_{2}(t, \cdot)\right\|_{\boldsymbol{L}^{\infty}(\Omega)}^{p}\left\|\boldsymbol{f}_{1}(t, \cdot)\right\|_{\boldsymbol{L}_{\sigma}^{q}(\Omega)}^{p} d t \\
\leq \sup _{0 \leq t \leq \infty} & \left\|\boldsymbol{f}_{1}(t, \cdot)\right\|_{\boldsymbol{L}_{\sigma}^{q}(\Omega)}^{p} \int_{0}^{\infty}\left\|\nabla f_{2}(t, \cdot)\right\|_{\boldsymbol{L}^{\infty}(\Omega)}^{p} d t \\
& =\left\|\boldsymbol{f}_{1}\right\|_{L^{\infty}\left(0, \infty ; \boldsymbol{L}_{\sigma}^{q}(\Omega)\right)}^{p}\left\|\nabla f_{2}\right\|_{L^{p}\left(0, \infty ; \boldsymbol{L}^{\infty}(\Omega)\right)}^{p} .
\end{aligned}
$$

See also [60, Eq (8.14)]. The following embeddings hold true (see the stronger Eq (2.16)): 
(i) [32, Proposition 4.3, p 1406 with $\mu=0, s=\infty, r=q$ ] so that the required formula reduces to $1 \geq 1 / p$, as desired

$$
\begin{aligned}
\boldsymbol{f}_{1} \in \boldsymbol{X}_{p, q, \sigma}^{\infty} \hookrightarrow \boldsymbol{f}_{1} & \in L^{\infty}\left(0, \infty ; \boldsymbol{L}_{\sigma}^{q}(\Omega)\right) \\
\text { so that, }\left\|\boldsymbol{f}_{1}\right\|_{L^{\infty}\left(0, \infty ; \boldsymbol{L}_{\sigma}^{q}(\Omega)\right)} & \leq C\left\|\boldsymbol{f}_{1}\right\|_{\boldsymbol{X}_{p, q, \sigma}^{\infty}} ;
\end{aligned}
$$

(ii) [41, Theorem 2.4 .4 , p 74 requiring $C^{1}$-boundary]

$$
W^{1, q}(\Omega) \subset L^{\infty}(\Omega) \text { for } \mathrm{q}>\operatorname{dim} \Omega=d, d=2,3,
$$

so that, with $p>1, q>d$ :

$$
\begin{aligned}
\left\|\nabla f_{2}\right\|_{L^{p}\left(0, \infty ; \boldsymbol{L}^{\infty}(\Omega)\right)}^{p} & \leq C\left\|\nabla f_{2}\right\|_{L^{p}\left(0, \infty ; \boldsymbol{W}^{1, q}(\Omega)\right)}^{p} \leq C\left\|f_{2}\right\|_{L^{p}\left(0, \infty ; W^{2, q}(\Omega)\right)}^{p} \\
& \leq C\left\|f_{2}\right\|_{X_{p, q}^{\infty}}^{p} .
\end{aligned}
$$

In going from (8.25) to (8.26) we have recalled the definition of $f_{2} \in X_{p, q}^{\infty}$ in (8.9b) or (2.15), as $f_{2}$ was taken at the outset in $\mathcal{D}\left(\mathcal{B}_{q}\right) \subset L^{q}(\Omega)$. Then the sought-after final estimate (8.16) of the nonlinear term $\mathcal{M}_{q}\left[\boldsymbol{f}_{1}\right] f_{2}$ is obtained from substituting (8.23b) and (8.26) into the RHS of (8.22).

Step 3: Substituting estimates (8.15) and (8.16) on the RHS of (8.14), we finally obtain

$$
\| \mathcal{F}\left(\boldsymbol{b}_{0}, \boldsymbol{f} \|_{\boldsymbol{X}_{p, q, \sigma}^{\infty} \times X_{p, q}^{\infty}} \leq C\left\{\left\|\boldsymbol{b}_{0}\right\|_{\boldsymbol{V}_{b}^{q, p}(\Omega)}+\left\|\boldsymbol{f}_{1}\right\|_{\boldsymbol{X}_{p, q, \sigma}^{\infty}}\left(\left\|\boldsymbol{f}_{1}\right\|_{\boldsymbol{X}_{p, q, \sigma}^{\infty}}+\left\|f_{2}\right\|_{X_{p, q}^{\infty}}\right)\right\} .\right.
$$

See [60, Eqt (8.20)].

Step 4: We now impose restrictions on the data on the RHS of (8.27): $\boldsymbol{b}_{0}$ is in a ball of radius $r_{1}>0$ in $\boldsymbol{V}_{b}^{q, p}(\Omega)=\widetilde{\boldsymbol{B}}_{q, p}^{2-^{2} / p}(\Omega) \times B_{q, p}^{2-2 / p}(\Omega)$ and $\boldsymbol{f}=\left\{\boldsymbol{f}_{1}, f_{2}\right\}$ lies in a ball of radius $r>0$ in $\boldsymbol{X}_{p, q, \sigma}^{\infty} \times X_{p, q}^{\infty}$. We further demand that the final result $\mathcal{F}\left(\boldsymbol{b}_{0}, \boldsymbol{f}\right)$ shall lie in a ball of radius $r>0$ in $\boldsymbol{X}_{p, q, \sigma}^{\infty} \times X_{p, q}^{\infty}$. Thus, we obtain from (8.27)

$$
\begin{aligned}
\left\|\mathcal{F}\left(\boldsymbol{b}_{0}, \boldsymbol{f}\right)\right\|_{\boldsymbol{X}_{p, q, \sigma}^{\infty} \times X_{p, q}^{\infty}} & \leq C\left\{\left\|\boldsymbol{b}_{0}\right\|_{\boldsymbol{V}_{b}^{q, p}(\Omega)}+\left\|\boldsymbol{f}_{1}\right\|_{\boldsymbol{X}_{p, q, \sigma}^{\infty}}\left(\left\|\boldsymbol{f}_{1}\right\|_{\boldsymbol{X}_{p, q, \sigma}^{\infty}}+\left\|f_{2}\right\|_{X_{p, q}^{\infty}}\right)\right\} \\
& \leq C\left(r_{1}+r \cdot r\right) \leq r .
\end{aligned}
$$

This implies

$$
C r^{2}-r+C r_{1} \leq 0 \quad \text { or } \quad \frac{1-\sqrt{1-4 C^{2} r_{1}}}{2 C} \leq r \leq \frac{1+\sqrt{1-4 C^{2} r_{1}}}{2 C}
$$

whereby

$$
\{\text { range of values of } \mathrm{r}\} \longrightarrow \text { interval }\left[0, \frac{1}{C}\right] \text {, as } r_{1} \searrow 0,
$$

a constraint which is guaranteed by taking

$$
r_{1} \leq \frac{1}{4 C^{2}}, C \text { being the constant in (8.28) }\left(\text { w.l.o.g. } C>\frac{1}{4}\right) .
$$

We have thus established that by taking $r_{1}$ as in (8.31) and subsequently $r$ as in (8.29), then the map

$$
\begin{aligned}
& \mathcal{F}\left(\boldsymbol{b}_{0}, \boldsymbol{f}\right) \text { takes: }\left\{\begin{array}{c}
\text { ball in } \boldsymbol{V}_{b}^{q, p}(\Omega) \\
\text { of radius } r_{1}
\end{array}\right\} \times\left\{\begin{array}{c}
\text { ball in } \boldsymbol{X}_{p, q, \sigma}^{\infty} \times X_{p, q}^{\infty} \\
\text { of radius } r
\end{array}\right\} \text { into } \\
&\left\{\begin{array}{c}
\text { ball in } \boldsymbol{X}_{p, q, \sigma}^{\infty} \times X_{p, q}^{\infty} \\
\text { of radius } r
\end{array}\right\}, d<q, 1<p<\frac{2 q}{2 q-1} .
\end{aligned}
$$


This establishes Theorem 8.2

\section{Proof of Theorem 8.3}

Step 1: For $\boldsymbol{f}=\left\{\boldsymbol{f}_{1}, f_{2}\right\}, \boldsymbol{g}=\left\{\boldsymbol{g}_{1}, g_{2}\right\}$ both in the ball of $\boldsymbol{X}_{p, q, \sigma}^{\infty} \times X_{p, q}^{\infty}$ of radius $r$ obtained in the proof of Theorem 8.2, we estimate from (8.10):

$$
\begin{aligned}
& \left\|\mathcal{F}\left(\boldsymbol{b}_{0}, \boldsymbol{f}\right)-\mathcal{F}\left(\boldsymbol{b}_{0}, \boldsymbol{g}\right)\right\|_{\boldsymbol{X}_{p, q, \sigma}^{\infty} \times X_{p, q}^{\infty}}= \\
& \left\|\int_{0}^{t} e^{\mathbb{A}_{F, q}(t-\tau)}\left[\begin{array}{c}
\mathcal{N}_{q} \boldsymbol{f}_{1}(\tau)-\mathcal{N}_{q} \boldsymbol{g}_{1}(\tau) \\
\mathcal{M}_{q}\left[\boldsymbol{f}_{1}\right] f_{2}(\tau)-\mathcal{M}_{q}\left[\boldsymbol{g}_{1}\right] g_{2}(\tau)
\end{array}\right] d \tau\right\|_{\boldsymbol{X}_{p, q, \sigma}^{\infty} \times X_{p, q}^{\infty}} \\
& \leq \widetilde{m}\left[\left\|\mathcal{N}_{q} \boldsymbol{f}_{1}-\mathcal{N}_{q} \boldsymbol{g}_{1}\right\|_{L^{p}\left(0, \infty ; \boldsymbol{L}_{\sigma}^{q}(\Omega)\right)}+\left\|\mathcal{M}_{q}\left[\boldsymbol{f}_{1}\right] f_{2}-\mathcal{M}_{q}\left[\boldsymbol{g}_{1}\right] g_{2}\right\|_{L^{p}\left(0, \infty ; L^{q}(\Omega)\right)}\right]
\end{aligned}
$$

after invoking the maximal regularity property (7.6) of Theorem 7.1 as in (8.14).

Step 2: As to the first term of the RHS of (8.33), we can invoke [60, Eq (8.41)] and obtain

$$
\begin{aligned}
& \left\|\mathcal{N}_{q} \boldsymbol{f}_{1}-\mathcal{N}_{q} \boldsymbol{g}_{1}\right\|_{L^{p}\left(0, \infty ; \boldsymbol{L}_{\sigma}^{q}(\Omega)\right)} \leq 2^{1 / p} C^{1 / p}\left\|\boldsymbol{f}_{1}-\boldsymbol{g}_{1}\right\|_{\boldsymbol{X}_{p, q, \sigma}^{\infty}}\left(\left\|\boldsymbol{f}_{1}\right\|_{\boldsymbol{X}_{p, q, \sigma}^{\infty}}\right. \\
& \left.+\left\|\boldsymbol{g}_{1}\right\|_{\boldsymbol{X}_{p, q, \sigma}^{\infty}}\right)
\end{aligned}
$$

Regarding the second term on the RHS of (8.33) involving $\mathcal{M}_{q}$, we can track the proof of [60, from (8.28) to (8.41)] (which yielded estimate (8.34)) mutatis mutandis. For the sake of clarity, we shall reproduce the computations in the present case, recalling from (1.22) that $\mathcal{M}_{q}\left[\boldsymbol{f}_{1}\right] f_{2}=\boldsymbol{f}_{1} \cdot \nabla f_{2}, \mathcal{M}_{q}\left[\boldsymbol{g}_{1}\right] g_{2}=\boldsymbol{g}_{1} \cdot \nabla g_{2}$. We shall obtain

$$
\begin{aligned}
\left\|\mathcal{M}_{q}\left[\boldsymbol{f}_{1}\right] f_{2}-\mathcal{M}_{q}\left[\boldsymbol{g}_{1}\right] g_{2}\right\|_{L^{p}\left(0, \infty ; L^{q}(\Omega)\right.}^{p} \leq C\left\{\left\|\boldsymbol{f}_{1}-\boldsymbol{g}_{1}\right\|_{\boldsymbol{X}_{p, q, \sigma}^{\infty}}^{p}\left\|f_{2}\right\|_{X_{p, q}^{\infty}}^{p}\right. & \left.+\left\|\boldsymbol{g}_{1}\right\|_{\boldsymbol{X}_{p, q, \sigma}^{\infty}}^{p}\left\|f_{2}-g_{2}\right\|_{X_{p, q}^{\infty}}^{p}\right\}
\end{aligned}
$$

In fact, adding and subtracting

$$
\begin{aligned}
\mathcal{M}_{q}\left[\boldsymbol{f}_{1}\right] f_{2}-\mathcal{M}_{q}\left[\boldsymbol{g}_{1}\right] g_{2} & =\boldsymbol{f}_{1} \cdot \nabla f_{2}-\boldsymbol{g}_{1} \cdot \nabla g_{2} \\
& =\boldsymbol{f}_{1} \cdot \nabla f_{2}-\boldsymbol{g}_{1} \cdot \nabla f_{2}+\boldsymbol{g}_{1} \cdot \nabla f_{2}-\boldsymbol{g}_{1} \cdot \nabla g_{2} \\
& =\left(\boldsymbol{f}_{1}-\boldsymbol{g}_{1}\right) \cdot \nabla f_{2}+\boldsymbol{g}_{1} \cdot \nabla\left(f_{2}-g_{2}\right)=A+B
\end{aligned}
$$

Thus, using (*) : $|A+B|^{p} \leq 2^{p}\left[|A|^{p}+|B|^{p}\right]$ [82, p. 12], we estimate

$$
\begin{aligned}
\left\|\mathcal{M}_{q}\left[\boldsymbol{f}_{1}\right] f_{2}-\mathcal{M}_{q}\left[\boldsymbol{g}_{1}\right] g_{2}\right\|_{L^{p}\left(0, \infty ; L^{q}(\Omega)\right)}^{p} & =\int_{0}^{\infty}\left\{\left[\int_{\Omega}\left|\boldsymbol{f}_{1} \cdot \nabla f_{2}-\boldsymbol{g}_{1} \cdot \nabla g_{2}\right|^{q} d \Omega\right]^{1 / q}\right\}^{p} d t \\
(\operatorname{by}(\underline{8.36)})) & =\int_{0}^{\infty}\left[\int_{\Omega}|A+B|^{q} d \Omega\right]^{p / q} d t \\
& \leq 2^{p} \int_{0}^{\infty}\left\{\int_{\Omega}\left[|A|^{q}+|B|^{q}\right] d \Omega\right\}^{p / q} d t \\
& =2^{p} \int_{0}^{\infty}\left\{\left[\int_{\Omega}|A|^{q} d \Omega+\int|B|^{q} d \Omega\right]^{1 / q}\right\}^{p} d t \\
& =2^{p} \int_{0}^{\infty}\left\{\left[\|A\|_{L^{q}(\Omega)}^{q}+\|B\|_{L^{q}(\Omega)}^{q}\right]^{1 / q}\right\}^{p} d t
\end{aligned}
$$




$$
\begin{aligned}
\left(\text { by }(*) \text { with } p \rightarrow \frac{1}{q}\right) \quad & \leq 2^{p} \cdot 2^{p / q} \int_{0}^{\infty}\left\{\|A\|_{L^{q}(\Omega)}+\|B\|_{L^{q}(\Omega)}\right\}^{p} d t \\
(\text { by }(*)) \quad \leq & \leq 2^{p+p+^{p} / q} \int_{0}^{\infty}\left[\|A\|_{L^{q}(\Omega)}^{p}+\|B\|_{L^{q}(\Omega)}^{p}\right] d t \\
& =2^{p+p+^{p} / q} \int_{0}^{\infty}\left[\left\|\left(\boldsymbol{f}_{1}-\boldsymbol{g}_{1}\right) \cdot \nabla f_{2}\right\|_{L^{q}(\Omega)}^{p}\right. \\
& \left.\quad+\left\|\boldsymbol{g}_{1} \cdot \nabla\left(f_{2}-g_{2}\right)\right\|_{L^{q}(\Omega)}^{p}\right] d t \\
& \leq 2^{p+p+{ }^{p} / q} \int_{0}^{\infty}\left\{\left\|\boldsymbol{f}_{1}-\boldsymbol{g}_{1}\right\|_{\boldsymbol{L}_{\sigma}^{q}(\Omega)}^{p}\left\|\nabla f_{2}\right\|_{L^{q}(\Omega)}^{p}\right. \\
& \left.+\left\|\boldsymbol{g}_{1}\right\|_{\boldsymbol{L}_{\sigma}^{q}(\Omega)}^{p}\left\|\nabla\left(f_{2}-g_{2}\right)\right\|_{L^{q}(\Omega)}^{p}\right\} d t,
\end{aligned}
$$

recalling the definitions of $A$ and $B$ in (8.36) in passing from (8.43) to (8.44). 60, This is the counterpart Eq (8.36)]. Next we proceed by majorizing the $L^{q}(\Omega)$-norm for $\nabla f_{2}$ and $\nabla\left(f_{2}-g_{2}\right)$ by the $L^{\infty}(\Omega)$-norm. We obtain

$$
\begin{aligned}
&\left\|\mathcal{M}_{q}\left[\boldsymbol{f}_{1}\right] f_{2}-\mathcal{M}_{q}\left[\boldsymbol{g}_{1}\right] g_{2}\right\|_{L^{p}\left(0, \infty ; L^{q}(\Omega)\right.}^{p} \leq \text { RHS of (8.45) } \\
& \leq c 2^{p+p+^{p} / q}\left\{\sup _{0 \leq t \leq \infty}\left\|\boldsymbol{f}_{1}-\boldsymbol{g}_{1}\right\|_{\boldsymbol{L}_{\sigma}^{q}(\Omega)}^{p} \int_{0}^{\infty}\left\|\nabla f_{2}\right\|_{L^{\infty}(\Omega)}^{p}\right. \\
&\left.\quad+\sup _{0 \leq t \leq \infty}\left\|\boldsymbol{g}_{1}\right\|_{\boldsymbol{L}_{\sigma}^{q}(\Omega)}^{p} \int_{0}^{\infty}\left\|\nabla\left(f_{2}-g_{2}\right)\right\|_{L^{\infty}(\Omega)}^{p} d t\right\} \\
& \leq c 2^{p+p+^{p} / q}\left\{\left\|\boldsymbol{f}_{1}-\boldsymbol{g}_{1}\right\|_{L^{\infty}\left(0, \infty ; \boldsymbol{L}_{\sigma}^{q}(\Omega)\right)}^{p}\left\|\nabla f_{2}\right\|_{L^{p}\left(0, \infty ; L^{\infty}(\Omega)\right)}^{p}\right. \\
&\left.+\left\|\boldsymbol{g}_{1}\right\|_{L^{\infty}\left(0, \infty ; \boldsymbol{L}_{\sigma}^{q}(\Omega)\right)}^{p}\left\|\nabla\left(f_{2}-g_{2}\right)\right\|_{L^{p}\left(0, \infty ; L^{\infty}(\Omega)\right)}^{p}\right\}
\end{aligned}
$$

by (8.23b) and (8.26)

$$
\leq C\left\{\left\|\boldsymbol{f}_{1}-\boldsymbol{g}_{1}\right\|_{\boldsymbol{X}_{p, q, \sigma}^{\infty}}^{p}\left\|f_{2}\right\|_{X_{p, q}^{\infty}}^{p}+\left\|\boldsymbol{g}_{1}\right\|_{\boldsymbol{X}_{p, q, \sigma}^{\infty}}^{p}\left\|f_{2}-g_{2}\right\|_{X_{p, q}^{\infty}}^{p}\right\},
$$

counterpart of [60, Eq (8.39)].

Step 3: We substitute estimate (8.34) and estimate (8.48) on the RHS of (8.33) and obtain via $(*)$

$$
\begin{array}{r}
\left\|\mathcal{F}\left(\boldsymbol{b}_{0}, \boldsymbol{f}\right)-\mathcal{F}\left(\boldsymbol{b}_{0}, \boldsymbol{g}\right)\right\|_{\boldsymbol{X}_{p, q, \sigma}^{\infty} \times X_{p, q}^{\infty}}^{p} \leq 2^{p} \widetilde{m}^{p}\left\{\left\|\mathcal{N}_{q} \boldsymbol{f}_{1}-\mathcal{N}_{q} \boldsymbol{g}_{1}\right\|_{L^{p}\left(0, \infty ; L^{q}(\Omega)\right)}^{p}\right. \\
\left.+\left\|\mathcal{M}_{q}\left[\boldsymbol{f}_{1}\right] f_{2}-\mathcal{M}_{q}\left[\boldsymbol{g}_{1}\right] g_{2}\right\|_{L^{p}\left(0, \infty ; L^{q}(\Omega)\right)}^{p}\right\} \\
\leq C_{p} \widetilde{m}^{p}\left\{\left\|\boldsymbol{f}_{1}-\boldsymbol{g}_{1}\right\|_{\boldsymbol{X}_{p, q, \sigma}^{\infty}}^{p}\left(\left\|\boldsymbol{f}_{1}\right\|_{\boldsymbol{X}_{p, q, \sigma}^{\infty}}+\left\|\boldsymbol{g}_{1}\right\|_{\boldsymbol{X}_{p, q, \sigma}^{\infty}}\right)^{p}\right. \\
\left.+\left\|\boldsymbol{f}_{1}-\boldsymbol{g}_{1}\right\|_{\boldsymbol{X}_{p, q, \sigma}^{\infty}}^{p}\left\|f_{2}\right\|_{X_{p, q}^{\infty}}^{p}+\left\|\boldsymbol{g}_{1}\right\|_{\boldsymbol{X}_{p, q, \sigma}^{\infty}}^{p}\left\|f_{2}-g_{2}\right\|_{X_{p, q}^{\infty}}^{p}\right\} .
\end{array}
$$

This is the counterpart of [60, Eq (8.42)].

Step 4: Next pick the points $\boldsymbol{f}=\left\{\boldsymbol{f}_{1}, f_{2}\right\}$ and $\boldsymbol{g}=\left\{\boldsymbol{g}_{1}, g_{2}\right\}$ in a ball of $\boldsymbol{X}_{p, q, \sigma}^{\infty} \times X_{p, q}^{\infty}$ of radius $R$ :

$$
\begin{aligned}
\|\boldsymbol{f}\|_{\boldsymbol{X}_{p, q, \sigma}^{\infty} \times X_{p, q}^{\infty}} & =\left\|\boldsymbol{f}_{1}\right\|_{\boldsymbol{X}_{p, q, \sigma}^{\infty}}+\left\|f_{2}\right\|_{X_{p, q}^{\infty}}<R \\
\|\boldsymbol{g}\|_{\boldsymbol{X}_{p, q, \sigma}^{\infty} \times X_{p, q}^{\infty}} & =\left\|\boldsymbol{g}_{1}\right\|_{\boldsymbol{X}_{p, q, \sigma}^{\infty}}+\left\|g_{2}\right\|_{X_{p, q}^{\infty}}<R .
\end{aligned}
$$


Then (8.51), (8.52) used (8.50) implies

$$
\begin{gathered}
\left\|\mathcal{F}\left(\boldsymbol{b}_{0}, \boldsymbol{f}\right)-\mathcal{F}\left(\boldsymbol{b}_{0}, \boldsymbol{g}\right)\right\|_{\boldsymbol{X}_{p, q, \sigma}^{\infty} \times X_{p, q}^{\infty}}^{p} \leq C_{p}\left\{\left\|\boldsymbol{f}_{1}-\boldsymbol{g}_{1}\right\|_{\boldsymbol{X}_{p, q, \sigma}^{\infty}}^{p}\left[(2 R)^{p}+R^{p}\right]\right. \\
\left.+\left\|f_{2}-g_{2}\right\|_{X_{p, q}^{\infty}}^{p} R^{p}\right\} \\
\leq C_{p}\left\{\left\|\boldsymbol{f}_{1}-\boldsymbol{g}_{1}\right\|_{\boldsymbol{X}_{p, q, \sigma}^{\infty}}^{p}\left[\left(2^{p}+1\right) R^{p}\right]\right. \\
\left.+\left\|f_{2}-g_{2}\right\|_{X_{p, q}^{\infty}}^{p} R^{p}\right\} \\
\left(K_{p}=C_{p}\left(2^{p}+1\right)\right) \\
\leq K_{p} R^{p}\left\{\left\|\boldsymbol{f}_{1}-\boldsymbol{g}_{1}\right\|_{\boldsymbol{X}_{p, q, \sigma}^{\infty}}^{p}+\left\|f_{2}-g_{2}\right\|_{X_{p, q}^{\infty}}^{p}\right\} \\
{\left[a>0, b>0, a^{p}+b^{p} \leq(a+b)^{p}\right]} \\
\leq K_{p} R^{p}\left\{\left\|\boldsymbol{f}_{1}-\boldsymbol{g}_{1}\right\|_{\boldsymbol{X}_{p, q, \sigma}^{\infty}}+\left\|f_{2}-g_{2}\right\|_{X_{p, q}^{\infty}}\right\}^{p}
\end{gathered}
$$

using $K_{p}=C_{p}\left(2^{p}+1\right)$ and $a^{p}+b^{p} \leq(a+b)^{p}$ for $a>0, b>0$. Finally,

$$
\begin{aligned}
\left\|\mathcal{F}\left(\boldsymbol{b}_{0}, \boldsymbol{f}\right)-\mathcal{F}\left(\boldsymbol{b}_{0}, \boldsymbol{g}\right)\right\|_{\boldsymbol{X}_{p, q, \sigma}^{\infty} \times X_{p, q}^{\infty}} & \leq K_{p}^{1 / p} R\left\{\left\|\boldsymbol{f}_{1}-\boldsymbol{g}_{1}\right\|_{\boldsymbol{X}_{p, q, \sigma}^{\infty}}+\left\|f_{2}-g_{2}\right\|_{X_{p, q}^{\infty}}\right\} \\
& =\rho_{0}\|\boldsymbol{f}-\boldsymbol{g}\|_{\boldsymbol{X}_{p, q, \sigma}^{\infty} \times X_{p, q}^{\infty}}
\end{aligned}
$$

and $\mathcal{F}\left(\boldsymbol{b}_{0}, \boldsymbol{f}\right)$ is a contraction on the space $\boldsymbol{X}_{p, q, \sigma}^{\infty} \times X_{p, q}^{\infty}$ as soon as

$$
\rho_{0}=K_{p}^{1 / p} R<1 \quad \text { or } \quad R<\frac{1}{K_{p}^{1 / p}} .
$$

In this case, the map $\mathcal{F}\left(\boldsymbol{b}_{0}, \boldsymbol{f}\right)$ defined in (8.10) has a fixed point $\left[\begin{array}{l}\boldsymbol{z} \\ h\end{array}\right]$ in $\boldsymbol{X}_{p, q, \sigma}^{\infty} \times X_{p, q}^{\infty}$ :

$$
\mathcal{F}\left(\boldsymbol{b}_{0},\left[\begin{array}{c}
\boldsymbol{z} \\
h
\end{array}\right]\right)=\left[\begin{array}{c}
\boldsymbol{z} \\
h
\end{array}\right] \text {, or }\left[\begin{array}{c}
\boldsymbol{z} \\
h
\end{array}\right](t)=e^{\mathbb{A}_{F, q} t}\left[\begin{array}{c}
\boldsymbol{z}_{0} \\
h_{0}
\end{array}\right]-\int_{0}^{t} e^{\mathbb{A}_{F, q}(t-\tau)}\left[\begin{array}{c}
\mathcal{N}_{q} \boldsymbol{z}(\tau) \\
\mathcal{M}_{q}[\boldsymbol{z}] h(\tau)
\end{array}\right] d \tau,
$$

and such point $\left[\begin{array}{l}z \\ h\end{array}\right]$ is the unique solution of the translated non-linear system (8.2), or (8.3), with finite dimensional control

$$
\left[\begin{array}{c}
P_{q} m(\boldsymbol{u}) \\
v
\end{array}\right]=\left[\begin{array}{c}
P_{q}\left(m\left(\sum_{k=1}^{K}\left(P_{N}\left[\begin{array}{c}
\boldsymbol{z} \\
h
\end{array}\right], \boldsymbol{p}_{k}\right) \boldsymbol{u}_{k}\right)\right) \\
\sum_{k=1}^{K}\left(P_{N}\left[\begin{array}{c}
\boldsymbol{z} \\
h
\end{array}\right], \boldsymbol{p}_{k}\right) f_{k}
\end{array}\right]
$$

in feedback form, as described by Eq (8.1). Theorem 8.3 and hence Theorem 8.1 are proved.

Remark 8.1. Recall from (2.1) and the statement of Theorem 2.1 that $\boldsymbol{u}_{k} \in\left(\widehat{\boldsymbol{W}}_{\sigma}^{q}\right)_{N}^{u} \subset \widehat{\boldsymbol{L}}_{\sigma}^{q}(\Omega)$ as established in Appendix C] This means that the feedback control acting on the fluid variable $\boldsymbol{u}$ is of reduced dimension $(d-1)$ to include the $d^{\text {th }}$ component.

\section{Proof of Theorem 2.3: local exponential decay of the non- linear $[z, h]$ translated dynamics $(2.11)=(2.12)$ with finite dimen- sional localized feedback controls and $(d-1)$ dimensional $\boldsymbol{u}_{K}$.}

In this section we return to the feedback problem (2.12), rewritten equivalently as in (2.14)

$$
\left[\begin{array}{c}
\boldsymbol{z} \\
h
\end{array}\right](t)=e^{\mathbb{A}_{F, q} t}\left[\begin{array}{c}
\boldsymbol{z}_{0} \\
h_{0}
\end{array}\right]-\int_{0}^{t} e^{\mathbb{A}_{F, q}(t-\tau)}\left[\begin{array}{c}
\mathcal{N}_{q} \boldsymbol{z}(\tau) \\
\mathcal{M}_{q}[\boldsymbol{z}] h(\tau)
\end{array}\right] d \tau
$$


For $\boldsymbol{b}_{0}=\left[\boldsymbol{z}_{0}, h_{0}\right]$ in a small ball of $\boldsymbol{V}_{b}^{q, p}(\Omega)=\widetilde{\boldsymbol{B}}_{q, p}^{2-^{2} / p}(\Omega) \times B_{q, p}^{2-{ }^{2} / p}(\Omega)$, Theorem $[8.1$ ( $=$ Theorem [2.2) provides a unique solution $\{\boldsymbol{z}, h\}$ in a small ball of $\boldsymbol{X}_{p, q, \sigma}^{\infty} \times X_{p, q}^{\alpha}$. We recall from (6.7) of Theorem 6.2

$$
\left\|e^{\mathbb{A}_{F, q} t}\left[\begin{array}{c}
\boldsymbol{z}_{0} \\
h_{0}
\end{array}\right]\right\|_{\boldsymbol{V}_{b}^{q, p}(\Omega)} \leq M_{\gamma_{0}} e^{-\gamma_{0} t}\left\|\left[\begin{array}{c}
\boldsymbol{z}_{0} \\
h_{0}
\end{array}\right]\right\|_{\boldsymbol{V}_{b}^{q, p}(\Omega)}, t \geq 0
$$

$\boldsymbol{V}_{b}^{q, p}(\Omega)=\widetilde{\boldsymbol{B}}_{q, p}^{2-2 / p}(\Omega) \times B_{q, p}^{2-2 / p}(\Omega)$. Our goal is to show that for $\left[\boldsymbol{z}_{0}, h_{0}\right]$ in a small ball of $\boldsymbol{V}_{b}^{q, p}(\Omega)$, problem (9.1) satisfies the exponential decay

$$
\left\|\left[\begin{array}{l}
\boldsymbol{z} \\
h
\end{array}\right](t)\right\|_{\boldsymbol{V}_{b}^{q, p}(\Omega)} \leq C_{a} e^{-a t}\left\|\left[\begin{array}{l}
\boldsymbol{z}_{0} \\
h_{0}
\end{array}\right]\right\|_{\boldsymbol{V}_{b}^{q, p}(\Omega)}, t \geq 0, \quad \text { for some constants } a>0, C=C_{a} \geq 1 .
$$

Step 1: Starting from (9.1) and using the decay (9.2), we estimate

$$
\begin{aligned}
& \left\|\left[\begin{array}{c}
\boldsymbol{z}(t) \\
h(t)
\end{array}\right]\right\|_{\boldsymbol{V}_{b}^{q, p}(\Omega)} \leq M_{\gamma_{0}} e^{-\gamma_{0} t}\left\|\left[\begin{array}{c}
\boldsymbol{z}_{0} \\
h_{0}
\end{array}\right]\right\|_{\boldsymbol{V}_{b}^{q, p}(\Omega)}+ \\
& \sup _{0 \leq t \leq \infty}\left\|\int_{0}^{t} e^{\mathbb{A}_{F, q}(t-\tau)}\left[\begin{array}{c}
\mathcal{N}_{q} \boldsymbol{z}(\tau) \\
\mathcal{M}_{q}[\boldsymbol{z}] h(\tau)
\end{array}\right] d \tau\right\|_{\boldsymbol{V}_{b}^{q, p}(\Omega)} \\
& \leq M_{\gamma_{0}} e^{-\gamma_{0} t}\left\|\left[\begin{array}{c}
\boldsymbol{z}_{0} \\
h_{0}
\end{array}\right]\right\|_{\boldsymbol{V}_{b}^{q, p}(\Omega)}+C_{1}\left\|\int_{0}^{t} e^{\mathbb{A}_{F, q}(t-\tau)}\left[\begin{array}{c}
\mathcal{N}_{q} \boldsymbol{z}(\tau) \\
\mathcal{M}_{q}[\boldsymbol{z}] h(\tau)
\end{array}\right] d \tau\right\|_{\boldsymbol{X}_{p, q, \sigma}^{\infty} \times X_{p, q}^{\infty}} \\
& \leq M_{\gamma_{0}} e^{-\gamma_{0} t}\left\|\left[\begin{array}{c}
\boldsymbol{z}_{0} \\
h_{0}
\end{array}\right]\right\|_{\boldsymbol{V}_{b}^{q, p}(\Omega)}+C_{2}\left[\left\|\mathcal{N}_{q} \boldsymbol{z}\right\|_{L^{p}\left(0, \infty ; \boldsymbol{L}_{\sigma}^{q}(\Omega)\right)}+\right. \\
& \left.\left\|\mathcal{M}_{q}[\boldsymbol{z}] h\right\|_{L^{p}\left(0, \infty ; L^{q}(\Omega)\right)}\right] \\
& \leq M_{\gamma_{0}} e^{-\gamma_{0} t}\left\|\left[\begin{array}{c}
\boldsymbol{z}_{0} \\
h_{0}
\end{array}\right]\right\|_{\boldsymbol{V}_{b}^{q, p}(\Omega)}+C_{3}\left[\|\boldsymbol{z}\|_{\boldsymbol{X}_{p, q, \sigma}^{\infty}}^{2}+\|\boldsymbol{z}\|_{\boldsymbol{X}_{p, q, \sigma}^{\infty}}\|h\|_{X_{p, q}^{\infty}}\right] .
\end{aligned}
$$

In going from (9.4) to (9.5) we have recalled the embedding $\boldsymbol{X}_{p, q, \sigma}^{\infty} \times X_{p, q}^{\infty} \hookrightarrow L^{\infty}\left(0, \infty ; \boldsymbol{V}_{b}^{q, p}(\Omega)\right)$ from (2.16)-(2.18). Next, in going from (9.5) to (9.6) we have used the maximal regularity property (7.6) of Theorem 7.1. Finally, to go from (9.6) to (9.7), we have invoked (8.15) for $\mathcal{N}_{q} \boldsymbol{z}$ and (8.16) for $\mathcal{M}_{q}[\boldsymbol{z}] h$. Thus, the conclusion of Step 1 is

$$
\begin{aligned}
& \left\|\left[\begin{array}{c}
\boldsymbol{z}(t) \\
h(t)
\end{array}\right]\right\|_{\boldsymbol{V}_{b}^{q, p}(\Omega)} \leq M_{\gamma_{0}} e^{-\gamma_{0} t}\left\|\left[\begin{array}{c}
\boldsymbol{z}_{0} \\
h_{0}
\end{array}\right]\right\|_{\boldsymbol{V}_{b}^{q, p}(\Omega)}+C_{3}\|\boldsymbol{z}\|_{\boldsymbol{X}_{p, q, \sigma}^{\infty}}\left[\|\boldsymbol{z}\|_{\boldsymbol{X}_{p, q, \sigma}^{\infty}}+\|h\|_{X_{p, q}^{\infty}}\right] \\
& =M_{\gamma_{0}} e^{-\gamma_{0} t}\left\|\left[\begin{array}{c}
\boldsymbol{z}_{0} \\
h_{0}
\end{array}\right]\right\|_{\boldsymbol{V}_{b}^{q, p}(\Omega)}+C_{3}\|\boldsymbol{z}\|_{\boldsymbol{X}_{p, q, \sigma}^{\infty}}\|[\boldsymbol{z}, h]\|_{\boldsymbol{X}_{p, q, \sigma}^{\infty} \times X_{p, q}^{\infty}} .
\end{aligned}
$$

Step 2: We now return to (9.1) and take the $\boldsymbol{X}_{p, q, \sigma}^{\infty} \times X_{p, q}^{\infty}$ norm across:

$$
\begin{aligned}
&\left\|\left[\begin{array}{c}
\boldsymbol{z} \\
h
\end{array}\right](t)\right\|_{\boldsymbol{X}_{p, q, \sigma}^{\infty} \times X_{p, q}^{\infty}} \leq\left\|e^{\mathbb{A}_{F, q} t}\left[\begin{array}{c}
\boldsymbol{z}_{0} \\
h_{0}
\end{array}\right]\right\|_{\boldsymbol{X}_{p, q, \sigma}^{\infty} \times X_{p, q}^{\infty}}+ \\
&\left\|\int_{0}^{t} e^{\mathbb{A}_{F, q}(t-\tau)}\left[\begin{array}{c}
\mathcal{N}_{q} \boldsymbol{z}(\tau) \\
\mathcal{M}_{q}[\boldsymbol{z}] h(\tau)
\end{array}\right] d \tau\right\|_{\boldsymbol{X}_{p, q, \sigma}^{\infty} \times X_{p, q}^{\infty}}
\end{aligned}
$$




$$
\left(\operatorname{by}(17.8) \leq M_{1}\left\|\left[\begin{array}{c}
z_{0} \\
h_{0}
\end{array}\right]\right\|_{\boldsymbol{V}_{b}^{q, p}(\Omega)}+C_{3}\|\boldsymbol{z}\|_{\boldsymbol{X}_{p, q, \sigma}^{\infty}}\|[\boldsymbol{z}, h]\|_{\boldsymbol{X}_{p, q, \sigma}^{\infty} \times X_{p, q}^{\infty}}\right.
$$

by invoking (7.8) of Theorem 7.2 on the first semigroup term in (9.10) and the estimate from (9.5) to (9.9) on the second integral term in (9.10). Thus (9.11) is established and implies

$$
\left\|\left[\begin{array}{c}
\boldsymbol{z} \\
h
\end{array}\right]\right\|_{\boldsymbol{X}_{p, q, \sigma}^{\infty} \times X_{p, q}^{\infty}}\left[1-C_{3}\|\boldsymbol{z}\|_{\boldsymbol{X}_{p, q, \sigma}^{\infty}}\right] \leq M_{1}\left\|\left[\begin{array}{c}
\boldsymbol{z}_{0} \\
h_{0}
\end{array}\right]\right\|_{\boldsymbol{V}_{b}^{q, p}(\Omega)} .
$$

[This is the counterpart of [60, Eq (9.7)]].

Step 3: The well-posedness Theorem 8.1 says that

$$
\left\{\begin{array}{c}
\text { If }\left\|\left[\begin{array}{c}
\boldsymbol{z}_{0} \\
h_{0}
\end{array}\right]\right\|_{\boldsymbol{V}_{b}^{q, p}(\Omega)} \leq r_{1} \\
\text { for } r_{1} \text { sufficiently small }
\end{array}\right\} \Longrightarrow\left\{\begin{array}{c}
\text { The solution }[\boldsymbol{z}, h] \text { satisfies } \\
\left\|\left[\begin{array}{c}
\boldsymbol{z} \\
h
\end{array}\right]\right\|_{\boldsymbol{X}_{p, q, \sigma}^{\infty} \times X_{p, q}^{\infty}} \leq r
\end{array}\right\}
$$

where the constant $r$ satisfies the constraint (8.29) in terms of the constant $r_{1}$ and some constant $C$ in (8.27) or (8.28) or (8.15) or (8.16). We seek to guarantee that we have

$$
\|\boldsymbol{z}\|_{\boldsymbol{X}_{p, q, \sigma}^{\infty}} \leq \frac{1}{2 C_{3}}<\frac{1}{2 C}, \text { hence } \frac{1}{2} \leq\left[1-C_{3}\|\boldsymbol{z}\|_{\boldsymbol{X}_{p, q, \sigma}^{\infty}}\right],
$$

where we can always take the constant $C_{3}$ in (9.14) greater than the constant $C$ in (8.29), (8.30), (8.31). Then (9.14) can be achieved by choosing $r_{1}>0$ sufficiently small. In fact, as $r_{1} \searrow 0$. Eq. (8.30) shows that the interval $r_{\min } \leq r \leq r_{\max }$ of corresponding values of $r$ tends to the interval $\left[0, \frac{1}{C}\right]$. Thus, (9.14) can be achieved as $r_{\min } \searrow 0: 0<r_{\min }<r<\frac{1}{2 C}$. Next (9.14) implies that (9.12) becomes

$$
\left\|\left[\begin{array}{c}
\boldsymbol{z} \\
h
\end{array}\right]\right\|_{\boldsymbol{X}_{p, q, \sigma}^{\infty} \times X_{p, q}^{\infty}} \leq 2 M_{1}\left\|\left[\begin{array}{l}
\boldsymbol{z}_{0} \\
h_{0}
\end{array}\right]\right\|_{\boldsymbol{V}_{b}^{q, p}(\Omega)} \leq 2 M_{1} r_{1} .
$$

by (9.13). Substituting (9.15) in estimate (9.9) then yields

$$
\begin{aligned}
\left\|\left[\begin{array}{c}
\boldsymbol{z}(t) \\
h(t)
\end{array}\right]\right\|_{\boldsymbol{V}_{b}^{q, p}(\Omega)} & \leq M_{\gamma_{0}} e^{-\gamma_{0} t}\left\|\left[\begin{array}{c}
\boldsymbol{z}_{0} \\
h_{0}
\end{array}\right]\right\|_{\boldsymbol{V}_{b}^{q, p}(\Omega)}+2 M_{1} C_{3}\|\boldsymbol{z}\|_{\boldsymbol{X}_{p, q, \sigma}^{\infty}}\left\|\left[\begin{array}{l}
\boldsymbol{z}_{0} \\
h_{0}
\end{array}\right]\right\|_{\boldsymbol{V}_{b}^{q, p}(\Omega)} \\
& \leq \widehat{M}\left[e^{-\gamma_{0} t}+4 \widehat{M} C_{3} r_{1}\right]\left\|\left[\begin{array}{c}
\boldsymbol{z}_{0} \\
h_{0}
\end{array}\right]\right\|_{\boldsymbol{V}_{b}^{q, p}(\Omega)}
\end{aligned}
$$

again by (9.15) with $\widehat{M}=\max \left\{M_{\gamma_{0}}, M_{1}\right\}$. This is the counterpart of [60, Eq (9.16)].

Step 4: We now take $T$ sufficiently large and $r_{1}>0$ sufficiently small so that

$$
\beta=\widehat{M}\left[e^{-\gamma_{0} T}+4 \widehat{M} C_{3} r_{1}\right]<1 .
$$

Then (9.16) implies

$$
\left\|\left[\begin{array}{l}
\boldsymbol{z}(T) \\
h(T)
\end{array}\right]\right\|_{\boldsymbol{V}_{b}^{q, p}(\Omega)} \leq \beta\left\|\left[\begin{array}{l}
\boldsymbol{z}_{0} \\
h_{0}
\end{array}\right]\right\|_{\boldsymbol{V}_{b}^{q, p}(\Omega)}
$$

$\boldsymbol{V}_{b}^{q, p}(\Omega)=\widetilde{\boldsymbol{B}}_{q, p}^{2-2 / p}(\Omega) \times B_{q, p}^{2-2 / p}(\Omega)$, and hence

$$
\left\|\left[\begin{array}{c}
\boldsymbol{z}(n T) \\
h(n T)
\end{array}\right]\right\|_{\boldsymbol{V}_{b}^{q, p}(\Omega)} \leq \beta\left\|\left[\begin{array}{c}
\boldsymbol{z}((n-1) T) \\
h((n-1) T)
\end{array}\right]\right\|_{\boldsymbol{V}_{b}^{q, p}(\Omega)} \leq \beta^{n}\left\|\left[\begin{array}{c}
\boldsymbol{z}_{0} \\
h_{0}
\end{array}\right]\right\|_{\boldsymbol{V}_{b}^{q, p}(\Omega)} .
$$


Since $\beta<1$, the semigroup property of the evolution implies [6] that there are constants $M_{\widetilde{\gamma}} \geq 1, \widetilde{\gamma}>0$ such that

$$
\left\|\left[\begin{array}{c}
\boldsymbol{z}(t) \\
h(t)
\end{array}\right]\right\|_{\boldsymbol{V}_{b}^{q, p}(\Omega)} \leq M_{\widetilde{\gamma}} e^{-\widetilde{\gamma} t}\left\|\left[\begin{array}{c}
\boldsymbol{z}_{0} \\
h_{0}
\end{array}\right]\right\|_{\boldsymbol{V}_{b}^{q, p}(\Omega)}
$$

with $\left\|\left[\boldsymbol{z}_{0}, h_{0}\right]\right\|_{\boldsymbol{V}_{b}^{q, p}(\Omega)} \leq r_{1}=$ small. This proves (2.18), i.e Theorem 2.3 ,

Remark 9.1. The above computations - (9.17) through (9.19) - can be used to support qualitatively the intuitive expectation that "the larger the decay rate $\gamma_{0}$ in (6.7) $=(9.2)$ of the linearized feedback $\boldsymbol{w}$-dynamics (5.4), the larger the decay rate $\widetilde{\gamma}$ in (9.20) of the nonlinear feedback $\{\boldsymbol{z}, h\}$-dynamics (9.1) or (2.13); hence the larger the rate $\widetilde{\gamma}$ in (9.20) of the original $\{\boldsymbol{y}, \theta\}$-dynamics in (1.1)".

The following considerations are somewhat qualitative. Let $S(t)$ denote the non-linear semigroup in the space $\boldsymbol{V}_{b}^{q, p}(\Omega) \equiv \widetilde{\boldsymbol{B}}_{q, p}^{2-^{2} / p}(\Omega) \times B_{q, p}^{2-2 / p}(\Omega)$, with infinitesimal generator $\left[\mathbb{A}_{F, q}-\mathcal{N}_{q}\right]$ describing the feedback $\{\boldsymbol{z}, h\}$-dynamics, (9.1) or (2.13) as guaranteed by the well posedness Theorem $2.2=$ Theorem 8.1. Thus, $\left[\begin{array}{l}\boldsymbol{z}(t) \\ h(t)\end{array}\right]=S(t)\left[\begin{array}{l}\boldsymbol{z}_{0} \\ h_{0}\end{array}\right]$ on $\boldsymbol{V}_{b}^{q, p}(\Omega)$. By (9.17), we can rewrite (9.18) as:

$$
\|S(T)\|_{\mathcal{L}\left(\boldsymbol{V}_{b}^{q, p}(\Omega)\right)} \leq \beta<1 .
$$

It follows from [6, p 178] via the semigroup property that

$$
-\widetilde{\gamma} \text { is just below } \frac{\ln \beta}{T}<0 .
$$

Pick $r_{1}>0$ in (9.17) so small that $4 \widehat{M}^{2} C_{3} r_{1}$ is negligible, so that $\beta$ is just above $\widehat{M} e^{-\gamma_{0} T}$, so $\ln \beta$ is just above $\left[\ln \widehat{M}-\gamma_{0} T\right]$, hence

$$
\frac{\ln \beta}{T} \text { is just above }\left[\left(-\gamma_{0}\right)+\frac{\ln \widehat{M}}{T}\right] \text {. }
$$

Hence, by (9.22), (9.23),

$$
\widetilde{\gamma} \sim \gamma_{0}-\frac{\ln \widehat{M}}{T}
$$

and the larger $\gamma_{0}$, the larger is $\widetilde{\gamma}$, as desired.

\section{A Some auxiliary results for the Stokes and Oseen operators: analytic semigroup generation, maximal regularity, domains of fractional powers.}

In this section we collect some known results used in the paper. As a prerequisite of the present Appendix A. we make reference to the paragraph Definition of Besov spaces $\boldsymbol{B}_{q, p}^{s}$ on domains of class $C^{1}$ as real interpolation of Sobolev spaces, Eqts (1.7)-(1.11) and Remark 1.2.

(a) The Stokes and Oseen operators generate a strongly continuous analytic semigroup on $\boldsymbol{L}_{\sigma}^{q}(\Omega), 1<q<\infty$.

Theorem A.1. Let $d \geq 2,1<q<\infty$ and let $\Omega$ be a bounded domain in $\mathbb{R}^{d}$ of class $C^{3}$. Then

(i) the Stokes operator $-A_{q}=P_{q} \Delta$ in (1.14), repeated here as

$$
-A_{q} \psi=P_{q} \Delta \psi, \quad \psi \in \mathcal{D}\left(A_{q}\right)=\boldsymbol{W}^{2, q}(\Omega) \cap \boldsymbol{W}_{0}^{1, q}(\Omega) \cap \boldsymbol{L}_{\sigma}^{q}(\Omega)
$$

generates a s.c. analytic semigroup $e^{-A_{q} t}$ on $\boldsymbol{L}_{\sigma}^{q}(\Omega)$. See [33] and the review paper [37, Theorem 2.8 .5 p 17]. 
(ii) The Oseen operator $\mathcal{A}_{q}$ in (1.17)

$$
\mathcal{A}_{q}=-\left(\nu A_{q}+A_{o, q}\right), \quad \mathcal{D}\left(\mathcal{A}_{q}\right)=\mathcal{D}\left(A_{q}\right) \subset \boldsymbol{L}_{\sigma}^{q}(\Omega)
$$

generates a s.c. analytic semigroup $e^{\mathcal{A}_{q} t}$ on $\boldsymbol{L}_{\sigma}^{q}(\Omega)$. This follows as $A_{o, q}$ is relatively bounded with respect to $A_{q}^{1 / 2}$, see (1.15): thus a standard theorem on perturbation of an analytic semigroup generator applies [68, Corollary 2.4, p 81].

(iii)

$$
\left\{\begin{array}{c}
0 \in \rho\left(A_{q}\right)=\text { the resolvent set of the Stokes operator } A_{q} \\
A_{q}^{-1}: \boldsymbol{L}_{\sigma}^{q}(\Omega) \longrightarrow \boldsymbol{L}_{\sigma}^{q}(\Omega) \text { is compact. }
\end{array}\right.
$$

(iv) The s.c. analytic Stokes semigroup $e^{-A_{q} t}$ is uniformly stable on $\boldsymbol{L}_{\sigma}^{q}(\Omega)$ : there exist constants $M \geq 1, \delta>0$ (possibly depending on $q$ ) such that

$$
\left\|e^{-A_{q} t}\right\|_{\mathcal{L}\left(\boldsymbol{L}_{\sigma}^{q}(\Omega)\right)} \leq M e^{-\delta t}, t>0 .
$$

(b) Domains of fractional powers, $\mathcal{D}\left(A_{q}^{\alpha}\right), 0<\alpha<1$ of the Stokes operator $A_{q}$ on $\boldsymbol{L}_{\sigma}^{q}(\Omega), 1<$ $q<\infty$. We elaborate on (1.16)

Theorem A.2. For the domains of fractional powers $\mathcal{D}\left(A_{q}^{\alpha}\right), 0<\alpha<1$, of the Stokes operator $A_{q}$ in (A.1) $=$ (1.14), the following complex interpolation relation holds true [34] and [37, Theorem $2.8 .5, p 18]$

$$
\left[\mathcal{D}\left(A_{q}\right), \boldsymbol{L}_{\sigma}^{q}(\Omega)\right]_{1-\alpha}=\mathcal{D}\left(A_{q}^{\alpha}\right), 0<\alpha<1,1<q<\infty
$$

in particular

$$
\left[\mathcal{D}\left(A_{q}\right), \boldsymbol{L}_{\sigma}^{q}(\Omega)\right]_{\frac{1}{2}}=\mathcal{D}\left(A_{q}^{1 / 2}\right) \equiv \boldsymbol{W}_{0}^{1, q}(\Omega) \cap \boldsymbol{L}_{\sigma}^{q}(\Omega) .
$$

Thus, on the space $\mathcal{D}\left(A_{q}^{1 / 2}\right)$, the norms

$$
\|\nabla \cdot\|_{L^{q}(\Omega)} \text { and }\|\|_{L^{q}(\Omega)}
$$

are related via Poincaré inequality.

(c) The Stokes operator $-A_{q}$ and the Oseen operator $\mathcal{A}_{q}, 1<q<\infty$ generate s.c. analytic semigroups on the Besov space, from (1.11)

$$
\begin{aligned}
\left(\boldsymbol{L}_{\sigma}^{q}(\Omega), \mathcal{D}\left(A_{q}\right)\right)_{1-\frac{1}{p}, p} & =\left\{\boldsymbol{g} \in \boldsymbol{B}_{q, p}^{2-^{2} / p}(\Omega): \operatorname{div} \boldsymbol{g}=0,\left.\boldsymbol{g}\right|_{\Gamma}=0\right\} \\
& \text { if } \frac{1}{q}<2-\frac{2}{p}<2 ; \\
\left(\boldsymbol{L}_{\sigma}^{q}(\Omega), \mathcal{D}\left(A_{q}\right)\right)_{1-\frac{1}{p}, p} & =\left\{\boldsymbol{g} \in \boldsymbol{B}_{q, p}^{2-2 / p}(\Omega): \operatorname{div} \boldsymbol{g}=0,\left.\boldsymbol{g} \cdot \nu\right|_{\Gamma}=0\right\} \\
& \equiv \widetilde{\boldsymbol{B}}_{q, p}^{2-2 / p}(\Omega), \text { if } 0<2-\frac{2}{p}<\frac{1}{q}
\end{aligned}
$$

Theorem A.1 states that the Stokes operator $-A_{q}$ generates a s.c. analytic semigroup on the space $\boldsymbol{L}_{\sigma}^{q}(\Omega), 1<q<\infty$, hence on the space $\mathcal{D}\left(A_{q}\right)$ in (1.14) $=$ (A.1), with norm $\|\cdot\|_{\mathcal{D}\left(A_{q}\right)}=\left\|A_{q} \cdot\right\|_{\boldsymbol{L}_{\sigma}^{q}(\Omega)}$ as $0 \in \rho\left(A_{q}\right)$. Then, one obtains that the Stokes operator $-A_{q}$ generates a s.c. analytic semigroup on the real interpolation spaces in (A.8). Next, the Oseen operator $\mathcal{A}_{q}=-\left(\nu A_{q}+A_{o, q}\right)$ in (A.2) $=$ (1.17) likewise generates a s.c. analytic semigroup $e^{\mathcal{A}_{q} t}$ on $\boldsymbol{L}_{\sigma}^{q}(\Omega)$ since $A_{o, q}$ is relatively bounded w.r.t. $A_{q}^{1 / 2}$, as $A_{o, q} A_{q}^{-1 / 2}$ is bounded on $\boldsymbol{L}_{\sigma}^{q}(\Omega)$. Moreover $\mathcal{A}_{q}$ generates a s.c. analytic semigroup on $\mathcal{D}\left(\mathcal{A}_{q}\right)=\mathcal{D}\left(A_{q}\right)$ (equivalent norms). Hence $\mathcal{A}_{q}$ generates a s.c. analytic semigroup on the real 
interpolation space of (A.8). Here below, however, we shall formally state the result only in the case $2-2 / p<1 / q$. i.e. $1<p<{ }^{2 q} / 2 q-1$, in the space $\widetilde{\boldsymbol{B}}_{q, p}^{2-2 / p}(\Omega)$, as this does not contain B.C., Remark 1.2. The objective of the present paper is precisely to obtain stabilization results on spaces that do not recognize B.C.

Theorem A.3. Let $1<q<\infty, 1<p<{ }^{2 q} / 2 q-1$

(i) The Stokes operator $-A_{q}$ in (A.1) $=$ (1.14) generates a s.c. analytic semigroup $e^{-A_{q} t}$ on the space $\widetilde{B}_{q, p}^{2-{ }^{2} / p}(\Omega)$ defined in (1.11) $=(\mathrm{A} .8 \mathrm{~b}$ ) which moreover is uniformly stable, as in (A.4),

$$
\left\|e^{-A_{q} t}\right\|_{\mathcal{L}\left(\widetilde{\boldsymbol{B}}_{q, p}^{2-2 / p}(\Omega)\right)} \leq M e^{-\delta t}, \quad t>0 .
$$

(ii) The Oseen operator $\mathcal{A}_{q}$ in $\left(\right.$ A.2 $=$ (1.17) generates a s.c. analytic semigroup $e^{\mathcal{A}_{q} t}$ on the space $\widetilde{\boldsymbol{B}}_{q, p}^{2-2 / p}(\Omega)$ in (1.11) $=($ A.8).

(d) Space of maximal $L^{p}$ regularity on $\boldsymbol{L}_{\sigma}^{q}(\Omega)$ of the Stokes operator $-A_{q}, 1<p<\infty, 1<$ $q<\infty$ up to $T=\infty$. We return to the dynamic Stokes problem in $\{\boldsymbol{\varphi}(t, x), \pi(t, x)\}$

$$
\left\{\begin{array}{rlrl}
\boldsymbol{\varphi}_{t}-\Delta \varphi+\nabla \pi & =\boldsymbol{F} & \text { in }(0, T] \times \Omega \equiv Q \\
\operatorname{div} \varphi & \equiv 0 & \text { in } Q \\
\left.\varphi\right|_{\Sigma} \equiv 0 & \text { in }(0, T] \times \Gamma \equiv \Sigma \\
\left.\varphi\right|_{t=0} & =\varphi_{0} & \text { in } \Omega,
\end{array}\right.
$$

rewritten in abstract form, after applying the Helmholtz projection $P_{q}$ to (A.10a) and recalling $A_{q}$ in 1 A.1. $=$ (1.14) as

$$
\boldsymbol{\varphi}^{\prime}+A_{q} \boldsymbol{\varphi}=F_{\sigma} \equiv P_{q} \boldsymbol{F}, \quad \boldsymbol{\varphi}_{0} \in\left(\boldsymbol{L}_{\sigma}^{q}(\Omega), \mathcal{D}\left(A_{q}\right)\right)_{1-\frac{1}{p}, p} .
$$

Next, we introduce the space of maximal regularity for $\left\{\boldsymbol{\varphi}, \varphi^{\prime}\right\}$ as [37, p 2; Theorem 2.8.5.iii, p 17], [32, p 1404-5], with $T$ up to $\infty$ :

$$
\widetilde{\boldsymbol{X}}_{p, q, \sigma}^{T}=L^{p}\left(0, T ; \mathcal{D}\left(A_{q}\right)\right) \cap W^{1, p}\left(0, T ; \boldsymbol{L}_{\sigma}^{q}(\Omega)\right)
$$

(recall $(\underline{\text { A.1 }})=(1.14)$ for $\left.\mathcal{D}\left(A_{q}\right)\right)$ and the corresponding space for the pressure as

$$
\tilde{Y}_{p, q}^{T}=L^{p}\left(0, T ; \widehat{W}^{1, q}(\Omega)\right), \quad \widehat{W}^{1, q}(\Omega)=W^{1, q}(\Omega) / \mathbb{R} .
$$

The following embedding, also called trace theorem, holds true [2, Theorem 4.10.2, p 180, BUC for $T=\infty]$, 69.

$$
\begin{array}{ll}
\widetilde{\boldsymbol{X}}_{p, q, \sigma}^{T} \subset \widetilde{\boldsymbol{X}}_{p, q}^{T} \equiv L^{p}\left(0, T ; \boldsymbol{W}^{2, q}(\Omega)\right) \cap W^{1, p}\left(0, T ; \boldsymbol{L}^{q}(\Omega)\right) & \\
& \hookrightarrow C\left([0, T] ; \boldsymbol{B}_{q, p}^{22^{2} / p}(\Omega)\right) .
\end{array}
$$

For a function $\boldsymbol{g}$ such that div $\boldsymbol{g} \equiv 0,\left.\boldsymbol{g}\right|_{\Gamma}=0$ we have $\boldsymbol{g} \in \widetilde{\boldsymbol{X}}_{p, q}^{T} \Longleftrightarrow \boldsymbol{g} \in \widetilde{\boldsymbol{X}}_{p, q, \sigma}^{T}$.

The solution of Eq (A.11) is

$$
\boldsymbol{\varphi}(t)=e^{-A_{q} t} \boldsymbol{\varphi}_{0}+\int_{0}^{t} e^{-A_{q}(t-s)} F_{\sigma}(\tau) d \tau .
$$

The following is the celebrated result on maximal regularity on $\boldsymbol{L}_{\sigma}^{q}(\Omega)$ of the Stokes problem due originally to Solonnikov 81] reported in [37, Theorem 2.8.5.(iii) and Theorem 2.10.1 p24 for $\varphi_{0}=0$ ], [73], [32, Proposition 4.1, p 1405]. 
Theorem A.4. Let $1<p, q<\infty, T \leq \infty$. With reference to problem (A.10) assume

$$
F_{\sigma} \in L^{p}\left(0, T ; \boldsymbol{L}_{\sigma}^{q}(\Omega)\right), \boldsymbol{\varphi}_{0} \in\left(\boldsymbol{L}_{\sigma}^{q}(\Omega), \mathcal{D}\left(A_{q}\right)\right)_{1-\frac{1}{p}, p} .
$$

Then there exists a unique solution $\varphi \in \widetilde{\boldsymbol{X}}_{p, q, \sigma}^{T}, \pi \in \widetilde{Y}_{p, q}^{T}$ to the dynamic Stokes problem (A.10) or (A.11), continuously on the data: there exist constants $C_{0}, C_{1}$ independent of $T, F_{\sigma}, \varphi_{0}$ such that via A.14

$$
\begin{aligned}
& C_{0}\|\boldsymbol{\varphi}\|_{C\left([0, T] ; \boldsymbol{B}_{q, p}^{2-2 / p}(\Omega)\right)} \leq\|\boldsymbol{\varphi}\|_{\widetilde{\boldsymbol{X}}_{p, q, \sigma}^{T}}+\|\pi\|_{\widetilde{Y}_{p, q}^{T}} \\
& \quad \equiv\left\|\boldsymbol{\varphi}^{\prime}\right\|_{L^{p}\left(0, T ; \boldsymbol{L}_{\sigma}^{q}(\Omega)\right)}+\left\|A_{q} \boldsymbol{\varphi}\right\|_{L^{p}\left(0, T ; \boldsymbol{L}_{\sigma}^{q}(\Omega)\right)}+\|\pi\|_{\widetilde{Y}_{p, q}^{T}} \\
& \leq C_{1}\left\{\left\|F_{\sigma}\right\|_{L^{p}\left(0, T ; \boldsymbol{L}_{\sigma}^{q}(\Omega)\right)}+\left\|\boldsymbol{\varphi}_{0}\right\|_{\left(\boldsymbol{L}_{\sigma}^{q}(\Omega), \mathcal{D}\left(A_{q}\right)\right)_{1-\frac{1}{p}, p}}\right\} .
\end{aligned}
$$

In particular,

(i) With reference to the variation of parameters formula (A.15) of problem (A.11) arising from the Stokes problem (A.10), we have recalling (A.12): the map

$$
\begin{gathered}
F_{\sigma} \longrightarrow \int_{0}^{t} e^{-A_{q}(t-\tau)} F_{\sigma}(\tau) d \tau \text { : continuous } \\
L^{p}\left(0, T ; \boldsymbol{L}_{\sigma}^{q}(\Omega)\right) \longrightarrow \widetilde{\boldsymbol{X}}_{p, q, \sigma}^{T} \equiv L^{p}\left(0, T ; \mathcal{D}\left(A_{q}\right)\right) \cap W^{1, p}\left(0, T ; \boldsymbol{L}_{\sigma}^{q}(\Omega)\right) .
\end{gathered}
$$

(ii) The s.c. analytic semigroup $e^{-A_{q} t}$ generated by the Stokes operator $-A_{q}($ see $(\mathrm{A} .1)=(1.14))$ on the space $\left(\boldsymbol{L}_{\sigma}^{q}(\Omega), \mathcal{D}\left(A_{q}\right)\right)_{1-\frac{1}{p}, p}$ (see statement below (A.8) satisfies

$$
\begin{aligned}
e^{-A_{q} t}: \text { continuous } & \left(\boldsymbol{L}_{\sigma}^{q}(\Omega), \mathcal{D}\left(A_{q}\right)\right)_{1-\frac{1}{p}, p} \\
& \longrightarrow \widetilde{\boldsymbol{X}}_{p, q, \sigma}^{T} \equiv \\
& L^{p}\left(0, T ; \mathcal{D}\left(A_{q}\right)\right) \cap W^{1, p}\left(0, T ; \boldsymbol{L}_{\sigma}^{q}(\Omega)\right) .
\end{aligned}
$$

In particular via (A.8b), for future use, for $1<q<\infty, 1<p<\frac{2 q}{2 q-1}$, the s.c. analytic semigroup $e^{-A_{q} t}$ on the space $\widetilde{\boldsymbol{B}}_{q, p}^{2-{ }^{2} / p}(\Omega)$, satisfies

$$
e^{-A_{q} t}: \text { continuous } \widetilde{\boldsymbol{B}}_{q, p}^{2-2 / p}(\Omega) \longrightarrow \widetilde{\boldsymbol{X}}_{p, q, \sigma}^{T}
$$

(iii) Moreover, for future use, for $1<q<\infty, 1<p<\frac{2 q}{2 q-1}$, then (A.17) specializes to

$$
\|\boldsymbol{\varphi}\|_{\widetilde{\boldsymbol{X}}_{p, q, \sigma}^{T}}+\|\pi\|_{\widetilde{Y}_{p, q}^{T}} \leq C\left\{\left\|F_{\sigma}\right\|_{L^{p}\left(0, T ; \boldsymbol{L}_{\sigma}^{q}(\Omega)\right)}+\left\|\boldsymbol{\varphi}_{0}\right\|_{\widetilde{\boldsymbol{B}}_{q, p}^{2-2 / p}(\Omega)}\right\} .
$$

(e) Maximal $L^{p}$ regularity on $L_{\sigma}^{q}(\Omega)$ of the Oseen operator $\mathcal{A}_{q}, 1<p<\infty, 1<q<\infty$, up to $T<\infty$. We next transfer the maximal regularity of the Stokes operator $\left(-A_{q}\right)$ on $\boldsymbol{L}_{\sigma}^{q}(\Omega)$-asserted in Theorem A.4 into the maximal regularity of the Oseen operator $\mathcal{A}_{q}=-\nu A_{q}-A_{o, q}$ in (A.2) exactly on the same space $\widetilde{\boldsymbol{X}}_{p, q, \sigma}^{T}$ defined in (1.12), however only up to $T<\infty$.

Thus, consider the dynamic Oseen problem in $\{\boldsymbol{\varphi}(t, x), \pi(t, x)\}$ with equilibrium solution $\boldsymbol{y}_{e}$, see (1.2):

$$
\left\{\begin{array}{rlrl}
\boldsymbol{\varphi}_{t}-\Delta \varphi+L_{e}(\boldsymbol{\varphi})+\nabla \pi & =\boldsymbol{F} & \text { in }(0, T] \times \Omega \equiv Q \\
\operatorname{div} \varphi \equiv 0 & \text { in } Q \\
\left.\varphi\right|_{\Sigma} \equiv 0 & \text { in }(0, T] \times \Gamma \equiv \Sigma \\
\left.\varphi\right|_{t=0} & =\varphi_{0} & \text { in } \Omega
\end{array}\right.
$$




$$
L_{e}(\boldsymbol{\varphi})=\left(\boldsymbol{y}_{e} . \nabla\right) \boldsymbol{\varphi}+(\boldsymbol{\varphi} \cdot \nabla) \boldsymbol{y}_{e}
$$

rewritten in abstract form, after applying the Helmholtz projector $P_{q}$ to (A.22a) and recalling $\mathcal{A}_{q}$ in (A.2), as

$$
\boldsymbol{\varphi}_{t}=\mathcal{A}_{q} \boldsymbol{\varphi}+P_{q} \boldsymbol{F}=-\nu A_{q} \boldsymbol{\varphi}-A_{o, q} \boldsymbol{\varphi}+\boldsymbol{F}_{\sigma}, \quad \boldsymbol{\varphi}_{0} \in\left(\boldsymbol{L}_{\sigma}^{q}(\Omega), \mathcal{D}\left(A_{q}\right)\right)_{1-\frac{1}{p}, p}
$$

whose solution is via $(1.18)=($ A.2

$$
\begin{gathered}
\boldsymbol{\varphi}(t)=e^{\mathcal{A}_{q} t} \boldsymbol{\varphi}_{0}+\int_{0}^{t} e^{\mathcal{A}_{q}(t-\tau)} \boldsymbol{F}_{\sigma}(\tau) d \tau \\
\boldsymbol{\varphi}(t)=e^{-\nu A_{q} t} \boldsymbol{\varphi}_{0}+\int_{0}^{t} e^{-\nu A_{q}(t-\tau)} \boldsymbol{F}_{\sigma}(\tau) d \tau-\int_{0}^{t} e^{-\nu A_{q}(t-\tau)} A_{o, q} \boldsymbol{\varphi}(\tau) d \tau .
\end{gathered}
$$

Theorem A.5. Let $1<p, q<\infty, 0<T<\infty$. Assume (as in (A.16))

$$
\boldsymbol{F}_{\sigma} \in L^{p}\left(0, T ; \boldsymbol{L}_{\sigma}^{q}(\Omega)\right), \quad \boldsymbol{\varphi}_{0} \in\left(\boldsymbol{L}_{\sigma}^{q}(\Omega), \mathcal{D}\left(A_{q}\right)\right)_{1-\frac{1}{p}, p}
$$

where $\mathcal{D}\left(A_{q}\right)=\mathcal{D}\left(\mathcal{A}_{q}\right)$, see $(\mathrm{A} .2)=(1.18)$. Then there exists a unique solution $\boldsymbol{\varphi} \in \widetilde{\boldsymbol{X}}_{p, q, \sigma}^{T}, \pi \in \widetilde{Y}_{p, q}^{T}$ of the dynamic Oseen problem (A.22), continuously on the data: that is, there exist constants $C_{0}, C_{1}$ independent of $\boldsymbol{F}_{\sigma}, \boldsymbol{\varphi}_{0}$ such that

$$
\begin{aligned}
C_{0}\|\boldsymbol{\varphi}\|_{C\left([0, T] ; \boldsymbol{B}_{q, p}^{2-2 / p}(\Omega)\right)} & \leq\|\boldsymbol{\varphi}\|_{\widetilde{\boldsymbol{X}}_{p, q, \sigma}^{T}}+\|\pi\|_{\widetilde{Y}_{p, q}^{T}} \\
& \equiv\left\|\boldsymbol{\varphi}^{\prime}\right\|_{L^{p}\left(0, T ; \boldsymbol{L}^{q}(\Omega)\right)}+\left\|A_{q} \boldsymbol{\varphi}\right\|_{L^{p}\left(0, T ; \boldsymbol{L}^{q}(\Omega)\right)}+\|\pi\|_{\widetilde{Y}_{p, q}^{T}} \\
& \leq C_{T}\left\{\left\|\boldsymbol{F}_{\sigma}\right\|_{L^{p}\left(0, T ; \boldsymbol{L}_{\sigma}^{q}(\Omega)\right)}+\left\|\boldsymbol{\varphi}_{0}\right\|_{\left(\boldsymbol{L}_{\sigma}^{q}(\Omega), \mathcal{D}\left(A_{q}\right)\right)_{1-\frac{1}{p}, p}}\right\}
\end{aligned}
$$

where $T<\infty$. Equivalently, for $1<p, q<\infty$

i. The map

$$
\begin{aligned}
& \boldsymbol{F}_{\sigma} \longrightarrow \int_{0}^{t} e^{\mathcal{A}_{q}(t-\tau)} \boldsymbol{F}_{\sigma}(\tau) d \tau: \text { continuous } \\
& L^{p}\left(0, T ; \boldsymbol{L}_{\sigma}^{q}(\Omega)\right) \longrightarrow L^{p}\left(0, T ; \mathcal{D}\left(\mathcal{A}_{q}\right)=\mathcal{D}\left(A_{q}\right)\right)
\end{aligned}
$$

where then automatically, see A.24

$$
L^{p}\left(0, T ; \boldsymbol{L}_{\sigma}^{q}(\Omega)\right) \longrightarrow W^{1, p}\left(0, T ; \boldsymbol{L}_{\sigma}^{q}(\Omega)\right)
$$

and ultimately via A.12

$$
L^{p}\left(0, T ; \boldsymbol{L}_{\sigma}^{q}(\Omega)\right) \longrightarrow \widetilde{\boldsymbol{X}}_{p, q, \sigma}^{T} \equiv L^{p}\left(0, T ; \mathcal{D}\left(A_{q}\right)\right) \cap W^{1, p}\left(0, T ; \boldsymbol{L}_{\sigma}^{q}(\Omega)\right) .
$$

ii. The s.c. analytic semigroup $e^{\mathcal{A}_{q} t}$ generated by the Oseen operator $\mathcal{A}_{q}($ see $(\mathrm{A.2})=(1.18)$ ) on the space $\left(\boldsymbol{L}_{\sigma}^{q}(\Omega), \mathcal{D}\left(A_{q}\right)\right)_{1-\frac{1}{p}, p}$ satisfies for $1<p, q<\infty$

$$
e^{\mathcal{A}_{q} t}: \text { continuous }\left(\boldsymbol{L}_{\sigma}^{q}(\Omega), \mathcal{D}\left(A_{q}\right)\right)_{1-\frac{1}{p}, p} \longrightarrow L^{p}\left(0, T ; \mathcal{D}\left(\mathcal{A}_{q}\right)=\mathcal{D}\left(A_{q}\right)\right)
$$

and hence automatically by (A.12)

$$
e^{\mathcal{A}_{q} t}: \text { continuous }\left(\boldsymbol{L}_{\sigma}^{q}(\Omega), \mathcal{D}\left(A_{q}\right)\right)_{1-\frac{1}{p}, p} \longrightarrow \widetilde{\boldsymbol{X}}_{p, q, \sigma}^{T}
$$


In particular, for future use, for $1<q<\infty, 1<p<\frac{2 q}{2 q-1}$, we have that the s.c. analytic semigroup $e^{\mathcal{A}_{q} t}$ on the space $\widetilde{\boldsymbol{B}}_{q, p}^{2-2 / p}(\Omega)$, satisfies

$$
e^{\mathcal{A}_{q} t}: \text { continuous } \widetilde{\boldsymbol{B}}_{q, p}^{2-^{2} / p}(\Omega) \longrightarrow L^{p}\left(0, T ; \mathcal{D}\left(\mathcal{A}_{q}\right)=\mathcal{D}\left(A_{q}\right)\right), T<\infty .
$$

and hence automatically

$$
e^{\mathcal{A}_{q} t}: \text { continuous } \widetilde{\boldsymbol{B}}_{q, p}^{2-^{2} / p}(\Omega) \longrightarrow \widetilde{\boldsymbol{X}}_{p, q, \sigma}^{T}, T<\infty .
$$

A proof is given in [60, Appendix B].

\section{B Material in support of the proof of Theorem 4.1: the re- quired $\mathrm{UCP}$ and $D^{*} \mathcal{B}_{q}^{*} f$ in (4.18).}

\section{B.1 The required UCP.}

We return to the operator $\mathbb{A}_{q}$ in (1.34):

$$
\begin{aligned}
\mathbb{A}_{q}=\left[\begin{array}{cc}
\mathcal{A}_{q} & -\mathcal{C}_{\gamma} \\
-\mathcal{C}_{\theta_{e}} & -\mathcal{B}_{q}
\end{array}\right]: \boldsymbol{W}_{\sigma}^{q}(\Omega)=\boldsymbol{L}_{\sigma}^{q}(\Omega) \times L^{q}(\Omega) \supset \mathcal{D}\left(\mathbb{A}_{q}\right)=\mathcal{D}\left(\mathcal{A}_{q}\right) \times \mathcal{D}\left(\mathcal{B}_{q}\right) \\
=\left(\boldsymbol{W}^{2, q}(\Omega) \cap \boldsymbol{W}_{0}^{1, q}(\Omega) \cap \boldsymbol{L}_{\sigma}^{q}(\Omega)\right) \times\left(W^{2, q}(\Omega) \cap W_{0}^{1, q}(\Omega)\right) \longrightarrow \boldsymbol{W}_{\sigma}^{q}(\Omega) .
\end{aligned}
$$

With $\boldsymbol{\Phi}=[\boldsymbol{\varphi}, \psi]$, the PDE-version of $\mathbb{A}_{q} \boldsymbol{\Phi}=\lambda \boldsymbol{\Phi}$ is

$$
\left\{\begin{aligned}
-\nu \Delta \boldsymbol{\varphi}+L_{e}(\boldsymbol{\varphi})+\nabla \pi-\gamma \psi \boldsymbol{e}_{d} & =\lambda \boldsymbol{\varphi} & & \text { in } \Omega \\
-\kappa \Delta \psi-\boldsymbol{y}_{e} \cdot \nabla \psi+\boldsymbol{\varphi} \cdot \nabla \theta_{e} & =\lambda \psi & & \text { in } \Omega \\
\operatorname{div} \boldsymbol{\varphi} & =0 & & \text { in } \Omega \\
\boldsymbol{\varphi}=0, \psi & =0 & & \text { on } \Gamma .
\end{aligned}\right.
$$

Several UCP for over-determined versions of the eigenproblem (B.2) are given in [89]. However, establishing in Theorem 4.1 controllability of the finite dimensional projected problem (4.23) or (4.29) via verification of the Kalman rank condition (4.31) involves the following UCP for the adjoint problem. First, recall from (4.4) that the adjoint $\mathbb{A}_{q}^{*}$ or $\mathbb{A}_{q}$ in (B.1) is

$$
\begin{aligned}
\mathbb{A}_{q}^{*}=\left[\begin{array}{cc}
\mathcal{A}_{q}^{*} & -\mathcal{C}_{\theta_{e}}^{*} \\
-\mathcal{C}_{\gamma}^{*} & -\mathcal{B}_{q}^{*}
\end{array}\right]: \boldsymbol{W}_{\sigma}^{q^{\prime}}(\Omega)=\boldsymbol{L}_{\sigma}^{q^{\prime}}(\Omega) \times L^{q^{\prime}}(\Omega) \supset \mathcal{D}\left(\mathbb{A}_{q}^{*}\right)=\mathcal{D}\left(\mathcal{A}_{q}^{*}\right) \times \mathcal{D}\left(\mathcal{B}_{q}^{*}\right) \\
=\left(\boldsymbol{W}^{2, q^{\prime}}(\Omega) \cap \boldsymbol{W}_{0}^{1, q^{\prime}}(\Omega) \cap \boldsymbol{L}_{\sigma}^{q^{\prime}}(\Omega)\right) \times\left(W^{2, q^{\prime}}(\Omega) \cap \boldsymbol{W}_{0}^{1, q^{\prime}}(\Omega)\right) \longrightarrow \boldsymbol{W}_{\sigma}^{q^{\prime}}(\Omega) .
\end{aligned}
$$

With $\boldsymbol{\Phi}^{*}=\left[\boldsymbol{\varphi}^{*}, \psi^{*}\right]$, the PDE-version of $\mathbb{A}_{q}^{*} \boldsymbol{\Phi}^{*}=\lambda \boldsymbol{\Phi}^{*}$ is

$$
\left\{\begin{aligned}
-\nu \Delta \boldsymbol{\varphi}^{*}+L_{e}^{*}\left(\boldsymbol{\varphi}^{*}\right)+\psi^{*} \nabla \theta_{e}+\nabla \pi & =\lambda \boldsymbol{\varphi}^{*} & & \text { in } \Omega \\
-\kappa \Delta \psi^{*}-\boldsymbol{y}_{e} \cdot \nabla \psi^{*}-\gamma \boldsymbol{\varphi}^{*} \cdot \boldsymbol{e}_{d} & =\lambda \psi^{*} & & \text { in } \Omega \\
\operatorname{div} \boldsymbol{\varphi}^{*} & =0 & & \text { in } \Omega \\
\boldsymbol{\varphi}^{*}=0, \psi^{*} & =0 & & \text { on } \Gamma .
\end{aligned}\right.
$$

The UCP invoked in the proof of Theorem 4.1 is 
Theorem B.1. [89, Theorem 5] Let $\{\boldsymbol{\varphi}, \psi, \pi\} \in\left[\boldsymbol{W}^{2, q}(\Omega) \cap \boldsymbol{L}_{\sigma}^{q}(\Omega)\right] \times W^{2, q}(\Omega), \pi \in W^{1, q}(\Omega)$, be a solution of the following dual problem

$$
\left\{\begin{aligned}
-\nu \Delta \boldsymbol{\varphi}^{*}+L_{e}^{*}\left(\boldsymbol{\varphi}^{*}\right)+\psi^{*} \nabla \theta_{e}+\nabla \pi & =\lambda \boldsymbol{\varphi}^{*} & & \text { in } \Omega \\
-\kappa \Delta \psi^{*}-\boldsymbol{y}_{e} \cdot \nabla \psi^{*}-\gamma \boldsymbol{\varphi}^{*} \cdot \boldsymbol{e}_{d} & =\lambda \psi^{*} & & \text { in } \Omega \\
\operatorname{div} \boldsymbol{\varphi}^{*} & =0 & & \text { in } \Omega \\
\left\{\varphi^{*(1)}, \ldots, \varphi^{*(d-1)}\right\}=0, \psi^{*} & =0 & & \text { on } \omega
\end{aligned}\right.
$$

with over-determination in (B.5d) Then

$$
\varphi^{*}=0, \psi^{*}=0, \pi=\text { const in } \Omega
$$

Proof of $D^{*} \mathcal{B}^{*} f=-\left.\kappa \frac{\partial f}{\partial \nu}\right|_{\Gamma}, f \in \mathcal{D}\left(\mathcal{B}_{q}^{*}\right)$, in (4.18) essentially by Green's formula.

1. Let $1<q<\infty$ and define

$$
B_{o, q} h=\boldsymbol{y}_{e} \cdot h, L^{q}(\Omega) \supset \mathcal{D}\left(B_{o, q}\right)=W_{0}^{1, q}(\Omega) \longrightarrow L^{q}(\Omega)
$$

Then

$$
B_{o, q}^{*} h=-\boldsymbol{y}_{e} \cdot f, L^{q^{\prime}}(\Omega) \supset \mathcal{D}\left(B_{o, q}^{*}\right)=W_{0}^{1, q^{\prime}}(\Omega) \longrightarrow L^{q^{\prime}}(\Omega)
$$

In fact recalling $\left.\boldsymbol{y}_{e}\right|_{\Gamma} \equiv 0$ and $\operatorname{div} \boldsymbol{y}_{e} \equiv 0$ in $\Omega$ from (1.2c-d $)$, we compute in the duality pairing $L^{q}(\Omega), L^{q^{\prime}}(\Omega)$, with $h \in \mathcal{D}\left(B_{o, q}\right), f \in \mathcal{D}\left(B_{o, q}^{*}\right)$ by (B.7):

$$
\begin{aligned}
\left\langle B_{o, q} h, f\right\rangle & =\int_{\Omega} f \boldsymbol{y}_{e} \cdot \nabla h d \Omega=\int_{\Gamma} h f \boldsymbol{y}_{e} \nabla d \Gamma-\int_{\Omega} h \operatorname{div}\left(f \boldsymbol{y}_{e}\right) d \Omega \\
& =-\int_{\Omega} h \boldsymbol{y}_{e} \cdot \nabla f d \Omega=\left\langle h, B_{o, q}^{*} f\right\rangle_{\Omega}
\end{aligned}
$$

2. We return to the operator $\mathcal{B}_{q}$ in (1.18), with $h \in \mathcal{D}\left(\mathcal{B}_{q}\right)$

$$
\mathcal{B}_{q} h=-\kappa \Delta h+B_{o, q} h, \text { so that } \mathcal{B}_{q}^{*} h=-\kappa \Delta f+B_{o, q}^{*} f, f \in \mathcal{D}\left(\mathcal{B}_{q}^{*}\right)
$$

We shall show that

$$
D^{*} \mathcal{B}^{*} f=-\left.\kappa \frac{\partial f}{\partial \nu}\right|_{\Gamma}, f \in \mathcal{D}\left(\mathcal{B}_{q}^{*}\right)=W^{2, q^{\prime}}(\Omega) \cap W_{0}^{1, q^{\prime}}(\Omega)
$$

In fact, by (B.8) and (B.10) we compute with $v \in L^{2}(\Gamma)$ recalling the definition of $D$ in (1.23a)

$$
\begin{aligned}
\left\langle D^{*} \mathcal{B}_{q}^{*} f, v\right\rangle_{\Gamma} & =\left\langle\mathcal{B}_{q}^{*} f, D v\right\rangle_{\Omega}=\langle\Delta f,-\kappa D v\rangle_{\Omega}+\left\langle f, B_{o, q}^{*} D v\right\rangle_{\Omega} \\
& =\left\langle f,\left(-\kappa \Delta-\boldsymbol{y}_{e} \cdot \nabla\right) D v\right\rangle_{\Omega}+\int_{\Gamma} \frac{\partial f}{\partial \nu}(-\kappa D v) d \Gamma+\int_{\Gamma} f\left(\kappa \frac{\partial D \gamma}{\partial \nu}\right) d \Gamma \\
& =\left\langle-\kappa \frac{\partial f}{\partial \nu}, v\right\rangle_{\Gamma}, \quad \text { for all } v \in L^{q}(\Gamma) .
\end{aligned}
$$

and (B.11) is established. 


\section{Validation of the Kalman controllability conditions (4.31) with fluid vectors $\left\{\boldsymbol{u}_{1}, \ldots, \boldsymbol{u}_{\ell_{i}}\right\}, \ell_{i} \leq K, i=1, \ldots, M$ having only $(d-1)$ components.}

For the sake of the clarity, we shall consider separately the cases $d=2$ and $d=3$.

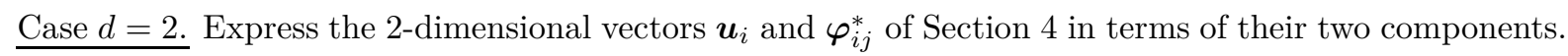

$$
\boldsymbol{u}_{i}=\left[u_{i}^{(1)}, u_{i}^{(2)}\right], \boldsymbol{\varphi}_{i j}^{*}=\left[\varphi_{i j}^{*(1)}, \varphi_{i j}^{*(2)}\right], i=1, \ldots, M, j=1, \ldots, \ell_{i}
$$

Here we shall recall the matrix $\boldsymbol{W}_{i}$ in (4.24) but we shall replace the matrix $U_{i}$ in (4.25) with the following matrix $U_{i}^{(2)}$ :

$$
U_{i}^{(2)}=\left[\begin{array}{ccc}
\left\langle u_{1}^{(2)}, \varphi_{i 1}^{*(2)}\right\rangle_{\omega} & \cdots & \left\langle u_{\ell_{i}}^{(2)}, \varphi_{i 1}^{*(2)}\right\rangle_{\omega} \\
\left\langle u_{2}^{(2)}, \varphi_{i 2}^{*(2)}\right\rangle_{\omega} & \cdots & \left\langle u_{\ell_{i}}^{(2)}, \varphi_{i 2}^{*(2)}\right\rangle_{\omega} \\
\vdots & & \vdots \\
\left\langle u_{1}^{(2)}, \varphi_{i \ell_{i}}^{*(2)}\right\rangle_{\omega} & \cdots & \left\langle u_{\ell_{i}}^{(2)}, \varphi_{i \ell_{i}}^{*(2)}\right\rangle_{\omega}
\end{array}\right]: \ell_{i} \times K
$$

where now the duality pairing $\langle,\rangle_{\omega}$ involves two scalar functions. Next, for $d=2$, we shall establish the Kalman algebraic rank condition for the finite dimensional, unstable, feedback problem (4.23), by employing not the full strength of the 2-dimensional vectors $\boldsymbol{u}_{i}$ and $\boldsymbol{\varphi}_{i j}^{*}$ as in (4.31) involving the matrix $U_{i}$ in (4.25), but instead replacing $U_{i}$ with $U_{i}^{(2)}$. This way, only the second (scalar) components $u_{i}^{(2)}$ and $\varphi_{i j}^{*(2)}$ of the 2-dimensional vectors $\boldsymbol{u}_{i}$ and $\varphi_{i j}^{*}$ in (C.1) are needed. Recall that we are dealing with a pair $\{\omega, \widetilde{\Gamma}\}$ as in Fig 1. The counterpart of Theorem 4.1 is now

Theorem C.1. Let $d=2$. It is possible to select boundary vectors $f_{1}, \ldots, f_{K}$ in $\mathcal{F} \subset W^{2-{ }^{1 / q}, q}(\widetilde{\Gamma})$ with support on $\widetilde{\Gamma}$, and scalar second components $\left\{u_{1}^{(2)}, \ldots, u_{\ell_{i}}^{(2)}\right\}$ as in (C.1), such that

$$
\operatorname{rank}\left[W_{i}, U_{i}^{(2)}\right]=\ell_{i}, i, \ldots, M .
$$

Proof. We shall appropriately modify the proof of Theorem 4.1. As in this proof, the crux is to establish that we cannot have simultaneously

$$
\partial_{\nu} \psi_{i \ell_{i}}^{*}=\sum_{j=1}^{\ell_{i}-1} \alpha_{j} \partial_{\nu} \psi_{i j}^{*} \text { in } L^{q}(\widetilde{\Gamma}) \text { and } \varphi_{i \ell_{i}}^{*(2)}=\sum_{j=1}^{\ell_{i}-1} \alpha_{j} \varphi_{i j}^{*(2)} \text { in } L_{\sigma}^{q}(\omega)
$$

with the same constant $\alpha_{1}, \ldots, \alpha_{\ell_{i}-1}$ in both expression.

Claim: Statement (C.4) is false. By contradiction, suppose that both linear combinations in (C.4) hold true. Next, as in (4.34) define the $(d+1)$-vector $\boldsymbol{\Phi}^{*} \equiv\left\{\boldsymbol{\varphi}^{*}, \psi^{*}\right\}$ by

$$
\boldsymbol{\Phi}^{*} \equiv\left[\begin{array}{c}
\boldsymbol{\varphi}^{*} \\
\psi^{*}
\end{array}\right]=\sum_{j=1}^{\ell_{i}-1}\left[\begin{array}{c}
\alpha_{j} \boldsymbol{\varphi}_{i j}^{*} \\
\alpha_{j} \psi_{i j}^{*}
\end{array}\right]-\left[\begin{array}{c}
\boldsymbol{\varphi}_{i \ell_{i}}^{*} \\
\psi_{i \ell_{i}}^{*}
\end{array}\right]=\sum_{j=1}^{\ell_{i}-1} \alpha_{j} \boldsymbol{\Phi}_{i j}^{*}-\boldsymbol{\Phi}_{i \ell_{i}}^{*}, i=1, \ldots, M ; q \geq 2,
$$

in $\boldsymbol{W}_{\sigma}^{q}(\Omega) \equiv \boldsymbol{L}_{\sigma}^{q}(\Omega) \times L^{q}(\Omega)$, with $\boldsymbol{\Phi}_{i j}^{*} \equiv\left\{\boldsymbol{\varphi}_{i j}^{*}, \psi_{i j}^{*}\right\}$ eigenvector of $\mathbb{A}_{q, N}^{*}$ or $\mathbb{A}_{q}^{*}$, as in (4.3). Then, in view of (C.4), we now obtain

$$
\varphi^{*(2)} \equiv 0 \text { in } \omega,\left.\quad \partial_{\nu} \psi^{*}\right|_{\Gamma} \equiv 0 .
$$


(in place of (4.35)). As in the proof of Theorem 4.1, since the $\Phi_{i j}^{*}$ are eigenvectors of $\mathbb{A}_{q, N}^{*}$, or $\mathbb{A}_{q}^{*}$, so is the vector $\boldsymbol{\Phi}^{*} \equiv\left\{\boldsymbol{\varphi}^{*}, \psi^{*}\right\}$ defined in (C.5). Thus $\boldsymbol{\Phi}^{*} \equiv\left\{\boldsymbol{\varphi}^{*}, \psi^{*}\right\}$ satisfies the PDE version (4.36a-d) of the eigenproblem for the dual $\mathbb{A}_{q}^{*}$, which is now augmented with the over-determined conditions in (C.6). The fact that now $\left.\varphi^{*}\right|_{\Gamma}=0$ by (4.36d) on the entire boundary $\Gamma$, permits a-fortiori the argument of [89, Theorems 6, 7] to hold true. Namely, invoke $\varphi^{*(2)} \equiv 0$ in $\omega$ in (C.6) to obtain, as in the proof of Theorem 4.1, the over-determined problem (4.37) for $\psi^{*}$ on $\omega$, with the conclusion that

$$
\psi^{*} \equiv 0 \text { in } \omega, \text { as in (4.38) }
$$

Next, the divergence condition (4.36c) yields in view of (C.6): $\operatorname{div} \varphi^{*}=\varphi_{x_{1}}^{*(1)}+\varphi_{x_{2}}^{*(2)}=\varphi_{x_{1}}^{*(1)}=0$ in $\omega$. Hence $\varphi^{*(1)}\left(x_{1}, x_{2}\right) \equiv c\left(x_{2}\right)$ in $\omega$, where $c\left(x_{2}\right)$ is a function constant w.r.t. $x_{1}$ and depending only on $x_{2}$ in $\omega$. Next, let $P=\left\{x_{1}(P), x_{2}(P)\right\}$ be an arbitrary point of $\omega$. Consider the line $\ell$ passing through the point $P$ and parallel to the $x_{1}$-axis. On such a line $\ell$, the value $\varphi^{*(1)}\left(x_{1}, x_{2}(P)\right) \equiv c_{2}\left(x_{2}(P)\right)$ is constant w.r.t. $x_{1}$, as long as $\ell$ intersect $\omega$. By definition of the small set $\omega$ supported by $\widetilde{\Gamma}$, there is a non-empty open subset $\widetilde{\omega} \subset \omega$, where this happens: for all points $P$ in $\widetilde{\omega}$, the line $\ell$ remains in $\widetilde{\omega}$ and hits the boundary $\widetilde{\Gamma}$, where condition (4.36d) applies for $\left.\varphi^{*(1)}\right|_{\Gamma}=0$. Thus $\varphi^{*(1)} \equiv 0$ in $\widetilde{\omega} \subset \omega$. Recalling (C.6), we finally have

$$
\varphi^{*}=\left\{\varphi^{*(1)}, \varphi^{*(2)}\right\} \equiv 0 \text { in } \widetilde{\omega} \subset \omega, \text { along with } \psi^{*} \equiv 0 \text { in } \widetilde{\omega} \text { by (C.7). }
$$

We can then apply $[89$, Theorem 5 for $\widetilde{\omega}]$ and conclude that

$$
\varphi^{*}=\left\{\varphi^{*(1)}, \varphi^{*(2)}\right\} \equiv 0 \text { in } \Omega, \psi^{*} \equiv 0 \text { in } \Omega, p \equiv \text { const in } \Omega .
$$

The rest of the proof proceeds as in Theorem 4.1 following (4.40). Theorem C.1 is established.

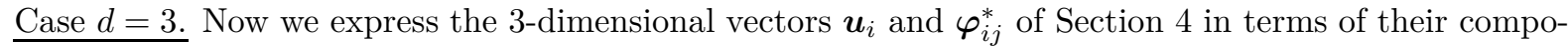
nents as

$$
\boldsymbol{u}_{i}=\left[u_{i}^{(1)}, u_{i}^{(2)}, u_{i}^{(3)}\right], \varphi_{i j}^{*}=\left[\varphi_{i j}^{*(1)}, \varphi_{i j}^{*(2)}, \varphi_{i j}^{*(3)}\right], i=1, \ldots, M, j=1, \ldots, \ell_{i} .
$$

We now distinguish two sub-cases.

Sub-case $\{1,3\}$. Here, we extract only the first and third components, while we omit the second component. Accordingly, we introduce the following 2-dimension vectors $\boldsymbol{u}_{i}^{(1,3)}$ and $\boldsymbol{\varphi}^{*(1,3)}$ and the corresponding matrix $U_{i}^{(1,3)}$

$$
\begin{gathered}
\boldsymbol{u}_{i}^{(1,3)}=\left[u_{i}^{(1)}, u_{i}^{(3)}\right], \varphi_{i j}^{*(1,3)}=\left[\varphi_{i j}^{*(1)}, \varphi_{i j}^{*(3)}\right] \\
U_{i}^{(1,3)}=\left[\begin{array}{lll}
\left\langle\left[\begin{array}{c}
u_{1}^{(1)} \\
u_{1}^{(3)}
\end{array}\right],\left[\begin{array}{l}
\varphi_{i 1}^{*(1)} \\
\varphi_{i 1}^{*(3)}
\end{array}\right]\right\rangle_{\omega} & \ldots & \left\langle\left[\begin{array}{l}
u_{\ell_{i}}^{(1)} \\
u_{\ell_{i}}^{(3)}
\end{array}\right],\left[\begin{array}{c}
\varphi_{i 1}^{*(1)} \\
\varphi_{i 1}^{*(3)}
\end{array}\right]\right\rangle_{\omega} \\
\left\langle\left[\begin{array}{c}
u_{1}^{(1)} \\
u_{1}^{(3)}
\end{array}\right],\left[\begin{array}{l}
\varphi_{i \ell_{i}}^{*(1)} \\
\varphi_{i \ell_{i}}^{*(3)}
\end{array}\right]\right\rangle_{\omega} & \ldots & \left\langle\left[\begin{array}{c}
u_{\ell_{i}}^{(1)} \\
u_{\ell_{i}}^{(3)}
\end{array}\right],\left[\begin{array}{c}
\varphi_{i \ell_{i}}^{*(1)} \\
\varphi_{i \ell_{i}}^{*(3)}
\end{array}\right]\right\rangle_{\omega}
\end{array}\right]
\end{gathered}
$$

Sub-case $\{2,3\}$. Here, we extract only the second and third components, while we omit the first component. Accordingly, we introduce the following 2-dimension vectors $\boldsymbol{u}_{i}^{(2,3)}$ and $\boldsymbol{\varphi}^{*(2,3)}$ and the corresponding matrix $U_{i}^{(2,3)}$

$$
\boldsymbol{u}_{i}^{(2,3)}=\left[u_{i}^{(2)}, u_{i}^{(3)}\right], \varphi_{i j}^{*(2,3)}=\left[\varphi_{i j}^{*(2)}, \varphi_{i j}^{*(3)}\right]
$$




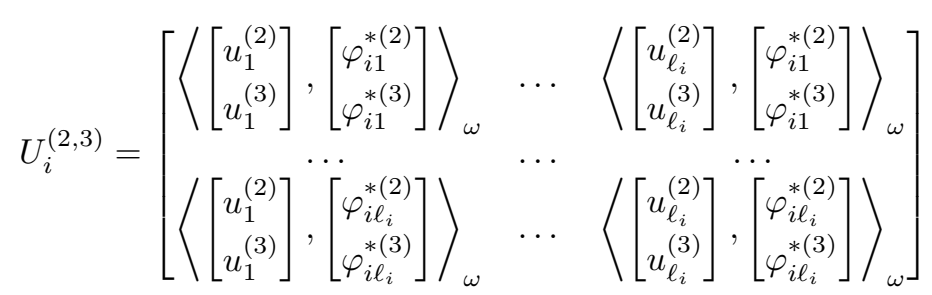

Next, for $d=3$, we shall establish the Kalman algebraic rank condition for the finite dimensional, unstable, feedback problem (4.23), by replacing the matrix $U_{i}$ in (4.25), with either the matrix $U_{i}^{(1,3)}$ in (C.11) (Sub-case $\{1,3\}$ ); or else with the matrix $U_{i}^{2,3}$ in (C.12) (Sub-case $\{2,3\}$ ). This way, the third (scalar) components $u_{i}^{(3)}$ and $\varphi_{i j}^{*(3)}$ of the 3 -dimensional vectors $\boldsymbol{u}_{i}$ and $\boldsymbol{\varphi}_{i j}^{*}$ in (C.10) are needed in both sub-cases. The counterpart of Theorem 4.1 (or Theorem C.1) is now

Theorem C.2. Let $d=3$. It is possible to select boundary vectors $f_{1}, \ldots, f_{K}$ in $\mathcal{F} \subset W^{2-1 / q, q}(\widetilde{\Gamma})$ with support on $\widetilde{\Gamma}$, and

(i) either 2-dimensional vectors $\boldsymbol{u}_{i}^{(1,3)}=\left\{u_{i}^{(1)}, u_{i}^{(3)}\right\}$ as in (C.11)

(ii) or else 2-dimensional vectors $\boldsymbol{u}_{i}^{(2,3)}=\left\{u_{i}^{(2)}, u_{i}^{(3)}\right\}$ as in (‥12),

such that

$$
\operatorname{rank}\left[W_{i}, U_{i}^{(1,3)}\right]=\ell_{i}, i, \ldots, M, \text { in case }(i)
$$

or

$$
\operatorname{rank}\left[W_{i}, U_{i}^{(2,3)}\right]=\ell_{i}, i, \ldots, M, \text { in case }(i i)
$$

respectively.

Proof. We shall only give a proof in the sub-case $\{1,3\}$, as the proof of the sub-case $\{2,3\}$ is the same mutatis mutandis. As in the proof of Theorem 4.1 (or Theorem C.1), the crux is to establish that we cannot have simultaneously

$$
\partial_{\nu} \psi_{i \ell_{i}}^{*}=\sum_{j=1}^{\ell_{i}-1} \alpha_{j} \partial_{\nu} \psi_{i j}^{*} \text { in } L^{q}(\widetilde{\Gamma}) \quad \text { and } \quad \varphi_{i \ell_{i}}^{*(1,3)}=\sum_{j=1}^{\ell_{i}-1} \alpha_{j} \varphi_{i j}^{*(1,3)} \text { in } \boldsymbol{L}_{\sigma}^{q}(\omega)
$$

with the same constants $\alpha, \ldots, \alpha_{\ell_{i}-1}$ in both expressions.

Claim: Statement (C.15) is false. By contradiction, suppose that both linear combinations in (C.15) hold true. Next, define the $(d+1)$-vector $\boldsymbol{\Phi}^{*} \equiv\left\{\boldsymbol{\varphi}^{*}, \psi^{*}\right\}$ as in (4.34) (or (C.5)). Then, in view of (C.15), we now obtain

$$
\varphi^{*(1,3)} \equiv\left\{\varphi^{*(1)}, \varphi^{*(3)}\right\} \equiv 0 \text { in } \omega,\left.\quad \partial_{\nu} \psi^{*}\right|_{\widetilde{\Gamma}} \equiv 0 .
$$

(in place of (4.35) or (C.6)). As in Theorem 4.1 (or Theorem C.1) the $\boldsymbol{\Phi}^{*} \equiv\left\{\boldsymbol{\varphi}^{*}, \psi^{*}\right\}$ is an eigenvector of $\mathbb{A}_{q}^{*}$ and thus satisfies the corresponding PDE-version - that is problem (4.36a-d), augmented this time with the over-determined conditions in (C.16). Next, invoke $\varphi^{*(3)} \equiv 0$ in $\omega$ from (C.16) to obtain, as this the proof of Theorem 4.1, the over-determined problem (4.37) for $\psi^{*}$ in $\omega$, with the conclusion that

$$
\psi^{*} \equiv 0 \text { in } \omega,
$$

as in (4.36) (or (C.7)). Next, the divergence condition (4.36c) yields in the view of (C.16): div $\varphi^{*}=$ $\varphi_{x_{1}}^{*(1)}+\varphi_{x_{2}}^{*(2)}+\varphi_{x_{3}}^{*(3)}=\varphi_{x_{2}}^{*(2)}=0$ in $\omega$. Hence $\varphi^{*(2)}\left(x_{1}, x_{2}, x_{3}\right) \equiv c\left(x_{1}, x_{3}\right)$ in $\omega$, where $c\left(x_{1}, x_{3}\right)$ is a function constant w.r.t. $x_{2}$ and depending only on $x_{1}$ and $x_{3}$ in $\omega$. Next, let $P=\left\{x_{1}(P), x_{2}(P), x_{3}(P)\right\}$ be an arbitrary point of $\omega$. Consider the plane $\pi_{P}$ passing through the point $P$ and parallel to the $\left\{x_{1}, x_{3}\right\}$-coordinate plane. As the point $\left\{x_{1}, x_{2}(P), x_{3}\right\}$ of $\omega$ runs over the plane $\pi_{P}$, the value 
$\varphi^{*(2)}\left(x_{1}, x_{2}(P), x_{3}\right)=c\left(x_{1}, x_{3}\right)$ is independent of $x_{2}(P)$, as long as such plane $\pi_{P}$ intersects $\omega$. By definition of the small $\omega$ supported by $\widetilde{\Gamma}$, there is a non-empty open subset $\widetilde{\omega} \subset \omega$, where this happens: for all points $P$ in $\widetilde{\omega}$, the plane $\pi_{P}$ remains in $\widetilde{\omega}$ and hits the boundary $\widetilde{\Gamma}$, where condition (4.36d) applies for $\left.\varphi^{*(2)}\right|_{\Gamma}=0$. Thus $\varphi^{*(2)} \equiv 0$ in $\widetilde{\omega} \subset \omega$. Recalling (C.16) we conclude that

$$
\varphi^{*}=\left\{\varphi^{*(1)}, \varphi^{*(2)}, \varphi^{*(3)}\right\} \equiv 0 \text { in } \widetilde{\omega} \subset \omega, \text { along with } \psi^{*} \equiv 0 \text { in } \widetilde{\omega} \text { by }(\text { C.17). }
$$

We can then apply [89, Theorem 5 for $\widetilde{\omega}]$ and conclude that

$$
\varphi^{*}=\left\{\varphi^{*(1)}, \varphi^{*(2)}, \varphi^{*(3)}\right\} \equiv 0 \text { in } \Omega, \psi^{*} \equiv 0 \text { in } \Omega, p \equiv \text { const in } \Omega .
$$

The rest of the proof proceeds as in Theorem 4.1 following (4.40). Theorem C.2 is established.

The authors wish to thank the referee for much appreciated comments and suggestions.

\section{References}

[1] H. Amann, Linear and Quasilinear Parabolic Problems: Volume I: Abstract Linear Theory, Birkhäuser, 1995, pp 338.

[2] H. Amann, On the Strong Solvability of the Navier-Stokes Equations, J. Math. Fluid Mech. 2 , 2000, pp 16-98.

[3] P. Acevedo Tapia, $L^{p_{-}}$Theory for the Boussinesq system, Ph.D theis, Universidad de Chille,Faculatad de Ciencias Fisicas y Mathematicas, Departamento de Ingeniearia Mathematica, Santiago de Chille, 2015

[4] P. Acevedo, C. Amrouche, C. Conca, Boussinesq system with non-homogeneous boundary conditions, Applied Mathematics Letter, 53(2016) 39-44

[5] P. Acervedo, C. Amrouche, C. Conca, $L^{p}$ theory for Boussinesq system with Dirichlet boundary conditions, Applicable Analysis(2019), V.98 N.1-2, 272-294

[6] A. V. Balakrishnan, Applied Functional Analysis, Springer Verlag, Applications of mathematics Series, 2nd Edit 1981, pp 369.

[7] V.Barbu, Stabilization of Navier-Stokes flows, Springer 2011.

[8] G.Basile, G.Marro Controlled and Conditioned Invariant in Linear System Theory. Prentice Hall 1992.

[9] V. Barbu, R. Triggiani, Internal Stabilization of Navier-Stokes Equations with Finite-Dimensional Controllers, Indiana University Mathematics, 2004, pp 1443-1494.

[10] V. Barbu, I. Lasiecka, R. Triggiani, Tangential Boundary Stabilization of Navier-Stokes Equations, Memoires of American Math Society, 2006, pp 128.

[11] V. Barbu, I. Lasiecka, R. Triggiani, Abstract settings fr tangential boundary stabilization of Naiver Stokes equations by high-and low-gain feedback controllers, Nonlinear Analysis 64(12)(June 2006), 2705-2746

[12] V. Barbu, I. Lasiecka, R. Triggiani, Local exponential stabilization strategies of the Navier Stokes equations, $d=2,3$, via feedback stabilization of its linearization, Optimal of Coupled Systems of Partial Differential Equations, Oberwolfach, Birkhäuser ISNM 155(2007), 13-46.

[13] A. L. Bukhgeim, Carleman estimates for Volterra operators and uniquness of inverse problems in non-classical problem of mathematical physics, Computer Center, Siberian Branch of USSR Academy of Sciences, Novosibirsk, pp 56-54 (in Russian). 
[14] A. L. Bukhgeim, M. V. Klibanov, Uniqueness in the large of a class of multidimensional inverse problems, Sov. Math. Dokl. 17, 244-247 (1981).

[15] J. A. Burns, W. Hu, Approximation methods for boundary control of the Boussinesq equations, 52nd IEEE Conference on Decision and Control. IEEE, 2013, p 454-459.

[16] J. A. Burns, X. He, W. Hu, Feedback stabilization of a thermal fluid system with mixed boundary control, Computers and Mathematics with Applications, 71(11), 2170-2191. https://doi.org/10.1016/j.camwa.2016.01.011, (2016).

[17] T. Carleman, Sur un probleme d'unicite pour les systémes d'équations aux dérivées partielles \& deux variables independentes Ark. Mat 26 (1939) 1-9.

[18] C. T. Chen, Linear Systems Theory and Design, Oxford University Press, 1984, pp 334.

[19] P. Constantin, C. Foias, Navier-Stokes Equations, Chicago Lectures in Mathematics 1st Edition, 1980, pp 190.

[20] J. M. Coron, S. Guerrero, Null controllability of the N dimensional Stokes system with N-1 scalar controls, J.Differential Equations 246(2009)2908-2921.

[21] J. M. Coron, P. Lissy, Local null controllability of the three-dimensional Navier-Stokes system with a distributed control having two vanishing components. Inv. Math. ,3 (198) (2014), 833-880.

[22] L. De Simon, Un'applicazione della teoria degli integrali singolari allo studio delle equazioni differenziali lineari astratte del primo ordine, Rendiconti del Seminario Matematico della Universita di Padova 34 (1964), 205-223.

[23] G. Dore, Maximal regularity in $L^{p}$ spaces for an abstract Cauchy problem, Advances in Differential Equations, 2000.

[24] L. Escauriaza, G. Seregin, V. Šverák, $L_{3, \infty}$-Solutions of Navier-Stokes Equations and Backward Uniqueness, Mathematical subject classification (Amer. Math. Soc.): 35K, 76D, 1991.

[25] E. Fabes, O. Mendez, M. Mitrea, Boundary Layers of Sobolev-Besov Spaces and Poisson's Equations for the Laplacian for the Lipschitz Domains, J. Func. Anal 159(2):, 1998, pp 323-368.

[26] E. Fernandez Cara, M. Santos, D. Souza, Boundary controllability of incompressible Euler fluids with Boussinesq heat effects, Math Control Signals (2016), 28: 7.

[27] C. Foias, R. Temam, Determination of the Solution of the Navier-Stokes Equations by a Set of Nodal Volumes, Mathematics of Computation, Vol 43, N 167, 1984, pp 117-133.

[28] A. V. Fursikov, Stabilization for the 3D Navier-Stokes system by feedback boundary control, Discrete and Continuous Dynamical Systems 10.1/2 (2004): 289-314.

[29] G. P. Galdi, An Introduction to the Mathematical Theory of the Navier-Stokes Equations, Volume - I: Nonlinear Steady Problems. Springer-Verlag New York, 1994, pp 465.

[30] G. P. Galdi, An Introduction to the Mathematical Theory of the Navier-Stokes Equations, Volume - II: Linearized Steady Problems. Springer-Verlag New York, 1994, pp 323.

[31] G. P. Galdi, An Introduction to the Mathematical Theory of the Navier-Stokes Equations, SpringerVerlag New York, 2011.

[32] M. Geissert, K. Götze, M. Hieber, $L_{p}$-Theory for Strong Solutions to Fluid-Rigid Body Interaction in Newtonian and Generalized Newtonian Fluids. Transaction of American Math Society, 2013, pp 1393-1439.

[33] Y. Giga, Analyticity of the semigroup generated by the Stokes operator in $L_{r}$ spaces, Math.Z.178(1981), n 3, pp 279-329.

[34] Y. Giga, Domains of fractional powers of the Stokes operator in $L_{r}$ spaces, Arch. Rational Mech. Anal. 89(1985), n 3, pp 251-265. 
[35] S. Guerrero, Local exact controllability to the trajectories of the Boussinesq system, Annales de l'Institut Henri Poincaré (C) Non Linear Analysis, Vol 23(1), Jan.-Feb. 2006, pp 29-61.

[36] L. Hörmander, The analysis of linear partial differential operators III, Springer, 1985.

[37] M. Hieber, J. Saal, The Stokes Equation in the $L^{p}$-setting: Well Posedness and Regularity Properties Handbook of Mathematical Analysis in Mechanics of Viscous Fluids, Springer, Cham,, 2016, pp 1-88.

[38] V. Isakov, Inverse Problems for Partial Differential Equations, Springer Verlag, Berlin.

[39] H. Jia, V. Šverák, Minimal $L^{3}$-initial data for potential Navier-Stokes singularities, SIAM J. Math. Anal. 45 (2013), no. 3, 1448-1459.

[40] T. Kato, Perturbation Theory of Linear Operators, Springer-Verlag, 1966, pp 623.

[41] S. Kesavan, Topics in Functional Analysis and Applications, New Age International Publisher, 1989, pp 267.

[42] V. A. Khoa, M. V. Klibanov, L. H. Nguyen, Convexification for a Three-Dimensional Inverse Scattering Problem with the Moving Point Source, SIAM Journal on Imaging Sciences, Vol-13, No 2, pp 871-904, 2020.

[43] Convexification and experimental data for a 3D inverse scattering problem with the moving point source V. A. Khoa, G. W. Bidney, M. V. Klibanov, L. H. Nguyen, L. H. Nguyen, A. J. Sullivan, V. N. Astratov. Inverse Problems, 36 (2020) 085007 (34pp), https://doi.org/10.1088/1361-6420/ab95aa.

[44] H. Kim, The existence and uniqueness of very weak solutions of the stationary Boussinesq system, Nonlinear Analysis: Theory, Methods $\& 3$ Applications 75.1 (2012): 317-330.

[45] M. V. Klibanov, Uniqueness of solutions in the large of some multidimensional inverse problems, Non-classical problems of mathematical physics, Computer science, Siberian Branch of USSR Academy of Sciences, Novosibirsk, 1981, pp 101-114 (in Russian).

[46] M. V. Klibanov, On a class of inverse problems, Sov. Math. Dokl. 26, 1982, pp 248-250.

[47] M. V. Klibanov, Inverse problems in the large and Carleman bounds, Diff. Eqts, 20 (1984), pp 755-760.

[48] M. V. Klibanov Carleman estimates for global uniqueness, stability and numerical methods for coefficient inverse problems, J. Inverse Ill-Posed Probl. 21 (2013), pp 477 - 560.

[49] M. Kazemi, M. V. Klibanov, Stability estimates for ill-posed Cauchy problems invoking hyperbolic equations and inequalities, Applicable analysis 50, (1993), 93-102.

[50] M. V. Klibanov, A. A. Timonov, Carleman estimates for Coefficient Inverse Problems and Numerical Applications, Inverse and Ill-posed problem series, Urecht-Boston 2004, 282pp.

[51] V. Kormonik, Exact Controllability and Stabilization - The multiplier method, 1994.

[52] I. Kukavica and A. Tuffaha, Regularity of solutions to a free boundary problem of fluid-structure interaction, Indiana University Math J., 61 (2012), N 5, 1817-1859

[53] P. C. Kunstmann, L. Weis Perturbation theorems for maximal $L^{p}$-regularity, Annali della Scuola Normale Superiore di Pisa, Classe Scienze, Serie 4, volume 30 (2001) 415-435

[54] P. C. Kunstmann, L. Weis Maximal $L^{p}$-regularity for Parabolic Equations, Fourier Multiplier Theorems and $H^{\infty}$-functional Calculus Functional Analytic Methods for Evolution Equations, Lecture Notes in Mathematics, vol 1855. Springer, Berlin, Heidelberg pp 65-311.

[55] O. A. Ladyzhenskaya, The Mathematical Theory of Viscous Incompressible Flow, Gordon and Breach, New York English transl., $2^{\text {nd }}$ Edition, 1969.

[56] I. Lasiecka, R. Triggiani, Uniform Stabilization with Arbitrary Decay Rates of the Oseen Equation by Finite-Dimensional Tangential Localized Interior and Boundary Controls, Semigroups of Operators -Theory and Applications, 2015, pp 125-154. 
[57] I. Lasiecka, R. Triggiani, Stabilization to an Equilibrium of the Navier-Stokes Equations with Tangential Action of Feedback Controllers, Nonlinear Analysis, 2015, pp 424-446.

[58] I. Lasiecka, R. Triggiani, Control Theory of Partial Differential Equations: Continuous and Approximation Theories, Vol 1 Abstract Parabolic Equations (680pp), Encyclopedia of Mathematics and its Applications Series, Cambridge University Press, January 2000.

[59] S. Liu, R. Triggiani, Boundary Control and Boundary Inverse Theory for Non-Homogeneous Second-Order Hyperbolic Equation, A common Carleman Estimate Approach, HCDTE Lecture Notes, Pert I.

[60] I. Lasiecka, B. Priyasad, R. Triggiani, Uniform Stabilization of Navier-Stokes Equations in Critical $L^{q}$-Based Sobolev and Besov Spaces by Finite Dimensional Interior Localized Feedback Controls. Appl. Math Optim. (2019). https://doi.org/10.1007/s00245-019-09607-9.

[61] I. Lasiecka, B. Priyasad, R. Triggiani, Uniform stabilization of Boussinesq systems in critical $\boldsymbol{L}^{q}$ based Sobolev and Besov spaces by finite dimensional interior localized feedback controls, Discrete \& Continuous Dynamical Systems - B,25,10,4071,4117,2020-6-15.

[62] I. Lasiecka, B. Priyasad, R. Triggiani, Uniform stabilization of $3 D$ Navier-Stokes Equations with finite dimensional tangential boundary, localized feedback controllers. submitted.

[63] M. M. Lavrentev, V. G. Romanov, S. P. Shishataskii, Ill-posed problems in Mathematical Physics and Analysis, Amer. Math. Soc. Vol 64 (1986).

[64] A. I. Lefter, On the Feedback Stabilization of Boussinesq Equations ANALELE STIINTIFICE ALE UNIVERSIT ATII "AL.I. CUZA" DIN IASI (SN) MATEMATICA, Tomul LVII, 2011, f.2.

[65] J. L. Lions, Quelques Methodes de Resolutions des Problemes aux Limites Non Lineaire, Dunod, Paris, 1969.

[66] S. A. Lorca, J. L. Boldrini, Stationary Solution for Generalized Boussinesq Models, Journal of Differential Equations 124, 1996, 389-406.

[67] V. Maslenniskova, M. Bogovskii, Elliptic Boundary Values in Unbounded Domains with Non Compact and Non Smooth Boundaries Rend. Sem. Mat. Fis. Milano, 56, 1986, 125-138.

[68] A. Pazy, Semigroups of Linear Operators and Applications to Partial Differential Equations, Springer-Verlag, 1983.

[69] J. Prüss, G. Simonett, Moving Interfaces and Quasilinear Parabolic Evolution Equations Birkhäuser Basel, Monographs in Mathematics 105, 2016. 609pp.

[70] J. Prüss and R.Schaubelt, Solvability and maximal regularity of parabolic equations with coefficients continuous in time, JMAA 256 (2001), 405-430.

[71] M. Ramaswamy, J-P. Raymond, A. Roy, Boundary Feedback Stabilization of the Boussinesq system with mixed boundary conditions, Journal of Differential Equations, Elsevier, In press. ffhal$01970455 f$.

[72] W. Rusin, V. Sverak, Minimal initial data for potential Navier-Stokes singularities, https://arxiv.org/abs/0911.0500.

[73] J. Saal, Maximal regularity for the Stokes system on non-cylindrical space-time domains, J. Math. Soc. Japan 58 (2006), no. 3, 617-641.

[74] M. Santos da Rocha, Marko A. Rojas-Medar, and M. Drina Rojas-Medar, On the existence and uniqueness of the stationary solution to equations of natural convection with boundary data in $L^{2}$, Proceedings: Mathematics, Physical and Engineering Sciences (2003): 609-621.

[75] Y. Shibata, S. Shimizu, On the $L^{p}-L^{q}$ maximal regularity of the Neumann problem for the Stokes equations in a bounded domain, Advanced Studies of Pure Math, 47, 2007, pp 347-362.

[76] V. A. Solonnikov, Estimates of the solutions of a nonstationary linearized system of Navier- Stokes equations, A.M.S. Translations, 75 (1968), 1-116. 
[77] V. A. Solonnikov, Estimates for solutions of non-stationary Navier-Stokes equations, J. Sov. Math., 8, 1977, pp 467-529.

[78] V. A. Solonnikov, On the solvability of boundary and initial-boundary value problems for the Navier-Stokes system in domains with noncompact boundaries. Pacific J. Math. 93 (1981), no. 2, 443-458. https://projecteuclid.org/euclid.pjm/1102736272.

[79] V. A. Solonnikov, On Schauder Estimates for the Evolution Generalized Stokes Problem. Ann. Univ. Ferrara 53, 1996, 137-172.

[80] V. A. Solonnikov, $L^{p}$-Estimates for Solutions to the Initial Boundary-Value Problem for the Generalized Stokes System in a Bounded Domain, J. Math. Sci., Volume 105, Issue 5, pp 2448-2484.

[81] V. A. Solonnikov, Estimates for solutions of non-stationary Navier-Stokes equations, J. Sov. Math., 8, 1977, pp 467-529.

[82] A. E. Taylor, D. Lay, Introduction to Functional Analysis $2^{\text {nd }}$ Edition, Wiley Publication, ISBN-13: 978-0471846468, 1980.

[83] R. Temam, Navier-Stokes Equations, North Holland, 1979, pp 517.

[84] R. Triggiani, On the Stabilizability Problem of Banach Spaces, J. Math. Anal. Appl. 55,1975, pp 303-403.

[85] R. Triggiani, Feedback Stability of Parabolic Equations, Appl. Math. Optimiz. 6, 1975, pp 201-220.

[86] R. Triggiani, Unique Continuation of the boundary over-determined Stokes and Oseen eigenproblems, Discrete and Continuous Dynamical Systems, Series S, Vol 2, N 3, Sept. 2009.

[87] R. Triggiani, Linear independence of boundary traces of eigenfunctions of elliptic and Stokes Operators and applications, invited paper for special issue, Applicationes Mathematicae 35(4) (2008), 481-512, Institute of Mathematics, Polish Academy of Sciences.

[88] R. Triggiani, Unique Continuation from an Arbitrary Interior Subdomain of the Variable-Coefficient Oseen Equation. Nonlinear Analysis, 2009, pp 645-678.

[89] R.Triggiani, X. Wan, Unique continuation properties of over-determined static Boussinesq problems with applications to uniform stabilization of dynamic Boussinesq systems, Appl Math \& Optimiz.

[90] Villamizar-Roa, Elder J., Maria Angeles Rodríguez-Bellido, M. A. Rojas-Medar, The Boussinesq system with mixed nonsmooth boundary data, Comptes Rendus Mathematique 343.3 (2006): 191196.,

[91] W. von Whal, The Equations of Navier-Stokes and Abstract Parabolic Equations. Springer Fachmedien Wiesbaden, Vieweg+Teubner Verlag, 1985.

[92] G. Wang, Stabilization of the Boussinesq equation via internal feedback controls Nonlinear Analysis: Theory, Methods \& Applications 52.2 (2003): 485-506.

[93] L. Weis, A new approach to maximal Lp-regularity. In Evolution Equ. and Appl. Physical Life Sci., volume 215 of Lect. Notes Pure and Applied Math., pages 195-214, New York, 2001. Marcel Dekker.

[94] J. Zabczyk, Mathematical Control Theory: an Introduction, Birkhäuser 1992, 260pp. 